\title{
TAUTOLOGICAL MODULE AND INTERSECTION THEORY ON HILBERT SCHEMES OF NODAL CURVES*
}

\author{
ZIV RAN ${ }^{\dagger}$
}

\begin{abstract}
This paper presents the rudiments of Hilbert-Mumford Intersection (HMI) theory: intersection theory on the relative Hilbert scheme of a family of nodal (or smooth) curves, over a base of arbitrary dimension. We introduce an additive group of geometric cycles, called 'tautological module', generated by diagonal loci, node scrolls, and twists thereof. We determine recursively the intersection action on this group by the discriminant ( big diagonal) divisor and all its powers. We show that this suffices to determine arbitrary polynomials in Chern classes, in particular Chern numbers, for the tautological vector bundles on the Hilbert schemes, which are closely related to enumerative geometry of families of nodal curves.
\end{abstract}

Key words. Hilbert scheme, nodal curves, intersection theory, enumerative geometry.

AMS subject classifications. 14N99, 14H99.

0. Overview. This paper is a contribution to the study of Enumerative Geometry of nodal curves via their subschemes. To illustrate informally, in part, what it is about, we recall a formula from 19th century Algebraic Geometry (see for example [24], p. 377): a nonsingular curve $X$ in complex projective 3 -space admits an expected finite number $n_{4}$ of 4 -secant lines, and that a formula for $n_{4}$ in terms of the degree $d$ and genus $g$ of $X$ can be given: specifically,

$$
24 n_{4}=144-204 d+106 d^{2}-12 d^{2} g-24 d^{3}+2 d^{4}+84 d g-156 g+12 g^{2} .
$$

Standard dimension-counting suggests that given a nice enough $b$-dimensional family of space curves, it will admit a finite number $n_{4+b}$ of $(4+b)$-secant lines, and one can ask for a method to compute $n_{4+b}$ in terms of basic projective characters of the family. The results of this paper provide, inter alia, such a method, as we now proceed to describe.

0.1. Setting. To fix ideas, consider a family of curves given by a flat projective morphism

$$
\pi: X \rightarrow B
$$

over an irreducible base, with fibres

$$
X_{b}=\pi^{-1}(b), b \in B
$$

which are irreducible nonsingular for the generic $b$ and at worst nodal for every $b$. For example, $X$ could be the universal family of automorphism-free curves over the appropriate open subset of $\overline{\mathcal{M}}_{g}$, the moduli space of Deligne-Mumford stable curves. Many questions in the classical projective and enumerative geometry of this family can be naturally phrased, and in a formal sense solved (see for instance [22]), in the context of the relative Hilbert scheme

$$
X_{B}^{[m]}=\operatorname{Hilb}_{m}(X / B) .
$$

*Received February 15, 2012; accepted for publication March 6, 2012. arxiv.org 0905.2229. This article is a revision of portions of arXiv:0803.4512.

$\dagger$ Mathematics Department, UC Riverside, Surge Facility, Big Springs Road, Riverside CA 92521, USA (ziv.ran@ucr.edu). 
This is a universal parameter space for length- $m$ subschemes of $X$ contained in fibres of $\pi$, and carries natural tautological vector bundle $\Lambda_{m}(E)$, associated to any vector bundle $E$ on $X$ (e.g., the relative dualizing sheaf $\omega_{X / B}$ ). One specific example of the enumerative questions which may be considered from this viewpoint is the fundamental class in $\overline{\mathcal{M}}_{\mathrm{g}}$ of the closure of the hyperelliptic locus.

Typically, the geometric questions one wants to consider can be formulated in terms of relative multiple points and multisecants in the family, which can be described in terms of degeneracy loci of bundle maps involving tautological bundles, and the formal solutions involve Chern numbers of those tautological bundles. Thus, turning these formal solutions into meaningful ones requires computing the Chern numbers in question. This problem was stated but, aside from some low-degree cases, left open in [22]. Our main purpose here is to solve this problem in general. More than that, we shall in fact provide a calculus to compute certain images of arbitrary polynomials in the Chern classes of the tautological bundles. In the 'absolute' case $E=\omega_{X / B}$, the computation ultimately reduces these polynomials to polynomials in Mumford's tautological classes [14] on various boundary strata of $B$. The latter are computed via a conjecture of Witten, proved by Kontsevich [9]. It should be mentioned that in the case of the symmetric product (= Hilbert scheme) of a single smooth curve, a complete intersection theory was worked out by Macdonald [13]. On the other hand, the intersection theory of Hilbert schemes of smooth surfaces was investigated deeply by Nakajima, Lehn and others, see [15], [10], [11] and references therein.

0.2. Tautological module: Motivation. Now the framework for our solution is a little different to what is commonly done in similar problems (e.g. Macdonald's set-up). Rather than compute a suitable intersection ring, we will focus primarily on the (intersection) action of the discriminant or big diagonal $\Gamma^{(m)}$ and its powers. The motivation for this approach comes from a result in [22] called the 'Splitting principle'. This says that the total Chern class of pullback of a tautological bundle $\Lambda_{m}(E)$ to the full-flag Hilbert scheme $W^{m}=W^{m}(X / B)$, which maps to the degree- $i$ Hilbert schemes $X_{B}^{[i]}, i \leq m$, can be expressed as a simple decomposable polynomial in the (pullbacks of) $\Gamma^{(i)}, i \leq m$. The recursive analogue of this result, Cor. 3.7 below, says that the pullback of $c\left(\Lambda_{m}(E)\right)$ on the Hilbert scheme $X_{B}^{[m, m-1]}$, parametrizing flags of schemes of lengths $m, m-1$ (which we will call the 'flaglet' Hilbert scheme), is a product of $c\left(\Lambda_{m-1}(E)\right)$ and a polynomial in in discriminants $\Gamma^{(i)}, i \leq m$. It follows that if we assume recursively that we have some reasonable way to express polynomials in $c\left(\Lambda_{m-1}(E)\right)$, say as elements of a 'Tautological module' $T_{R}^{m-1}(X / B)$ and want to do the same for $m$, then we need to determine 2 things:

1. Tautological module in degree $m, T_{R}^{m}(X / B)$, i.e. a group together with an action of $\Gamma^{(m)}$.

2. Transfer calculus, going from $T_{R}^{m-1}(X / B)$ to $T_{R}^{m}(X / B)$ via the flaglet correspondence $X_{B}^{[m, m-1]}$.

Given these, $T_{R}^{m}(X / B)$ would recursively contain all polynomials in $\Gamma^{(i)}, i \leq m$, hence all polynomials in the Chern classes of $\Lambda_{m}(E)$.

0.3. Tautological module: Elements. Given a family $X / B$ of nodal (possibly pointed) curves, the associated Tautological Module $T_{R}^{m}(X / B)$ (Definition 2.42) is constructed recursively in $m$, grosso modo, as follows (see the body of the paper for details).

- For $m=1$, it equals $R$, a $\mathbb{Q}$-subalgebra of $H^{\bullet}(X)$ containing the relative canonical class $\omega$ as well as any distinguished sections. Here $H^{\bullet}$ denotes any cohomology 
ring coarser than (i.e. admitting a map from) the Chow ring over $\mathbb{Q}$.

- The recursive step. First, decompose the tautological module according to partitions or 'distributions':

$$
T_{R}^{m}(X / B)=\bigoplus_{\mu} T_{R}^{\mu}(X / B)
$$

the sum being over all partitions $\mu$ of weight $m$; thus, it suffices to describe each $\mu$ summand. Then, we parametrize the boundary by a union of families $T(\theta)$ associated to the relative nodes $\theta$ of $X / B$, and for each of those let $X^{\theta} / T(\theta)$ be the corresponding family blown up in $\theta$, which is endowed with a pair of distinguished sections denoted $\theta_{x}, \theta_{y}$, set $R^{\theta}=R\left[\theta_{x}, \theta_{y}\right]$, and define firstly the boundary tautological module of type $\mu$ as

$$
\partial T_{R}^{\mu}=\bigoplus_{\theta} T_{R^{\theta}}^{\mu}\left(X^{\theta} / T(\theta)\right)
$$

(using recursion, we may assume this defined for $\mu$ of weight $<m$ ). Then define for $\mu$ of weight $m$,

$$
T_{R}^{\mu}(X / B)=\left(\operatorname{TS}_{\mu}(R)\right) \oplus\left(\bigoplus_{\substack{\nu \amalg\{n\}=\mu \\ 0<j<n}}\left(\mathbb{Q} F_{j}^{n} \oplus \mathbb{Q} \Gamma^{(m)} F_{j}^{n}\right) \otimes \partial T_{R}^{\nu}(X / B)\right)
$$

in which

- $\operatorname{TS}_{\mu}(R)$, the interior part of the module, is of a purely topological character and can be identified with a formal algebraic construct, an appropriate summand of the 'tensor-symmetric' algebra $T(\operatorname{Sym}(R))$,

- $F_{j}^{n}$ is a formal symbol (for now), called a 'node scroll',

- $\Gamma^{(m)}$ is the discriminant or big diagonal on $X_{B}^{[m]}$, for the purpose of the formula just a formal symbol as well,

- $-\Gamma^{(m)} F_{j}^{n}$ is called a node section.

- We call the two main summands of (0.3.1) the diagonal and node scroll sectors of the tautological module $T_{R}^{\mu}$ and denote them $D T_{R}^{\mu}, N T_{R}^{\mu}$ and similarly $D T_{R}^{m}, N T_{R}^{m}$. NT itself splits as $N F T \oplus N \Gamma T$, node scrolls plus node sections.

The above definition is doubly recursive in the sense that modulo the relatively elementary part $D T_{R}^{m}$, the remaining part $N T_{R}^{m}$ involves tautological modules of lower weight for (boundary) families of lower genus (albeit with more markings). The recursive definition may be replaced by a non-recursive one by working with node polyscrolls, associated to a boundary stratum defined by a collection of nodes rather than a single one.

The tautological module maps to the homology (Chow or ordinary) of the Hilbert scheme, where the diagonal sector maps to cycles living on various diagonal loci (lifted from analogous loci on the symmetric product), and the node scroll sector maps to cycles on certain $\mathbb{P}^{1}$-bundles which live over the boundary and are exceptional for the cycle map. In particular, a zero-dimensional or 'top degree' element $\alpha \in T_{R}^{m}(X / B)$ has a well-defined cycle degree or 'integral' $\int \alpha \in \mathbb{Q}$.

0.4. Tautological module: Discriminant action. Now our first main result, the Tautological module theorem 2.1 , describes the action of $\Gamma^{(m)}$, i.e. the $\mathbb{Q}\left[\Gamma^{(m)}\right]$ module structure, on the $\mathbb{Q}$-vector space $T_{R}^{m}=N T_{R}^{m} \oplus D T_{R}^{m}$. This structure is an 
extension

$$
0 \rightarrow N T_{R}^{m} \rightarrow T_{R}^{m} \rightarrow D T_{R}^{m} \rightarrow 0
$$

where the module structure on the quotient $D T_{R}^{m}$, unrelated to the singularities, is via standard action of the big diagonal in the cohomology of a symmetric product (which can be modelled by a second-order differential operator); the structure on the submodule $N T_{R}^{m}$ is by the standard action (via Grothendieck's formula) of a section $\Gamma^{(m)}$ on the cohomology of a suitable $\mathbb{P}^{1}$-bundle (and it therefore anti-triangular with respect to the $N F T \oplus N \Gamma T$ decomposition). It can be described in terms of discriminant actions of lower weight and lower genus. Also, the 'mixing' part of the action takes $D T_{R}^{m}$ only into the $N F T$ summand of $N T_{R}^{m}$.

0.5. Tautological module: Transfer. As indicated above, the story is completed by the Transfer Theorem 3.4, which computes the transfer (pull-push) operation on $T_{R}^{m-1}(X / B)$ via $X_{B}^{[m, m-1]}$, viewed as a correspondence between $X_{B}^{[m]}$ and $X_{B}^{[m-1]}$, showing in particular that it lands in $T_{R}^{m}(X / B)$.

The conjunction of the Splitting Principle, Module Theorem and Transfer Theorem computes all polynomials in the Chern classes, in particular the Chern numbers, of $\Lambda_{m}(E)$ as $\mathbb{Q}$-linear combinations of tautological classes on $X_{B}^{[m]}$.

0.6. Computation. The calculus of of the discriminant action and Chern polynomials has been implemented (for arbitrary base dimension) on the computer by Gwoho Liu, in the form of a Java program named Macnodal (in honor of MacDonald [13]). See $\S 4.3$ and [12] for details. The results are consistent with Cotteril's [3] results for pencils.

0.7. Punctual transfer. Finally, we discuss an analogue of the tautological module and the transfer for punctual schemes, i.e. those supported at a single point, which are parametrized by the small diagonal $\Gamma_{(m)}$, which itself is a (singular) blowup of $X$. This case is somewhat simpler in its formal aspects but still goes to the heart of the complexities of the Hilbert scheme. It has applications to enumeration of various ramification loci.

0.8. Applications . A number of applications, examples and computations are scattered throughout the paper, especially in $\S \S 2.3,2.4,3.3$ and 4 . In particular, multisecants in nodal families, as mentioned at the beginning, are fully enumerated. In a less elementary vein, the machinery of this paper is projected to be the first step of a project to compute the fundamental class in $\overline{\mathcal{M}}_{g}$ of the locus of curves admitting a $g_{d}^{r}$ for given $r$ and $d$, e.g. a $g_{2}^{1}$ (the hyperelliptics). A baby case (genus 3 ) can be worked out here, thanks to the exceptional luxury that the excess degeneracy is not excessive in dimension. To go further, the idea is to construct an appropriate boundary modification of the Hodge bundle, together with its natural evaluation map to the tautological bundle associated to the canonical bundle, such that the degeneracy locus (in the Hilbert scheme) of this map would consist of the desired $g_{d}^{r}$ locus plus a 'good' excess locus, whose contribution could be computed by Fulton-MacPherson theory. The required modification is nontrivial, especially on the $\Delta_{0}$ boundary component, and is at present known in detail only for $d=2$ (see [16]). Higher-degree cases are work in progress.

I am grateful to Gwoho Liu for many helpful discussions and for creating Macnodal. I also thank Ethan Cotteril for helpful communications about his work, es- 
pecially [3], which provides an alternative method for deriving some of the same enumerative applications in the case of pencils.

1. Preliminaries. This paper is a continuation of our earlier paper [19], whose results, terminology and notations will be used throughout. Some additional terminology and remarks will be given in this section.

1.1. Graph enumeration, generating functions. See the textbooks [1], [7] or [25] for standard techniques and results. We will present some variants of known formulas, which will prove useful in deriving some explicit closed formulas in our Intersection Theory (see especially $§ 2.4$ ).

1.1.1. Simple graphs. We consider connected labelled graphs without loops on a fixed vertex-set $[n]=\{1, \ldots, n\}$. Let $\nu_{n, m}$ be the number of these graphs with $m$ edges, none multiple. We also consider connected 'edge-weighted' graphs on $[n]$, where each edge $e$ is assigned a positive multiplicity $m(e)$. Let $w_{n, m}$ be the weighted number of connected graphs on $[n]$ where the edge multiplicities add up to $m$, i.e. $\sum_{\text {edges }} m(e)=m$, and where the weight of the graph is defined as $\frac{1}{\prod^{m(e) !}}$. Consider the generating functions

$$
T(z, y)=\sum_{n=1}^{\infty} \sum_{m=0}^{\infty} \frac{\nu_{n, m}}{n !} z^{n} y^{m}, T_{b}(u)=\sum_{n=1}^{\infty} \frac{\nu_{n, n-1+b}}{n !} u^{n} .
$$

Here $b$ represents the 1 st Betti number of the graph. Because $\nu_{n, m}=0$ for $m<n-1$, we can write

$$
T(z, y)=\sum_{b=0}^{\infty} y^{b-1} T_{b}(y z)
$$

The classical (and elementary) Riddell-Uhlenbeck formula states that

$$
\exp (T(z, y))=1+\sum_{n=1}^{\infty} \frac{1}{n !} z^{n}(1+y)^{\left(\begin{array}{l}
n \\
2
\end{array}\right)}=1+\sum_{n=1}^{\infty} \frac{1}{n !} \sum_{m=0}^{\left(\begin{array}{c}
n \\
2
\end{array}\right)}\left(\begin{array}{c}
n \\
2 \\
m
\end{array}\right) z^{n} y^{m} .
$$

This follows from the fact that the number of $m$-edge, not necessarily connected graphs on $[n]$ is $\left(\begin{array}{c}n \\ 2 \\ m\end{array}\right)$. In particular for the tree case $(b=0)$ we have (Cayley's result)

$$
T_{0}(z)=\sum_{n=1}^{\infty} \frac{n^{n-2}}{n !} z^{n} .
$$

1.1.2. Edge weighting. We define similarly

$$
W(z, y)=\sum_{b=0}^{\infty} y^{b-1} W_{b}(y z)=\sum_{n=1}^{\infty} \sum_{m=0}^{\infty} \frac{w_{n, m}}{n !} z^{n} y^{m}, W_{b}(u)=\sum_{n=1}^{\infty} \frac{w_{n, n-1+b}}{n !} u^{n} .
$$

Now the total weight of all, not necessarily connected, edge-weighted graphs of total multiplicity $m$ on $[n]$ is

$$
\sum_{m_{1}+\ldots m m\left(\begin{array}{c}
n \\
2
\end{array}\right)=m} \frac{1}{m_{1} ! \ldots m_{\left(\begin{array}{c}
n \\
2
\end{array}\right) !} !}=\frac{1}{m !}\left(\begin{array}{c}
n \\
2
\end{array}\right)^{m} .
$$


It follows similarly that

$$
\exp (W(z, y))=1+\sum_{n=1}^{\infty} \sum_{m=0}^{\infty} \frac{\left(\begin{array}{l}
n \\
2
\end{array}\right)^{m}}{n ! m !} z^{n} y^{m} .
$$

1.1.3. Vertex weighting. This formula admits a useful generalization to vertex-weighted graphs. Suppose vertex $i$ is assigned a fixed weight $p_{i}$ for $i=1, \ldots$, where we may set $p_{i}=0$ for $i>n$ ). The $p_{i}$ are regarded as indeterminates or elements of some $\mathbb{Q}$-algebra $P$. The entire graph is then weighted $\prod_{i<j} \frac{\left(p_{i} p_{j}\right)^{m_{i, j}}}{m_{i, j} !} \in P$, where $m_{i, j}$ is the multiplicity of the $(i, j)$ - edge. Let $w_{S, p ., m}$ be the total weight of all such graphs which are connected and have vertex-set $S$ and total edge multiplicity $m=\sum_{i<j} m_{i, j}$. For a vertex-set $S$ with weights $p$., set

$$
W_{S, p .}(z, y)=\sum_{m=1}^{\infty} \frac{w_{S, p ., m}}{|S| !} z^{S} y^{m}
$$

where $z^{S}=\prod_{i \in S} z_{i}$. The $z_{i}$ are formal variables whose squares are set to zero: $z_{i}^{2}=0$; thus $z^{S} z^{S^{\prime}}=z^{S \cup S^{\prime}}$ whenever $S \cap S^{\prime}=\emptyset$ and otherwise $z^{S} z^{S^{\prime}}=0$. This generating function can be evaluated as follows. Set $f(S, p)=.\sum_{\substack{i<j \\ i, j \in S}} p_{i} p_{j}$ and note that the total weight of all such graphs with vertex-set $S$, without the connectedness hypothesis is

$$
\sum_{\sum m_{i, j}=m} \prod_{\substack{i<j \\ i, j \in S}} \frac{\left(p_{i} p_{j}\right)^{m_{i, j}}}{\prod m_{i, j} !}=\frac{1}{m !} f(S, p .)^{m} .
$$

Then we conclude similarly

$$
\exp \left(\sum_{S} W_{S, p .}\right)=1+\sum_{S, m} \frac{f(S, p .)^{m}}{m !|S| !} z^{S} y^{m} \bmod \left(z_{i}^{2}\right) .
$$

Therefore,

$$
\sum_{S} W_{S, p .}=\sum_{n=1}^{\infty} \frac{(-1)^{n+1}}{n}\left(\sum_{S, m} \frac{f(S, p .)^{m}}{m !|S| !} z^{S} y^{m}\right)^{n} \bmod \left(z_{i}^{2}\right) .
$$

Note that in this formula, each set $S$ on the RHS will be a disjoint union of sets $S$ on the LHS.

1.1.4. Directed case. We now consider a directed analogue of the above. Consider forward-directed edge-weighted graphs on $[m]$. Such a graph is specified by nonnegative integers $e_{j, i}, \forall j<i$ (= number of edges from $j$ to $i$ ), and is assigned a total weight $\frac{1}{\prod e_{j, i} !}$. We will fix the number of edges into $i$ at $k_{i}, i=2, \ldots, m$, i.e. $k_{i}=\sum_{j<i} e_{j, i}$. Let $\vec{w}_{m, k}$. denote the weighted number of these graphs that are connected. On the other hand, the weighted number of all these graphs, possibly disconnected, is, as above

$$
\prod_{i=2}^{m}\left(\sum_{\sum e_{j, i}=k_{i}} \frac{1}{\prod e_{j, i} !}\right)=\prod_{i=2}^{m}\left(\sum_{\sum e_{j, i}=k_{i}} \frac{\left(\begin{array}{c}
k_{i} \\
e_{1, i}, \ldots, e_{i-1, i}
\end{array}\right)}{k_{i} !}\right)=\prod_{i=2}^{m} \frac{(i-1)^{k_{i}}}{k_{i} !} .
$$


Therefore is we define a generating function in $z, y_{2}, \ldots$

$$
\vec{W}\left(z, y_{2}, \ldots\right)=\sum_{m=2}^{\infty} \sum_{k_{1}, \ldots k_{m}} \frac{\vec{w}_{m, k}}{m !} z^{m} y_{2}^{k_{2}} \ldots y_{m}^{k_{m}}
$$

then it follows that

$$
\exp \left(\vec{W}\left(z, y_{2}, \ldots\right)\right)=1+\sum_{m, k} \frac{1}{m !} \prod_{i=2}^{m} \frac{(i-1)^{k_{i}}}{k_{i} !} z^{m} y_{2}^{k_{2}} \ldots y_{m}^{k_{m}} .
$$

Thus we may consider the $\vec{w}_{m, l}$. as known.

1.2. Products, diagonals, partitions. The intersection calculus we aim to develop is couched in terms certain diagonal-like loci on products, defined in the general case in terms of partitions. To facilitate working with these loci systematically, we now establish some conventions, notations and simple remarks related to partitions. Our viewpoint on partitions is influenced by the fact that we will mainly use them to define 'diagonal' conditions, so in particular singleton blocks are essentially insignificant.

1.2.1. b-partitions. By a block partition or b-partition (aka labelled partition) (I.) of weight $m$ and length $r$ we mean an expression

$$
\{1, \ldots, m\}=I_{1} \coprod \ldots \coprod I_{r}, \forall I_{j} \neq \emptyset
$$

If a b-partition $(I$.$) is such that all its blocks except for one of them, say I$, are singletons, we will denote $(I$.$) as (I)$ or $(I, m]$. Given a set $X$ (or an object in a category with products - the modifications for this case are left to the reader), a bpartition $(I$.$) of weight m$ defines an ordered 'polydiagonal' subset of the (Cartesian) product $X^{m}$, which will be denoted by $X^{(I .)}$ or $O D_{(I .)}$ or, if the dependence on $X$ must be explicated, $O D_{(I .), X}$ : in the case where $X$ is a set, we identify $X^{m}$ with the set of functions $\{1, \ldots, m\} \rightarrow X$, and then $X^{(I .)}=O D_{(I .)} \subset X^{m}$ consists of the functions constant on each block. It is the image of an injection $X^{r} \rightarrow X^{m}$ and will sometimes also be identified with that injection.

1.2.2. Partitions. A b-partition $(I$. ) determines an ordinary partition of the same weight, viz. $(|I|$.$) , which we prefer to view via the corresponding 'length distri-$ bution'. Thus the length distribution associated to a b-partition (I.) is the function $\mu: \mathbb{N} \rightarrow \mathbb{Z}_{\geq 0}$ defined by

$$
n \mapsto\left|\left\{j:\left|I_{j}\right|=n\right\}\right|
$$

We call a function $\mu: \mathbb{N} \rightarrow \mathbb{Z}_{\geq 0}$ either a distribution or partition. This is the same thing as 'partition' in the usual sense: in the usual partition notation, the partition corresponding to $\mu$ is $\left(\ldots, n^{\mu(n)}, \ldots, 2^{\mu(2)}, 1^{\mu(1)}\right)$. A distribution has weight $|\mu|=w(\mu)=$ $\sum n \mu(n)=m$ degree $d(\mu)=\sum_{n>0}(n-1) \mu(n)$ and length $\ell(\mu)=|\{n: \mu(n)>0\}|$.

The length distribution of a b-partition of weight $m$ has weight $m$ and conversely, any distibution of weight $m$ is the length distribution of some b-partition of weight $m$. Two b-partitions are said to be equivalent if their distributions are the same or equivalently, if they differ by a permutation of $1, \ldots, m$. A distribution is viewed essentially as a collection of block sizes, and will often be specified by specifying 
the non-singleton block sizes: e.g. $(n)$ for $n>1$ refers to a distribution (of some weight $m \geq n$ ) with unique nonsingleton block of size $n$. A distribution $\mu$ defines a polydiagonal or polyblock diagonal

$$
D_{\mu}=D_{\mu, X}=\prod_{n} X^{(\mu(n))} \hookrightarrow X^{(w(\mu))}
$$

where $X^{(k)}$ is the $k$ th symmetric product. The embedding is defined by repeating an element in the $n$th factor, i.e. $X^{(\mu(n))}, n$ times, i.e.

$$
\left(\sum_{i=1}^{\mu(n)} x_{i, n}: n=1,2, \ldots\right) \mapsto \sum_{n} \sum_{i=1}^{\mu(n)} n x_{i, n} .
$$

When $X$ has a well-defined dimension $\operatorname{dim}(X)$, the codimension of $D_{\mu}$ in $X^{(w(\mu))}$ is $d(\mu) \operatorname{dim}(X)$. As above, $D_{\mu}$ may be viewed either as a locus or a map. We will write $D_{(n), m}$ for $D_{\mu}$ where $\mu$ is the unique distribution of weight $m$ with unique nonsingleton block of size $n$. Also, we will denote by $1^{m}$ the unique distribution of weight $m$ supported on $\{1\}$, whose associated polyblock diagonal if $X^{(m)}$ itself.

The following is an easy remark.

LEMMA 1.1. For a b-partition (I.) with corresponding distribution $\mu$, the degree of the map $O D_{(I .)} \rightarrow D_{\mu}$ is

$$
a(\mu):=\prod_{n} \mu(n) !
$$

1.2.3. Union operation. Now, we will need some operations on b-partitions and associated distributions. Let $u_{r, s}(I$.) be the b-partition obtained from $(I$.$) by$ deleting the $r$ th and $s$ th blocks, $r \neq s$ and inserting their union. We let $u_{a, b}(\mu)$ be the corresponding operation on distributions, which corresponds to deleting blocks of size $a, b$ and inserting a block of size $a+b$; by definition, $u_{a, b}(I)=.\emptyset$ unless $I$. contains blocks of sizes $a, b$ (two blocks of size $a$, if $a=b$ ); in other words,

$$
u_{a, b}(\mu)=\left\{\begin{array}{l}
\mu-1_{a}-1_{b}+1_{a+b}, \mu \geq 1_{a}+1_{b} \\
\emptyset, \text { otherwise }
\end{array}\right.
$$

Here $1_{a}$ is the indicator (characteristic) function of $a$. i.e.

In the geometric setting, $u_{a, b}$ correspond to intersecting with a suitable diagonal,

$$
D_{u_{r, s}(I .)}=D_{I .} \cap D_{i, j}
$$

where $i \in I_{r}, j \in I_{s}$ are arbitrary and $D_{i, j}$ is the pullback of the diagonal from the $i, j$ factors.

1.3. Diagonal operators on tensors. Given a topological space $X$, the polydiagonals of its symmetric products are reflected algebraically in the (co)homology of these symmetric products. The algebraic structures that result can be defined purely algebraically, which is the purpose of this section. 
1.3.1. Tensymmetric algebra. Let $R$ be a commutative unitary graded $\mathbb{Q}$ algebra. An example to keep in mid thoughout is a subalgebra of the cohomology of a topological space (e.g. manifold). Consider the so-called 'tensymmetric' algebra

$$
\operatorname{TS}(R)=\bigotimes_{n=\infty}^{1} \operatorname{Sym}^{\bullet}(R)
$$

Here and elsewhere, unless otherwise specified Sym means $\operatorname{Sym}_{\mathbb{Q}}$. Let $\alpha$. be a simple (decomposable) element in this algebra. Then $\alpha$ can be written as

$$
\alpha .=\bigotimes_{n} \prod_{i=1}^{\mu(n)} \alpha_{n, i} .
$$

Here the second product is the formal one in $\operatorname{Sym}^{\bullet}(R)$ (rather than the one in $R$, which will be denoted $\cdot_{R}$ or $\prod_{R}$ ); when in doubt, the product in Sym will be denoted .S. In the geometric situation, the products in Sym and $\otimes$ both correspond to external cup products, and then will often be denoted by $\star$. The function $\mu$ is a length distribution, i.e. a finitely-supported function from the positive integers to the nonnegative integers and we call $\mu$ the distribution associated to $(\alpha$.). Thus $\alpha$. is a tensor product of symmetric tensors, with the one in position $n$ having (tensor) degree $\mu(n)$. This yields a 'grading by distribution':

$$
\begin{array}{r}
\mathrm{TS}(R)=\bigoplus_{\mu} \operatorname{TS}_{\mu}(R), \\
\operatorname{TS}_{\mu}(R)=\bigotimes_{n} \operatorname{Sym}^{\mu(n)}(R) .
\end{array}
$$

We define the weight of an element $\alpha \cdot \operatorname{TS}_{\mu}(R)$ as that of the associated distribution, i.e. $w(\alpha)=.\sum n \mu(n)$. Of course, in any simple $\alpha$., all but finitely many tensor factors (or ' $n$-block factors', we we shall call them) equal 1 . Via the natural inclusion $\operatorname{Sym}^{\mu(n)}(R) \rightarrow R^{\otimes \mu(n)}, \alpha$ may be viewed as an element of

$$
\mathrm{TI}_{\left(I_{1}, \ldots, I_{k}\right)}(R):=R^{\otimes k}
$$

for any b-partition $(I$.$) so that (|I|)=.\mu$. Thus, we may define the 'inflated tensor algebra' as

$$
\mathrm{TI}(R)=\bigoplus_{(I .)} \mathrm{TI}_{(I .)}(R), \operatorname{TI}_{\left(I_{1}, \ldots, I_{k}\right)}(R)=R^{\otimes k}
$$

Then we have a natural inclusion

$$
\mathrm{TS}(R) \rightarrow \operatorname{TI}(R)
$$

which takes $\operatorname{TS}_{\mu}(R)$ to $\underset{|I .|=\mu}{\bigoplus} \mathrm{TI}_{(I .)}(R)$. In the other direction, there is also a natural symmetrization map

$$
\mathrm{TI}(R) \rightarrow \mathrm{TS}(R)
$$

which takes $\mathrm{TI}_{(I .)}(R)$ to $\mathrm{TS}_{|I .|}(R)$. 
1.3.2. Norm operator. For an element $\theta \in R$, we denote by

$$
[m]_{*}(\theta) \in \operatorname{Sym}^{m}(R)=\operatorname{TS}_{1^{m}}(R)
$$

the element (symmetric tensor)

$$
\theta .1^{m-1}
$$

and more generally by

$$
[m]_{*}^{s}(\theta) \operatorname{TS}_{1^{m}}(R)
$$

the element

$$
\theta^{s} \cdot 1^{m-s} \text {. }
$$

This called the $m$-th topological norm of $\theta$. See $\S 1.6$ for a geometric interpretation.

1.3.3. Diagonal operators. We now define a weight-preserving 'projection'

$$
D_{\bullet}^{\dagger}: \operatorname{Sym}^{\bullet}(R) \rightarrow \operatorname{TS}(R) .
$$

This is a vector with components $D_{\mu}^{\dagger}, \mu$ ranging over all distributions. Each $D_{\mu}^{\dagger}$ is defined as follows. Let $m=w(\mu)=\sum n \mu(n)$ for a distribution $\mu$ and consider a decomposable element $\beta \in \operatorname{Sym}^{m}(R)$. Then $\beta$ comes from many elements of the form

$$
\beta \ldots=\bigotimes \bigotimes_{n} \prod_{i=1}^{\mu(n)} \prod_{k=1}^{n} \beta_{n, i, k}
$$

(the two internal products are in $\operatorname{Sym}^{\bullet}$ ). We call such $\beta \ldots$ a lift of $\beta$. Then let $D_{\mu}^{\dagger}(\beta)$ as the following sum over all possible lifts $\beta \ldots$ of the given $\beta$ :

$$
D_{\mu}^{\dagger}(\beta)=\sum_{\beta \ldots \mapsto \beta}\left(\bigotimes_{n} \prod_{i=1}^{\mu(n)}\left(\prod_{k=1}^{n} R \beta_{n, i, k}\right)\right)
$$

where $\prod_{R}$ means product in $R$ and the middle product is again product in $\mathrm{Sym}^{\bullet}$. Each $D_{\mu}^{\dagger}$ is a projection in the sense that it admits a right inverse. This right inverse is the natural 'forgetful' map

$$
\begin{array}{r}
D_{\mu}: \operatorname{TS}_{\mu}(R) \rightarrow \operatorname{Sym}^{m}(R), m=w(\mu), \\
\bigotimes_{n} \prod_{i=1}^{\mu(n)} \alpha_{n, i} \mapsto \prod_{n} \prod_{i=1}^{\mu(n)} 1^{n-1} \alpha_{n, i}
\end{array}
$$

(internal product is the product in Sym). Assembling these together, we get a map

$$
D_{\bullet}[]=\bigoplus_{\mu} D_{\mu}[]: \operatorname{TS}(R) \rightarrow \operatorname{Sym}^{\bullet}(R) .
$$

Often $R$ will be a graded ring, which naturally induces a gradation on $\operatorname{TS}(R)$, said to be by degree (not to be confused with weight). If $R$ has top piece $R^{d}$ endowed with a linear map $\int: R^{d} \rightarrow \mathbb{Q}$, extended by zero to $R$, then $\int$ extends to $\operatorname{TS}(R)$ by multiplicativity, i.e. for $\alpha$ decomposable,

$$
\int(\alpha .)=\prod_{n, i}\left(\int \alpha_{n, i}\right)
$$

which of course depends only on the degree- $d$ component of each $\alpha_{n, i}$ and vanishes if one of these components does. 
1.3.4. Duality. If $R$ is $\mathbb{Q}$-self-dual, $D_{\mu}^{\dagger}$ also admits a more useful (weight- preserving) 'Gysin adjoint'

$$
D_{\mu \dagger}: \operatorname{TS}_{\mu}(R) \rightarrow \operatorname{Sym}^{\bullet}(R)
$$

defined as follows. Let $\operatorname{Sym}^{n}(R) \rightarrow R$ be the multiplication map, which by duality corresponds to a map

$$
J_{n}: R \rightarrow \operatorname{Sym}^{n}(R)
$$

Then define

$$
D_{\mu \dagger}\left(\bigotimes_{n} \prod_{i=1}^{\mu(n)} \alpha_{n, i}\right)=\prod_{n} \prod_{i=1}^{\mu(n)} J_{n}\left(\alpha_{n, i}\right)
$$

Elements in the image of $D_{\mu \dagger}$ are called polyblock diagonal classes of type $\mu$.

1.3.5. Ordered analogue. All of the above admit an ordered analogue, where both $\operatorname{Sym}(R)$ and $\operatorname{TS}(R)$ are replaced by the tensor algebra $\otimes R$, and partitions $\mu$ are replaced by b-partitions $\left(I\right.$.) The analogue of the map $D_{\mu}$ is the map

$$
\begin{array}{r}
D_{\left(I_{1}, \ldots, I_{k}\right)}: \bigotimes^{m} R \rightarrow \bigotimes^{k} R, m=|I .|, \\
\alpha_{1} \cdots \alpha_{m} \mapsto \prod_{j=1}^{k}\left(\prod_{i \in I_{j}} R \alpha_{i}\right)
\end{array}
$$

that replaces each tensor product inside a block by the corresponding $R$-product.

1.3.6. Interpretation. Given a space $X$, a partition $\mu$ of weight $m$ corresponds to a polyblock diagonal subspace $d_{\mu}$, a cartesian product of symmetric products of the symmetric product $X^{(m)}$. These assemble together to a finite-to-one map

$$
d_{\bullet m}=\coprod_{w(\mu)=m} d_{\mu} \rightarrow X^{(m)} .
$$

If $R$ represents some kind of cohomology ring, e.g. the Chow ring on a variety, then the Gysin map associated to $d_{\mu}$ is $D_{\mu \dagger}$. In particular, $* 1$ is the class of a point, $J_{n}$ is the Gysin map for the embedding of the small diagonal in a symmetric product. Each $\alpha_{n, i}$ is considered as living on a small diagonal $X \subset \operatorname{Sym}^{n}(X)$ and $\prod_{i=1}^{\mu(n)} \alpha_{n, i}$ lives on $X^{(\mu(n))} \subset X^{(n \mu(n))}$. The map $D_{\mu}^{\dagger}$ is the pullback map induced by the inclusions $d_{\mu} \rightarrow \operatorname{Sym}^{\bullet}(X)$, while $D_{\mu}$ is a natural right inverse for it.

1.4. Discriminant operator. Our aim now to define a 'discriminant' operation on $\operatorname{TS}(R)$ that corresponds to intersecting with the big diagonal for $X$ smooth. As part of our intersection calculus for Hilbert schemes, we will later derive a formula for intersecting with the discriminant polarization of which this operation will form the 'classical' part.

To this end we first define an operation $u_{n_{1}, n_{2}}$ on $\operatorname{TS}(R)$ (not preserving individual $\left.\mathrm{TS}_{\mu}(R)\right)$ that corresponds to uniting two blocks of sizes $n_{1}, n_{2}$, similar to the 
corresponding definition for polyblocks. The definition is:

$$
\begin{aligned}
& \forall \alpha=\bigotimes_{n} \prod_{i=1}^{\mu(n)} \alpha_{n, i} \in \operatorname{TS}_{\mu}(R), \forall n_{1} \neq n_{2}: \\
& u_{n_{1}, n_{2}: \mu}(\alpha .)= \\
& \sum_{i=1}^{\mu\left(n_{1}\right)} \sum_{j=1}^{\mu\left(n_{2}\right)} \ldots \otimes \alpha_{n_{1}+n_{1}, 1} \ldots \alpha_{n_{1}+n_{2}, \mu\left(n_{1}+n_{2}\right)}\left(\alpha_{n_{1}, i \cdot R} \alpha_{n_{2}, j}\right) \otimes \ldots \hat{\alpha}_{n_{1}, i} \ldots \hat{\alpha}_{n_{2}, j} \ldots \\
& u_{n, n: \mu}(\alpha .)=\sum_{1 \leq i<j \leq \mu(n)} \ldots \otimes \alpha_{2 n, 1} \ldots \alpha_{2 n, \mu(2 n)}\left(\alpha_{n, i \cdot R} \alpha_{n, j}\right) \otimes \ldots \hat{\alpha}_{n, i} \ldots \hat{\alpha}_{n, j} \ldots
\end{aligned}
$$

(here . means 'omit', as usual). In other words, for the case $n_{1} \neq n_{2}$ : omit in all possible ways one alpha factor from each of the $n_{1}$ and $n_{2}$ block subproducts and insert their $R$-product in the $n_{1}+n_{2}$-block subproduct. This defines a map

$$
u_{n_{1}, n_{2}: \mu}: \operatorname{TS}_{\mu}(R) \rightarrow \mathrm{TS}_{u_{n_{1}, n_{2}}(\mu)}(R)
$$

where $u_{n_{1} . n_{2}}(\mu)$ is as in (1.2.8).

In particular, for the 'trivial' partition $\mu=\left(1^{m}\right)$, we get

$$
u_{1,1,\left(1^{m}\right)}\left(\alpha_{1} \ldots \alpha_{m}\right)=\sum_{i<j}\left(\alpha_{i \cdot R} \alpha_{j}\right) \otimes\left(\alpha_{1} \ldots \hat{\alpha_{i}} \ldots \hat{\alpha_{j} \ldots \alpha_{m}}\right) \in R \otimes \operatorname{Sym}^{m-2}(R) .
$$

ExAmPle. For $\mu=\left(2,1^{2}\right)=(2 \mapsto 1,1 \mapsto 2)$, we have

$$
u_{2,1, \mu}\left(\alpha_{2} \alpha_{1,1} \alpha_{1,2}\right)=\left(\alpha_{2 \cdot R} \alpha_{1,1}\right) \alpha_{1,2}+\left(\alpha_{2 \cdot R} \alpha_{1,2}\right) \alpha_{1,1}
$$

The unspecified product is that of Sym, of course. In particular,

$$
\begin{gathered}
u_{2,1, \mu}\left(\alpha_{2} 1_{R}^{2}\right)=2 \alpha_{2} 1_{R} \\
u_{1,1, \mu}\left(\alpha_{2} \alpha_{1,1} \alpha_{1,2}\right)=\alpha_{2}\left(\alpha_{1,1 \cdot R} \alpha_{1,2}\right) .
\end{gathered}
$$

In a rather more general vein, we can associate a similar operation to partition $\nu \leq \mu$ :

$$
\begin{array}{r}
u_{\nu: \mu}: \operatorname{TS}_{\mu} \rightarrow \operatorname{TS}_{\mu-\nu^{\prime}}, \nu^{\prime}(n):=\min (\nu(n)-1,0), \\
\bigotimes_{n} \prod_{n, j} \mapsto \bigotimes_{n}\left(\sum_{\left|A_{n}\right|=\nu(n)}\left(\prod_{j \in A_{n}} R \alpha_{n, j}\right)\left(\prod_{j \notin A_{n}} \alpha_{n, j}\right)\right)
\end{array}
$$

This corresponds to uniting a set of blocks corresponding to $\nu_{i}$, for $i=1, \ldots, r$, to a single block of size $\left|\nu_{i}\right|$, and $R$-multiplying the corresponding $\alpha$ factors.

Similarly, if $\nu_{1}, \ldots, \nu_{r}$ are partitions with $\sum \nu_{i} \leq \mu$, we can define inductively

$$
\begin{array}{r}
u_{\nu_{1} ; \ldots ; \nu_{r}: \mu}: \operatorname{TS}_{\mu}(R) \rightarrow \operatorname{TS}_{\mu(r)}(R), \\
u_{\nu_{1} ; \ldots ; \nu_{r}: \mu}=u_{\nu_{1} ; \ldots ; \nu_{r-1}: \mu^{\prime}} \circ u_{\nu_{r}: \mu}, \mu^{\prime}=\mu-\nu_{r}^{\prime} .
\end{array}
$$

There is a (simpler) ordered analogue of this, which takes the form

$$
\begin{array}{r}
o u_{i, j}: \bigotimes^{k} R \rightarrow \bigotimes^{k-1} R, i<j \\
\alpha_{1} \ldots \alpha_{i} \ldots \alpha_{j} \ldots \alpha_{k} \mapsto \alpha_{1} \ldots\left(\alpha_{i \cdot R} \alpha_{j}\right) \ldots \hat{\alpha_{j} \ldots \alpha_{k}}
\end{array}
$$


Next, define an operation corresponding to multiplication by a fixed ring element within a block of size $n$ : for an element $\omega \in R$, define

$$
u_{n, \omega, \mu}(\alpha .)=\sum_{i=1}^{\mu(n)} \ldots \otimes \ldots \hat{\alpha}_{n, i} \cdot S\left(\alpha_{n, i \cdot R} \omega\right) \ldots
$$

In other words, replace in all possible ways an element in the $n$-block by its $R$-product with $\omega$ (this might be called $R$-multiplication by $\omega$, extended to $\operatorname{TS}(R)$ as a 'derivation inside the $n$-block'). We can similarly define for any $\mathbb{Q}$-linear map $g: R \rightarrow R$,

$$
u_{n, g, \mu}(\alpha .)=\sum_{i=1}^{\mu(n)} \ldots \otimes \ldots \hat{\alpha}_{n, i} \cdot S g\left(\alpha_{n, i}\right) \ldots,
$$

(i.e. $g$ extended as a derivation or 'interior multiplication by $g$ in the $n$-block). Again, there is a simple ordered analogue, given by

$$
o u_{i, g}\left(\alpha_{1} \ldots \alpha_{i} \ldots \alpha_{k}\right)=\alpha_{1} \ldots g\left(\alpha_{i}\right) \ldots \alpha_{k} .
$$

When there is no confusion, we will denote $o u_{i, g}$ by $o u_{I_{i}, g}$.

Finally, in terms of these, define the 'discriminant' operator on $\operatorname{TS}_{\mu}(R)$ by

$$
\begin{aligned}
\operatorname{Dsc}_{\mu} & : \operatorname{TS}_{\mu}(R) \rightarrow \operatorname{TS}(R), \\
\operatorname{Dsc}_{\mu} & =\sum_{n_{1} \geq n_{2}} n_{1} n_{2} u_{n_{1}, n_{2}, \mu}
\end{aligned}
$$

In particular, set

$$
\operatorname{Dsc}^{(m)}=\operatorname{Dsc}_{\left(1^{m}\right)} .
$$

Also set

$$
U_{\omega, \mu}(\alpha .)=\sum_{n}\left(\begin{array}{l}
n \\
2
\end{array}\right) u_{n, \omega, \mu}(\alpha .), \alpha . \in \operatorname{TS}_{\mu}(R)
$$

These assemble together to maps

$$
\text { Dsc, } U_{\omega}: \operatorname{TS}(R) \rightarrow \operatorname{TS}(R)
$$

and similarly, an ordered version on the inflated tensor product $\mathrm{TI}(R)$ :

$$
O \mathrm{Dsc}=\sum_{i<j} u_{i, j}, O U_{\omega}=\sum_{i} o u_{i, \omega} .
$$

For future reference, it is important to note that we can write

$$
O \text { Dsc }-O U_{\omega}=\sum_{i<j} D_{i, j}
$$

where $D_{i, j}$ acts on $\operatorname{TI}_{(I .)}(R)$ as $u_{i, j}$ if $i, j$ are in different blocks of $(I$.$) , and as o u_{k, \omega}$ if $i, j$ are both in the $k$-th block $I_{k}$.

The motivation for this definition is the following elementary result, which could be deduced from Macdonald's work [13]. Recall first (see $\S 1.5$ below) that if $X$ is 
a smooth curve over $\mathbb{C}$, there is a 'half discriminant' class, which we abusively call discriminant, $\operatorname{Dsc}^{(m)}$ on the symmetric product $X^{(m)}$, which is half the class of the big diagonal $D^{(m)}$ (locus of nonreduced cycles), and whose pullback to the cartesian product coincides with the big diagonal there (with multiplicity 1). Similarly, there is a discriminant class on a cartesian product of symmetric products $\prod X^{\left(m_{i}\right)}$.

Lemma 1.2. Let $X$ be a smooth curve with canonical class $\omega, R=H \cdot(X)$. Then

$i$. The cup-product action of the discriminant on $H \cdot\left(\prod X^{(\mu(n))}\right)=\operatorname{TS}_{\mu}(R)$ is given by $\operatorname{Dsc}_{\mu}-U_{\omega, \mu}$.

ii. The cup product action of the discriminant on polyblock diagonal classes is given by

$$
\left[\operatorname{Dsc}^{(m)}\right] \cup\left[D_{\mu \dagger}(\alpha .)\right]=\left[D_{\bullet}\left(\operatorname{Dsc}_{\mu}(\alpha .)-U_{\omega, \mu}(\alpha .)\right)\right], \alpha . \in \operatorname{TS}_{\mu}(R)
$$

Proof. The second part is just an elaboration of the first, so it suffices to prove (1.4.14). Since we are working over $\mathbb{Q}$, it suffices to prove both sides are equal after pullback to the Cartesian product where the pullback of $\operatorname{Dsc}^{(m)}$ is the big diagonal (multiplicity $1(!)$ ), and splits as a sum of the diagonals pulled back from $X \times X$, namely $\sum_{a<b} D_{a, b}$, and $\alpha$. is replaced by a class $\alpha_{(I .)}$ on an ordered polyblock diagonal. Then clearly those $a, b$ in different blocks of sizes $n_{1}, n_{2}$ (the sizes may be different or not) give rise to $u_{n_{1}, n_{2}}$, while those in the same block of size $n$ give rise to $u_{n, \omega}$.

This result remains true, in fact, when $X$ is nodal (as follows, e.g. from the discussion in $\S 2.2$, or by an elementary dimension-counting argument). However, it is of little interest in that case because of the lack of geometric meaning of the symmetric products. On the other hand, one of the main ingredients of our intersection calculus, to be developed starting in the next section, is an analogue of the Lemma for Hilbert schemes of families of nodal curves (see Proposition 2.20), where the two sides of (1.4.14) are not equal but differ by an 'exceptional' class called a node scroll class. The device of pulling back to an ordered version will be used there too.

1.5. (Half-) discriminant. Let $X / B$ be a family of smooth curves and $D^{(m)}$ the big diagonal (or discriminant) in the relative symmetric product $X_{B}^{(m)}$, i.e. $D_{\mu} \cap X_{B}^{(m)}$ for $\mu=(2 \mapsto 1,1 \mapsto m-2)=\left(2,1^{m-2}\right.$ ) (also written simply as $\left.(2)\right)$. This is a reduced Cartier divisor, defined locally by the discriminant function which is a polynomial in the elementary symmetric functions of a local parameter of $X / B$. The associated line bundle $\mathcal{O}\left(D^{(m)}\right)$ is always canonically divisible by 2 as line bundle. Its half is denoted $h=\operatorname{Dsc}^{(m)}$. One way to see this is to note that $D^{(m)}$, which is the branch locus of $\varpi: X_{B}^{m} \rightarrow X_{B}^{(m)}$, is also the branch locus of a flat (albeit singular) double cover

$$
\epsilon: X_{B}^{\odot m} \rightarrow X_{B}^{(m)}
$$

where $X_{B}^{\odot m}=X_{B}^{m} / \mathfrak{A}_{m}$ is the 'orientation product', quotient of the cartesian product by the alternating group, which generically parametrizes an $m$ - cycle together with an orientation. Then $h$ is defined by

$$
\epsilon_{*} \mathcal{O}_{X_{B}^{\odot m}}=\mathcal{O}_{X_{B}^{(m)}} \oplus h^{-1}
$$

(i.e. $h^{-1}$ is canonically the kernel of the trace map $\epsilon_{*} \mathcal{O}_{X_{B}^{\odot m}} \rightarrow \mathcal{O}_{X_{B}^{(m)}}$ ). Indeed $\epsilon^{*} h$ is precisely the (reduced) ramification divisor of $\epsilon$, which is half of $\epsilon^{*} D^{(m)}$. In particular, note that $\epsilon^{*} h$ is effective. 
An explicit formula for the discriminant- which extends to Hilbert schemes as well- is the following (see [11], p.8): let $Z \subset X^{(m)} \times X$ be the tautological subscheme and $A=p_{1 *} \mathcal{O}_{Z}$. The analogous object on the Hilbert scheme is what we call the tautological sheaf associated to the trivial bundle and denote by $\Lambda_{m}\left(\mathcal{O}_{X}\right)$ (see $\S 3.2$ ). Then $A$ is endowed with a trace pairing, whence a map $A \rightarrow A^{*}$ which drops rank precisely on $D^{(m)}$, hence $\left[D^{(m)}\right]=-2 c_{1}(A)$. Therefore a half-discriminant can be defined by

$$
\operatorname{Dsc}^{(m)}=-c_{1}(A)=-c_{1}\left(\Lambda_{m}\left(\mathcal{O}_{X}\right)\right)
$$

The same formula applies to define the discriminant $\Gamma^{(m)}$ on the Hilbert scheme $X_{B}^{[m]}$ (at least for any family $X / B$ of nodal curves).

Though not important for our purposes it amusing to note that the two definitions of discriminant agree. This follows from the fact that on the orientation product $X_{B}^{\odot m}$, the pullback $\epsilon^{*}\left(\operatorname{det}\left(\Lambda_{m}\left(\mathcal{O}_{X}\right)\right)\right)$ is an ideal sheaf (hence, it is the ideal sheaf of $\left.\epsilon^{*}\left(D^{(m)}\right)_{\text {red }}\right)$ : the map $\epsilon^{*}\left(\operatorname{det}\left(\Lambda_{m}\left(\mathcal{O}_{X}\right)\right)\right) \rightarrow \mathcal{O}_{X \odot m}$ is given by first mapping over $X_{B}^{m}$ :

$$
f_{1} \wedge \ldots \wedge f_{m} \mapsto \sum_{\sigma \in \mathfrak{S}_{m}} \operatorname{sgn}(\sigma) \sigma^{*} f_{1} \cdots \sigma^{*} f_{m}
$$

then noting that this is $\mathfrak{A}_{m}$-invariant on $X_{B}^{m}$, hence descends to $X_{B}^{\odot m}$.

1.6. Norm. For a line bundle $L$ on a family of smooth curves $X / B$, we denote by $[m]_{*}(L)$ its norm on the symmetric product $X^{(m)}$, defined by

$$
[m]_{*}(L)=c_{1}\left(p_{1 *}\left(p_{2}^{*} L \otimes \mathcal{O}_{Z}\right)\right)+\operatorname{Dsc}^{(m)}=c_{1}\left(\Lambda_{m}(L)\right)+\operatorname{Dsc}^{(m)}
$$

(notations as above). For an effective Cartier divisor $D$ on $X$, the norm of $\mathcal{O}(D)$ is

$$
[m]_{*}(D)=p_{1 *}\left(p_{2}^{*}(D) \cdot Z\right)
$$

(direct image as cycle). To see this fact (just the Riemann-Roch for the finite map $\left.Z \rightarrow X_{B}^{[m]}\right)$, use the exact sequence

$$
0 \rightarrow \Lambda_{m}(\mathcal{O}(-D)) \rightarrow \mathcal{O}_{X} \rightarrow \mathcal{O}_{D} \rightarrow 0
$$

Thus, $[m]_{*}(D)$ is a divisor supported on the locus of cycles meeting $D$. Note again that the same formula (1.6.18) defines the norm in the Hilbert scheme setting.

In terms of cohomology, the class $[m]_{*}(D)$ for $D$ effective is just the class corresponding to $[D] 1^{m-1}$ under the identification of $H \cdot\left(\operatorname{Sym}^{m}(X)\right)$ with $\operatorname{Sym}^{m}(H \cdot(X))$.

Similarly, we set, for $s \leq m$,

$$
[m]_{*}^{s}(D)=\varpi_{*}\left(p_{1}^{*}(D) \ldots p_{s}^{*}(D)\right) .
$$

This corresponds to $[D]^{s} 1^{m-s}$.

These constructions are compatible with that of $\$ 1.3 .2$, in the sense that the cohomology class of $[m]_{*}^{s}(D)$ is $[m]_{*}^{s}([D])$. This is clear from the above description.

REMARK 1.3. Another formula for $\operatorname{Dsc}^{(m)}$ (see [21], Corollary 2.1) is

$$
\operatorname{Dsc}^{(m)}=[m]_{*}\left(\omega_{X / B}\right) \otimes \omega_{X_{B}^{(m)} / B}^{-1} .
$$


To prove this up to numerical equivalence it suffices to show that the pullback both sides on the relative Cartesian product $X_{B}^{m}$ are isomorphic. This is proved by applying the Riemann-Hurwitz formula for smooth varieties to $\varpi: X_{B}^{m} \rightarrow X_{B}^{(m)}$.

REMARK 1.4. In Macdonald's development [13] of intersection theory on symmetric products of a single smooth curve $X / \mathbb{C}$, as expounded in [2], Ch. 8 , is based on the classes $[m]_{*}[p t]=: \theta_{m}$ and $\left[\Theta_{m}\right]$ which is the pullback of the theta-divisor $\Theta(m)$ on the Jacobian $J(m)$ via the Abel-Jacobi map (his notation is different). $\Theta(g-1)$ may be realized as the locus of effective line-bundles of degree $g-1$ in $J(g-1)$. In fact, $\left[\Theta_{m}\right]$ and $\Gamma^{(m)}:=\operatorname{Dsc}^{(m)}$ are related by:

$$
\left[\Theta_{m}\right]=(g+m-1) \theta_{m}-\Gamma^{(m)} .
$$

This may be seen as follows. For $m \geq g$, let $L$ be any line bundle of degree $m+g-1$, hence with $m$ sections. Then the map

$$
\begin{gathered}
X^{(m)} \rightarrow J(g-1), \\
z \mapsto L(-z)
\end{gathered}
$$

is surjective and pulls back $\Theta(g-1)$ to $\Theta_{m}$. Therefore, $\left[\Theta_{m}\right]$ is the degeneracy class of the natural evaluation map

$$
H^{0}(L) \otimes \mathcal{O} \rightarrow \Lambda_{m}(L)
$$

This yields (1.6.22).

For $m<g, \Theta_{m}$ is induced by $\Theta_{g}$ via $X^{(m)} \rightarrow X^{(m)}+(g-m)[p t] \subset X^{(g)}$, and $\Gamma^{(g)} \cdot X^{(m)}=\Gamma^{(m)}+(g-m) \theta_{m}$, hence (1.6.22) follows again.

In any event, passing between $\Theta$ and $\Gamma$-based theories is a matter of simple change of variable.

1.7. Boundary data. Let $\pi: X \rightarrow B$ now denote an arbitrary flat family of nodal curves of arithmetic genus $g$ over an irreducible base, with smooth generic fibre. In order to specify the additional information required to define a node scroll, we make the following definition.

Definition 1.5. A boundary datum for $X / B$ consists of

$i$. an irreducible variety $T$ with a map $\delta: T \rightarrow B$ unramified to its image, where the image is a component of the boundary, i.e. the locus in $B$ parametrizing singular curves;

ii. a 'relative node' over $T$, i.e. a lifting $\theta: T \rightarrow X$ of $\delta$ such that each $\theta(t)$ is a node of $X_{\delta(t)}$;

iii. a labelling, continuous in $t$, of the two branches of $X_{\delta(t)}$ along $\theta(t)$ as $x$-axis and $y$-axis.

Given such a datum, the associated boundary family $X_{T}^{\theta}$ is the normalization (= blowup) of the base-changed family $X \times_{B} T$ along the section $\theta$, i.e.

$$
X_{T}^{\theta}=\mathcal{B} \ell_{\theta}\left(X \times_{B} T\right),
$$


viewed as a family of curves of genus $g-1$ with two, everywhere distinct, individually defined marked points $\theta_{x}, \theta_{y}$. We denote by $\phi$ the natural map fitting in the diagram

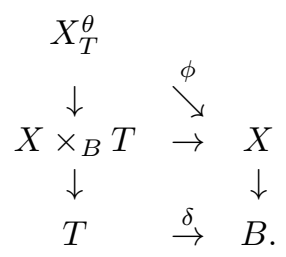

Note that a boundary datum indeed lives over the boundary of $B$; in the other direction, we can associate to any component $T_{0}$ of the boundary of $B$ a finite number boundary data in this sense: first consider a component $T_{1}$ of the normalization of $T_{0} \times_{B} \operatorname{sing}(X / B)$, which already admits a node-valued lifting $\theta_{1}$ to $X$, then further base-change by the normal cone of $\theta_{1}\left(T_{1}\right)$ in $X$ (which is 2:1 unramified, possibly disconnected, over $T_{1}$ ), to obtain a boundary datum as above. 'Typically', the curve corresponding to a general point in $T_{0}$ will have a single node $\theta$ and then the degree of $\delta$ will be 1 or 2 depending on whether the branches along $\theta$ are distinguishable in $X$ or not (they always are distinguishable if $\theta$ is a separating node and the separated subcurves have different genera). Proceeding in this way and taking all components which arise, we obtain finitely many boundary data which 'cover', in an obvious sense, the entire boundary of $B$. Such a collection, weighted so that each boundary component $T_{0}$ has total weight $=1$ is called a covering system of boundary data.

2. The tautological module. This section will provide a recursive procedure to compute arbitrary powers of the discriminant polarization $\Gamma^{(m)}$ on the Hilbert scheme $X_{B}^{[m]}$ (see $\S 1.5$, especially (1.5.17)). The computation will be a by-product of a stronger result determining the (additive) tautological module on $X_{B}^{[m]}$, to be described informally in this introduction, and defined formally in the body of the chapter (see Definition 2.42).

The tautological module, with its associated cycle map

$$
T^{m}=T_{R}^{m}(X / B) \rightarrow A^{\bullet}\left(X_{B}^{[m]}\right)_{\mathbb{Q}}
$$

is to be defined as the $A_{\mathbb{Q}}^{\bullet}(B)$-vector space generated by certain basic formal symbols called tautological classes (as described below). On the other hand, let

$$
\mathbb{Q}\left[\Gamma^{(m)}\right] \rightarrow A^{\bullet}\left(X_{B}^{[m]}\right)_{\mathbb{Q}}
$$

be the polynomial ring generated by the discriminant polarization. Then the main result of this chapter is

Theorem 2.1 (Module Theorem). Compatibly with intersection product, $T^{m}$ is $a \mathbb{Q}\left[\Gamma^{(m)}\right]$-module; moreover, the multiplication by $\Gamma^{(m)}$ can be described explicitly.

Because $1 \in T^{m}$ by definition, this statement includes the nonobvious assertion that

$$
\mathbb{Q}\left[\Gamma^{(m)}\right] \subset T^{m} ;
$$

in other words, any polynomial in $\Gamma^{(m)}$ is (explicitly) tautological. In this sense, the Theorem includes an 'explicit' (in the recursive sense, at least) computation of all the powers of $\Gamma^{(m)}$. 
Now the aforementioned basic tautological classes come in two main flavors (plus some subflavors).

i. The (classes of) (relative) diagonal loci $\Gamma_{\left(n_{1}, n_{2}, \ldots\right)}^{(m)}$ : this locus is the closure of the set of schemes of the form $n_{1} p_{1}+n_{2} p_{2}+\ldots$ where $p_{1}, p_{2} \ldots$ are distinct smooth points of the same (arbitrary) fibre.

More generally, we will consider certain 'twists' of these, denoted

$\Gamma_{\left(n_{1}, n_{2}, \ldots\right)}\left[\alpha_{1}, \alpha_{2} \ldots\right]$, where the $\alpha$. are 'base classes', i.e. cohomology classes on $X$.

ii. The node classes. First, the node scrolls $F_{j}^{n}(\theta)$ : these are, essentially, $\mathbb{P}^{1}$ bundles over an analogous diagonal locus $\Gamma_{(n .)}^{(m-n)}$ associated to a boundary family $X_{T}^{\theta}$ of $X_{B}$, whose general fibre can be naturally identified with the punctual Hilbert scheme component $C_{j}^{n}$ along the node $\theta$.

Additionally, there are the node sections: these are simply the classes $-\Gamma^{(m)} . F$ where $F$ is a node scroll as above (the terminology comes from the fact that $\Gamma^{(m)}$ restricts to $\mathcal{O}(1)$ on each fibre of a node scroll).

Finally, node scrolls and node sections define correspondence operators, pulling back (tautological) classes from a Hilbert scheme $\left(X^{\theta}\right)^{[m-n]}$.

Effectively, the task of proving Theorem 2.1 has two parts.

i. Express a product $\Gamma^{(m)} \cdot \Gamma_{(n .)}$ in terms of other diagonal loci and node scrolls, see Proporsition 2.20.

ii. For each node $\theta$ and associated ( $\theta$-normalized) boundary family $X_{T}^{\theta}$, determine a series of explicit line bundles $E_{j}^{n}(\theta), j=1, \ldots, n$ on the relative Hilbert scheme $\left(X_{T}^{\theta}\right)_{T}^{[m-n]}$ together with an identification

$$
F_{j}^{n}(\theta) \simeq \mathbb{P}\left(e_{j}^{n}(\theta) \oplus e_{j+1}^{n}(\theta)\right),
$$

such that the restriction of the discriminant polarization $-\Gamma^{(m)}$ on $F_{j}^{n}(\theta)$ becomes the standard $\mathcal{O}(1)$ polarization on the projectivized vector bundle. This is just the Node Scroll Theorem of [19]. In fact, it transpires that $e_{j}^{n}(\theta)$ is just the sum of the polarization $\Gamma^{[m-n]}$ and a suitable base divisor, that is itself a tautological class in the sense of Mumford. It then follows easily that the restriction of an arbitrary power $\left(\Gamma^{(m)}\right)^{k}$ on $F$ can be explicitly expressed in terms of tautological classes on Hilbert schemes of lower degree on boundary (hence smaller-dimensional) families (which in the stable case also have lower genus): see Theorem 2.24.

2.1. The small diagonal. We begin our study of diagonal-type loci and their intersection product with the discriminant polarization with the smallest such locus, i.e. the small diagonal. In a sense this is actually the heart of the matter, which is hardly surprising, considering as the small diagonal is in the 'most special' position vis-a-vis the discriminant. The key result is Proposition 2.5 below, which is the main ingredient in determining intersection multiplicities.

The next result is in essence a corollary to the Blowup Theorem of [19].

Let $\Gamma_{(m)} \subset X_{B}^{[m]}$ be the small diagonal, which parametrizes schemes with 1-point support, and which is the pullback of the small diagonal

$$
D_{(m)} \simeq X \subset X_{B}^{(m)}
$$

This corresponds to the distribution $\mu$ with the unique nonzero value $\mu(m)=1$. The 
restriction of the cycle map yields a birational morphism

$$
\mathfrak{c}_{m}: \Gamma_{(m)} \rightarrow X
$$

which is an isomorphism except over the nodes of $X / B$. For the remainder of the paper, we fix a covering system of boundary data $\{(T ., \delta ., \theta)$.$\} as in [19]. and focus$ on its typical node $\theta$. Thus, $\theta$ is a relative node of $X / B, \delta: T \rightarrow B$ is a generically finite surjective map onto a boundary component, and $X_{T}^{\theta}$ is the blowup of $X \times_{B} T$ in $\theta \times{ }_{B} T$. Now define a 'local model' ldeal

$$
\begin{aligned}
& J_{m}<\mathbb{C}[[x, y]], \\
& J_{m}=\left(x^{\left(\begin{array}{c}
m \\
2
\end{array}\right)}, \ldots, x^{\left(\begin{array}{c}
m-i+1 \\
2
\end{array}\right)} y^{\left(\begin{array}{c}
i \\
2
\end{array}\right)}, \ldots, y^{\left(\begin{array}{c}
m \\
2
\end{array}\right)}\right) .
\end{aligned}
$$

Because a formal neighborhood of $\theta$ in $X$ is locally a pullback of a family of the form $x y=t$, there is an analogous ideal defined in a formal neighborhood of $\theta$, and because this ideal is cosupported on $\theta$ and independent of the choice of 'local coordinates' $x, y$, it extends to an ideal

$$
J_{m}^{\theta}<\mathcal{O}_{X}
$$

Then let

$$
J_{m}^{\theta \cdot}=\bigcap_{i} J_{m}^{\theta_{i}} \subset \mathcal{O}_{X}
$$

be the ideal sheaf whose stalk at each fibre node $\theta_{i}$ is locally of type $J_{m}$. Note that $J_{m}^{\theta}$ is well-defined independent of the choice of local parameters and independent as well of the ordering of the branches at each node and invariant under permutation of the set of nodes, hence makes sense and is globally defined on $X$.

Proposition 2.2. Via $\mathfrak{c}_{m}, \Gamma_{(m)}$ is equivalent to the blow-up of $J_{m}^{\theta}$. If $\mathcal{O}_{\Gamma_{(m)}}(1)_{J}$ denotes the canonical blowup polarization, we have

$$
\mathcal{O}_{\Gamma_{(m)}}\left(-\Gamma^{(m)}\right)=\omega_{X / B}^{\otimes\left(\begin{array}{c}
m \\
2
\end{array}\right)} \otimes \mathcal{O}_{\Gamma_{(m)}}(1)_{J}
$$

Furthermore, if $X$ is smooth at a node $\theta$, then $\Gamma_{(m)}$ has multiplicity $\min (i, m-i)$ along the corresponding divisor $C_{i}^{m}-\left\{Q_{i}^{m}, Q_{i+1}^{m}\right\}$ for $i=1, \ldots, m-1$. In particular, $\Gamma_{(m)}$ is smooth along $\left(C_{1}^{m}-Q_{2}^{m}\right) \cup\left(C_{m-1}^{m}-Q_{m-1}^{m}\right)$.

Proof. We may work with the ordered versions of these objects, defined on the ordered Hilbert scheme $X_{B}^{\lceil m\rceil}$, then pass to $\mathfrak{G}_{m}$-invariants. We first work locally over a neighborhood of a point on $\theta^{m} \in X_{B}^{m}$ where $\theta$ is a fibre node. As shown in [19], $\S 6, X_{B}^{\lceil m\rceil}$ is obtained from the relative Cartesian product $X_{B}^{m}$ by a suitable blowup, namely that of the big diagonal $O D^{m}$. Because blowing up and the Hilbert scheme are both compatible with base-change, we may then assume $X$ is a smooth surface and $X / B$ is given by $x y=t$. Then the ideal of $O D^{m}$ is generated by $G_{1}, \ldots, G_{m}$ and $G_{1}$ has the Van der Monde form $v_{x}^{m}$, while the other $G_{i}$ are given by [19], $\S 6$. We try to restrict the ideal of $O D^{m}$ on the small diagonal $O D_{(m)} \simeq X$. To this end, note to begin with the natural map

$$
\mathcal{I}_{O D^{m}} \rightarrow \omega^{\left(\begin{array}{c}
m \\
2
\end{array}\right)}, \omega:=\omega_{X / B} .
$$


Indeed this map is clearly defined off the singular locus of $X_{B}^{m}$, hence by reflexivity of $\mathcal{I}_{O D^{m}}$ extends everywhere, hence moreover factors through a map

$$
\mathcal{I}_{O D^{m}} . O D_{(m)}=\mathcal{I}_{O D^{m}} \otimes \mathcal{O}_{O D_{(m)}} /(\text { torsion }) \rightarrow \omega^{\left(\begin{array}{c}
m \\
2
\end{array}\right) .}
$$

To identify the image, note that

$$
\left.\left(x_{i}-x_{j}\right)\right|_{O D_{(m)}}=d x=x \frac{d x}{x}
$$

and $\eta=\frac{d x}{x}=-\frac{d y}{y}$ is a local generator of $\omega$ along $\theta$. Therefore

$$
\left.G_{1}\right|_{O D_{(m)}}=x^{\left(\begin{array}{c}
m \\
2
\end{array}\right)} \eta^{\left(\begin{array}{c}
m \\
2
\end{array}\right)} .
$$

From [19], loc. cit.. we then deduce

$$
\left.G_{i}\right|_{\Gamma_{(m)}}=x^{\left(\begin{array}{c}
m-i+1 \\
2
\end{array}\right)} y^{\left(\begin{array}{c}
i \\
2
\end{array}\right)} \eta^{\left(\begin{array}{c}
m \\
2
\end{array}\right)}, i=1, \ldots, m .
$$

Since $G_{1}, \ldots, G_{m}$ generate the ideal $I_{O D^{m}}$ along $\theta$, it follows that over a neighborhood of $\theta$, we have

$$
I_{O D^{m}} . O D_{(m)} \simeq J_{m}^{\theta} \otimes \omega^{\left(\begin{array}{c}
m \\
2
\end{array}\right)} .
$$

This being true for each node, it is also true globally. Consequently, passing to the $\mathfrak{S}_{m}$-quotient, we also have

$$
I_{D^{m}} \cdot D_{(m)} \simeq J_{m}^{\theta} \otimes \omega^{\left(\begin{array}{c}
m \\
2
\end{array}\right)}
$$

Then pulling back to $X_{B}^{[m]}$ we get (2.1.3).

Finally, it follows from the above, plus the explicit description of the model $H_{m}$, that, along the 'finite' part $C_{i}^{m}-Q_{i+1}^{m}, \Gamma_{(m)}$ has equation $x^{m-i}-u y^{i}$ where $u$ is an affine coordinate on $C_{i}^{m}-Q_{i+1}^{m}$, from which our last assertion follows easily.

Let us now fix the node $\theta$ and analyze locally the blowup of the ideal $J_{m}=J_{m}^{\theta}=$ $\left(\ldots, x^{\left(\begin{array}{c}m-i+1 \\ 2\end{array}\right)} y^{\left(\begin{array}{c}i \\ 2\end{array}\right)}, \ldots\right)$.

LEMMA 2.3.

$$
J_{m}=\prod_{i=1}^{m-1}\left(x^{m-i}, y^{i}\right)
$$

Proof. Consider for $i=1, \ldots, m-1$ the cone $K_{i}$ in the 1 st quadrant $\mathbb{R}_{+}^{2}$ generated by $(m-i, 0)$ and $(0, i)$, i.e.

$$
K_{i}=\left(\mathbb{R}_{+}^{2}+(m-i, 0)\right) \cup\left(\mathbb{R}_{+}^{2}+(0, i)\right) .
$$

This cone corresponds to the ideal $J_{m, i}=\left(x^{m-i}, y^{i}\right)$ in the sense that $J_{m, i}$ is generated by the monomials $x^{a} y^{b}$ with $(a, b) \in K_{i}$. In a similar way, the ideal $\prod_{i} J_{m, i}$ corresponds to the cone $\sum_{i} K_{i}$. Now it is easy to see, e,g, by working with the partial sum $\sum_{i=1}^{n} K_{i}$ and using induction on $n$, that the latter cone is just equal to

$$
\left.\bigcup_{i=0}^{m}\left(\left(\begin{array}{c}
m-i+1 \\
2
\end{array}\right),\left(\begin{array}{l}
i \\
2
\end{array}\right)\right)+\mathbb{R}_{+}^{2}\right)
$$


which proves our claim.

Now let $X_{i}$ be the blowup of $X$ in $J_{m, i}=\left(x^{m-i}, y^{i}\right)$, which is the subscheme of $X \times C_{i}^{m}=X \times \mathbb{P}^{1}$ defined by

$$
x^{m-i} u_{i}=y^{i} v_{i}
$$

and contains the special points $Q_{i}^{m}=[1,0], Q_{i+1}^{m}=[0,1]$. The pullback of $J_{m, i}$ on $X_{i}$ is an invertible ideal, generated by $x^{m-i}$ near $C_{i}^{m} \backslash Q_{i+1}^{m}$ and by $y^{i}$ near $C_{i}^{m} \backslash Q_{i}^{m}$. The following is an immediate consequence of Lemma 2.3:

LEMMA 2.4. The blowup of $J_{m}$ is isomorphic to the unique component dominating $X$ of the fibre product (over $X): \prod_{i=1}^{m-1}\left(X_{i} / X\right):=X_{1} \times_{X} \ldots \times_{X} X_{m-1}$.

As was analyzed in [19], the special fibre of $\Gamma_{(m)}$, i.e. the blowup of $J_{m}$, is a chain $C_{1}^{m} \cup \ldots \cup C_{m-1}^{m}$ and the point $Q_{i}^{m} \in C_{i}^{m}$ is coupled in the cartesian product $\prod_{i=1}^{m-1} C_{i}^{m}$ with $Q_{j+1}^{m} \in C_{j}^{m}$ for $j<i$ and with $Q_{j}^{m} \in C_{j}^{m}$ for $j>i$. It follows that if we set

$$
g_{i}:=x^{\left(\begin{array}{c}
m-i \\
2
\end{array}\right)} y^{\left(\begin{array}{c}
i \\
2
\end{array}\right)} \cdot x^{m-i}=x^{\left(\begin{array}{c}
m-i+1 \\
2
\end{array}\right)} y^{\left(\begin{array}{c}
i \\
2
\end{array}\right)}
$$

then in $\Gamma_{(m)}, J_{m}$ is locally generated by by $g_{i}$ near $Q_{i}^{m}$ and by $g_{i+1}$ near $Q_{i+1}^{m}$.

Now note that the function $x$ has along $C_{i}^{m}$ multiplicity equal to the length of $\mathbb{C}[x, y] /\left(x^{m-i}-y^{i}, x\right)$, i.e. $i$; similarly, $y$ has multiplicity equal to $m-i$. Therefore, the multiplicity of the invertible ideal $J_{m}$ itself along $C_{i}^{m}$ is equal to

$$
i(m-i)+i\left(\begin{array}{c}
m-i \\
2
\end{array}\right)+(m-i)\left(\begin{array}{l}
i \\
2
\end{array}\right)=i\left(\begin{array}{c}
m-i+1 \\
2
\end{array}\right)+(m-i)\left(\begin{array}{l}
i \\
2
\end{array}\right)=\frac{i(m-i) m}{2} .
$$

Also, note that at $Q_{i+1}^{m}$, we have affine coordinates $u_{i} / v_{i}, v_{i+1} / u_{i+1}$ on $C_{i}^{m}, C_{i+1}^{m}$ respectively. These have respective zero- sets $C_{i+1}^{m}, C_{i}^{m}$ and because

$$
\left(u_{i} / v_{i}\right)\left(v_{i+1} / u_{i+1}\right)=x y
$$

which has multiplicity $m$ along either $C_{i}^{m}$ or $C_{i+1}^{m}$, it follows that $u_{i} / v_{i}$ (resp. $\left.u_{i+1} / v_{i+1}\right)$ has multiplicity $m$ along $C_{i+1}^{m}\left(\right.$ resp. $\left.C_{i}^{m}\right)$.

We summarize this discussion as follows:

Proposition 2.5. (i) The pullback ideal of $J_{m}^{\theta}$ on $\Gamma_{(m)}$ defines a Cartier divisor of the form

$$
\begin{gathered}
e_{m}^{\theta}=\sum_{i=1}^{m-1} \nu_{m, i} C_{i}^{m}(\theta), \\
\nu_{m, i}:=\frac{i(m-i) m}{2} .
\end{gathered}
$$

Moreover $x$ and $y$ have along $C_{i}^{m}(\theta)$ multiplicity equal to $m-i, i$ respectively.

(ii) Each $C_{i}^{m}$ is a $\mathbb{Q}$-Cartier divisor on $\Gamma_{(m)} ; m C_{i}^{m}$ is Cartier.

Corollary 2.6. Notations as above, we have 


$$
\begin{gathered}
(i) \quad C_{i}^{m}(\theta) C_{i+1}^{m}(\theta)=\frac{1}{m} Q_{i+1}^{m}(\theta) \\
(i i) \quad\left(C_{i}^{m}(\theta)^{2}=-\frac{1}{m} Q_{i}^{m}(\theta)-\frac{1}{m} Q_{i+1}^{m}(\theta) .\right.
\end{gathered}
$$

Proof. We will fix and suppress $\theta$.

(i)Locally at $Q_{i+1}^{m}, m C_{i}^{m}$ and $m C_{i+1}^{m}$ have respective equations $u_{i+1} / v_{i+1}, v_{i} / u_{i}$, and these locally generate an ideal of the form $\left(x^{m-i}, y^{i+1}, x y\right)$ (note $(2.1 .5)$ ), which has colength $m$.

(ii) With the above notations, the principal divisor associated to $x$ has the form $\sum_{j} j C_{j}^{m}$, therefore

$$
C_{i}^{m} \sum_{j} j C_{j}^{m}=0
$$

Using similarly the divisor of $y$ yields

$$
C_{i}^{m} \sum_{j}(m-j) C_{j}^{m}=0
$$

hence numerically,

$$
C_{i}^{m} \sum_{j} C_{j}^{m} \sim 0
$$

Because $C_{i}^{m}$ meets $C_{j}^{m}$ only for $|j-i| \leq 1$, (i) yields the result.

COROLlary 2.7. With the above notations, we have

$$
\Gamma^{(m)} \cdot \Gamma_{(m)}=\sum_{\theta, i} \nu_{m, i} C_{i}^{m}(\theta)-\left(\begin{array}{c}
m \\
2
\end{array}\right)\left[\omega_{X / B}\right] .
$$

Moreover, if $\operatorname{dim}(B)=1$, we have:

$$
e_{m}^{2}=-\sigma \nu_{m}, \nu_{m}:=\frac{m^{2}(m-1)(m+1)}{12},
$$

where $\sigma$ is the number of nodes of $X / B$;

$$
\int_{\Gamma_{(m)}}\left(\Gamma^{(m)}\right)^{2}=-\sigma \nu_{m}+\left(\begin{array}{c}
m \\
2
\end{array}\right)^{2} \omega_{X / B}^{2} .
$$

REMARK 2.8. The components $C_{i}^{m}(\theta), i=1, \ldots, m-1$ of $e_{m}$ are $\mathbb{P}^{1}$-bundles over the normalization $B(\theta)$ of the boundary component corresponding to the node $\theta$. These are special cases of the node scrolls, encountered in the previous section, which will be further discussed in $\S 2.4$ below. See $\S 3.3$ for further discussion of the small diagonal and its intersection theory.

For the remainder of the paper, we set

$$
\omega=\omega_{X / B}
$$

We will view this interchangeably as line bundle or divisor class. 
2.2. Monoblock and polyblock digaonals: Ordered case. Returning to our family $X / B$ of nodal curves, we now begin extending the results of $\S 2.1$ to the more general diagonal loci as defined above, first for those that live over all of $B$, and subsequently for loci associated to the boundary. In this section, We will work with ordered objects, chiefly as a tool for understanding their unordered analogues, to be considered in the next section.

We will work here with the ordered relative Hilbert scheme of the nodal family $X / B$, defined as

$$
X_{B}^{\lceil m\rceil}=X_{B}^{[m]} \times_{X_{B}^{(m)}} X_{B}^{m}
$$

where $X_{B}^{[m]} \rightarrow X_{B}^{(m)}$ is of course the cycle map $c_{m}$ studied at length in [19]. As discussed in 1.5, $X_{B}^{[m]}$ is endowed with the (half) discriminant $\Gamma^{(m)}$. We denote by $\Gamma^{\lceil m\rceil}$ the pullback of the latter on $X_{B}^{\lceil m\rceil}$, which is effective, reduced and Cartier and admits a splitting as Weil divisor

$$
\Gamma^{\lceil m\rceil}=\sum_{1 \leq a<b \leq m} D_{a, b}
$$

where the summands are pullbacks of diagonals in the 2-fold product and are not Cartier.

We recall the ordered polyblock diagonal loci $O D_{(I .)}=O D_{(I .), X / B}$ discussed in $\S 1.2$. Here we will use this notation to refer to the appropriate loci in the relative Cartesian product $X_{B}^{m}$. In particular, we have the ordered monoblock diagonal

$$
O D_{I, X / B}^{m}=O D_{I}=\subset X_{B}^{m}
$$

and the big diagonal

$$
O D^{m}=\sum_{1 \leq a<b \leq m} O D_{a, b}^{m}
$$

Similar loci exist in the ordered Hilbert scheme:

$$
\Gamma_{I}=\Gamma_{I}^{\lceil m\rceil}:=o c^{-1}\left(O D_{I}\right) \subset X_{B}^{\lceil m\rceil} .
$$

Note that $O D_{I}$, hence $\Gamma_{I}$, are defined locally near a node by equations

$$
x_{i}-x_{j}=0=y_{i}-y_{j}, \forall i, j \in I .
$$

Generally, for any b-partition

$$
(I .)=\left(I_{1}, \ldots, I_{r}\right) \subset[1, m]
$$

we have an analogous locus (ordered polyblock diagonal)

$$
\Gamma_{I_{1}|\ldots| I_{r}}=\Gamma_{I_{1}|\ldots| I_{r}}^{\lceil m\rceil} \subset X_{B}^{\lceil m\rceil}
$$

and note that

$$
\Gamma_{I_{1}|\ldots| I_{r}}=\Gamma_{I_{1}} \cap \ldots \cap \Gamma_{I_{r}}
$$


(transverse intersection). Recall that $r$ is called the length of the b-partition $(I$.$) and$ denoted $\ell(I$.). Also

$$
\Gamma_{(I .)}=o c^{-1}\left(O D_{(I .)}\right)
$$

where $O D_{(I .)} \subset X_{B}^{m}$ is the analogous polyblock diagonal. We may view $\Gamma_{(I .)}$ as an operator

$$
\begin{gathered}
\Gamma_{(I .)}[]: \bigotimes^{r} R \rightarrow H_{\bullet}\left(X_{B}^{\lceil m\rceil}\right), r=\ell(I .) \\
(\alpha .) \mapsto \Gamma_{(I .)} \cap o c^{*}(\alpha .)
\end{gathered}
$$

where as usual $H_{\bullet}$ denotes a homology theory coarser than Chow. Thus, the values of $\Gamma_{(I .)}[]$ are homology rather than cohomology classes. However, their $\mathfrak{S}_{m^{-}}$ symmetrized versions will descend to the (unordered) Hilbert scheme $X_{B}^{[m]}$, which is typically smooth, so the distinction between homology and cohomology will not matter.

Now our first goal is to determine the intersection action of discriminant operator on a monoblock diagonal cycle, i.e. to determine the intersection cycle $\Gamma^{\lceil m\rceil} \cdot \Gamma_{I}$. In this computation, a key technical question is to determine the part of $O D_{I}$ and $\Gamma_{I}$ over the boundary of $B$, or at least its irreducible components. Thus for each boundary datum $(\theta, T, \delta)$, with the associated map $\phi: X_{T}^{\theta} \rightarrow X$ and cartesian power

$$
\phi^{m}:\left(X^{\theta}\right)_{T}^{m} \rightarrow X_{B}^{m},
$$

we need to determine $\left(\phi^{m}\right)^{*}\left(O D_{I}\right)$ and its inverse image in $\left(X_{T}^{\theta}\right)^{\lceil m\rceil}$ which we call the $(\theta, T, \delta)$ boundary of $\Gamma_{I}$. A priori, it is clear that any difference between the answers in Sym and Hilb will have to do with node-supported loci, i.,e. node scrolls.

To state the answer, we recall from [19] the ordered node scroll $O F_{j}^{I}$, which is the portion of $\varpi^{-1}\left(F_{j}^{n}(\theta), n=|I|\right.$, where the $n$ points coalesced in $\theta$ lie in the $I$ indexed coordinates. This maps to $\left(X_{T}^{\theta}\right)^{\lceil[1, m] \backslash I\rceil}$ (i.e. a copy of $\left(X_{T}^{\theta}\right)^{\lceil m-n\rceil}$ indexed by $\{1, \ldots, m\} \backslash I$,rather than $\{1, \ldots, m-n\})$. Locally near $\theta_{x} \cup \theta_{y},\left(X_{T}^{\theta}\right)^{\lceil[1, m] \backslash I\rceil}$ breaks up into branches corresponding to decompositions $\{1, \ldots, m\} \backslash I=K_{x} \amalg K_{y}$, where $K_{x}, K_{y}$ are the indices of the points which lie in the $x$ or $y$-branches, denoted $X^{\prime}, X$ " respectively. We denote the corresponding branches of $O F_{j}^{I}$ by $O F_{j}^{I: K_{x} \mid K_{y}}(\theta)$.

For index-sets $I \subset K$, we will use the notation $K / I$ to denote the quotient set identifying $I$ to a singleton, i.e. $(K \backslash I) \bigsqcup\{I\}$. Correspondingly, $X^{K / I}$ will denote the subset of $X^{K}$ consisting of points whose components indexed by $I$ are mutually equal.

We begin with a key technical Lemma analyzing the boundary of the monoblock diagonal $\Gamma_{I}$.

Lemma 2.9. Set-theoretically, the $(\theta, T, \delta)$ boundary of $\Gamma_{I}$ is the union of the following loci, each one itself a union of irreducible components of the boundary:

i. for each index-set $K,[1, m] \supset K \supset I$, a locus $\tilde{\Theta}_{K / I}$, mapping birationally to its image $\Theta_{K / I} \subset O D_{I}=\left(X^{\prime}\right)^{K / I} \times\left(X^{\prime \prime}\right)^{K^{c}}$;

ii. for each $K \subset I^{c}=[1, m] \backslash I$, a locus $\tilde{\Theta}_{K / I}$, mapping birationally to its image $\Theta_{K / I} \subset O D_{I}=\left(X^{\prime}\right)^{K} \times\left(X^{\prime \prime}\right)^{K^{c} / I}$; 
iii. for each $K$ straddling $I$ and $I^{c}$ (i.e. meeting both), and each $j=1, \ldots,|I|-1$, a component $O F_{j}^{I: K-I \mid K^{c}-I}(\theta) \subset O F_{j}^{I}(\theta)$ projecting as $\mathbb{P}^{1}$-bundle to its image in $\left(X_{T}^{\theta}\right)^{\lceil m-|I|\rceil}$, which lies over $\left(X^{\prime}\right)^{K-I} \times_{T}\left(X^{\prime \prime}\right)^{K^{c}-I}=:\left(X_{T}^{\theta}\right)^{K-I \mid K^{c}-I} \subset$ $\left(X_{T}^{\theta}\right)^{m-|I|}$.

Proof. The loci of type (i), (ii) are clearly there and any other component must occur at the boundary. Hence, we may fix a node $\theta$ and work locally over a neighborhood of $\theta$ in $X$. The main point is first to determine the boundary of $O D_{I}$ (in the symmetric product). But this is easily determined as in the $\Theta$ decomposition of [19] $\S 4$ : the boundary is given locally by

$$
\bigcup_{K \subset[1, m]} O D_{I} \cap \Theta_{K}
$$

Set $\Theta_{K / I}=O D_{I} \cap \Theta_{K}$. To describe these, there are 3 cases depending on $K$ :

i. if $I \subset K$, then

$$
\Theta_{K / I}=\left(X^{\prime}\right)^{K / I} \times\left(X^{\prime \prime}\right)^{K^{c}}
$$

ii. if $I \subset K^{c}$, then

$$
\Theta_{K / I}=\left(X^{\prime}\right)^{K} \times\left(X^{\prime \prime}\right)^{K^{c} / I}
$$

iii. otherwise, i.e. if $I$ meets both $K$ and $K^{c}$, then

$$
\begin{gathered}
\Theta_{K / I}=\left\{y_{i}=0, \forall i \in K \cup I, x_{i}=0, \forall i \in K^{c} \cup I\right\} \\
=\left(X^{\prime}\right)^{K-I} \times\left(X^{\prime \prime}\right)^{K^{c}-I} \times 0^{I}=: X^{K-I \mid K^{c}-I} .
\end{gathered}
$$

Now is an elementary check that the loci of type (i) and (ii) are precisely the irreducible components of the special fibre of $O D_{I}$, while the union of the loci $\Theta_{K / I}$ of type (iii) coincides with the intersection of $O D_{I}$ with the fundamental locus (=image of exceptional locus) of the ordered cycle map $o c_{m}$, i.e. the locus of cycles containing the node with multiplicity $>1$. Also, each $\Theta_{K / I}$ of type (iii) is of codimension 2 in $O D_{I}$. On the other hand, each such $\Theta_{K / I}=X^{K-I \mid K^{c}-I}$ is just a component of the inverse image in $X_{B}^{m}$ of the locus denoted $X^{(a, b)}$ in [19], $\S 5$, where $a=|K-I|, b=$ $\left|K^{c}-I\right|$, and therefore by that Lemma, the ordered cycle map over it is a union of $\mathbb{P}^{1}$ bundles, viz

$$
o c_{m}^{-1}\left(X^{K-I \mid K^{c}-I}\right)=\bigcup_{j=1}^{|I|-1} O F_{j}^{I: K-I \mid K^{c}-I}
$$

where $O F_{j}^{I: K-I \mid K^{c}-I}$ is the pullback of $F_{j}^{(m-a-b: a \mid b)}$ over $X^{K-I \mid K^{c}-I}$, which is a $\mathbb{P}^{1}$ bundle with fibre $C_{j}^{|I|}$. This concludes the proof.

Notice that, given disjoint index-sets $K_{1}, K_{2}$ with $K_{1} \coprod K_{2}=I^{c}$, the number of straddler sets $K$ such that $K-I=K_{1}, K^{c}-I=K_{2}$ is precisely $2^{n}-2$ (i.e. the number of proper nonempty subsets of $I$ ). Thus, a given $O F_{j}^{I: K_{1} \mid K_{2}}$ will lie on this many components of $\tilde{\Theta}$. This however is a completely separate issue from the 
multiplicity of $O F_{j}^{I: K_{1} \mid K_{2}}$ in the intersection cycle $\Gamma^{[m]}{ } \Gamma_{I}$, which has to do with the blowup structure and will be determined below.

From the foregoing analysis, we can easily compute the intersection of a monoblock diagonal cycle with the discriminant polarization, as follows. We will fix a covering system of boundary data $\left(T_{s}, \delta_{s}, \theta_{s}\right)$,and recall that each datum must be weighted by $\frac{1}{\operatorname{deg}\left(\delta_{s}\right)}$ (cf. $\S 1.7$.

Proposition 2.10. We have an equality of divisor classes on $\Gamma_{I}$ :

$$
\begin{gathered}
\Gamma^{\lceil m\rceil} . \Gamma_{I}=\sum_{i<j \notin I} \Gamma_{I \mid\{i, j\}}+|I| \sum_{i \notin I} \Gamma_{I \cup\{i\}}-\left(\begin{array}{c}
|I| \\
2
\end{array}\right) p_{\min (I)}^{*} \omega \\
+\sum_{s} \frac{1}{\operatorname{deg}\left(\delta_{s}\right)} \sum_{j=1}^{|I|-1} \nu_{|I|, j} \delta_{s, j *}^{I} O F_{j}^{I}\left(\theta_{s}\right),
\end{gathered}
$$

where $I \mid\{i, j\}$ and $I \cup\{i\}$ denote the evident diblock partition and uniblock, respectively, the 4 th term denotes the class of the image of the node scroll on $\Gamma_{I}, O F_{j}^{I}(\theta)=$

$\sum_{K_{2}=I^{c}} O F_{j}^{I: K_{1} \mid K_{2}}(\theta), \delta_{s, j}^{I}$ is the natural map of the latter to $\Gamma_{I} \subset X_{B}^{[m]}$ and the multiplicities $\nu_{n, j}$ are given by (2.1.6) ; precisely put, the line bundle on $\Gamma_{I}$ given by $\mathcal{O}_{\Gamma_{I}}\left(\Gamma^{\lceil m\rceil}\right) \otimes p_{\min (I)}^{*}\left(\omega^{|I|}\right)$ is represented by an effective divisor comprising the 1st, 2nd and 4 th terms of the RHS of (2.2.9).

Proof. To begin with, the asserted equality trivially holds away from the exceptional locus of $o c_{m}$, where the 1st, second and third summands come from components $\Gamma_{i, j}$ of $\Gamma^{\lceil m\rceil}$ having $|I \cap\{i, j\}|=0,1,2$, respectively.

Next, both sides being divisors on $\Gamma_{I}$, it will suffice to check equality away from codimension 2, e.g. over a generic point of each (boundary) locus $\left(X_{T}^{\theta}\right)^{K-I \mid K^{c}-I}$. But there, our cycle map $o c_{m}$ is locally just $o c_{r} \times$ iso, $r=|I|$, with

$$
\Gamma^{\lceil m\rceil} \sim \Gamma^{\lceil r\rceil}+\sum_{\{i, j\} \not \subset I} \Gamma_{i, j} .
$$

We are then reduced to the case of the small diagonal, discussed in $§ 2.1$, especially Proposition 2.5.

Now this result immediately implies an analogous one for the operator

$$
\Gamma_{I}[]: \bigotimes^{m-|I|+1} R \rightarrow A_{\bullet}\left(X_{B}^{[m]}\right),
$$

whose arguments, as products of (co)homology classes, can be represented by cycles in generic position. Recall that by convention, the first $R$ factor is associated with the $I$ block. Also, as $\Gamma^{\lceil m\rceil}$ is Cartier, it defines an endomorphism

$$
\Gamma^{\lceil m\rceil}=. \cup\left[\Gamma^{\lceil m\rceil}\right]: A_{\bullet}\left(X_{B}^{\lceil m\rceil}\right) \circlearrowleft .
$$

The result can be written compactly using the 'formal discriminant' operator' of $\S 1.4$, as follows.

Corollary 2.11. Notations as above,

$$
\begin{aligned}
\Gamma^{\lceil m\rceil} . \Gamma_{I}[\alpha .]= & \Gamma_{I}\left[\operatorname{ODsc}(\alpha .)-O U_{\omega}(\alpha .)\right] \\
& +\sum_{s} \frac{1}{\operatorname{deg}\left(\delta_{s}\right)} \sum_{j=1}^{|I|-1} \nu_{|I|, j} \delta_{s, j *}^{I} O F_{j}^{I}\left(\theta_{s}\right)\left[o u_{I, g}(\alpha .)\right], \alpha . \in \operatorname{TS}_{I}(R)
\end{aligned}
$$


where ou $u_{I, g}$ is as in (1.4.10) with $g(\alpha)=\theta_{s}^{*}(\alpha)$, where $\theta_{s}$ is viewed as a (partial) section $T_{s} \rightarrow X$.

We note that in the node scroll operator above, we are viewing $H \cdot(B) \subset H^{\cdot}\left(X^{\theta_{s}}\right)$ via pullback through $X^{\theta_{s}} \rightarrow T_{s} \rightarrow B$.

Our next goal is to extend the foregoing result from the monoblock to the polyblock case- still in the ordered setting. While the extension in question is in principle straightforward, it is a bit complicated to describe. Again, a key issue is to describe the boundary of a polyblock diagonal locus $O D_{(I .)}$ in terms of the $\Theta$ decomposition of [19], §4.. Fix a boundary datum $(T, \delta, \theta)$. To simplify notations, we will assume, losing no generality, that the partition $I$. is full, i.e. $\bigcup I_{j}=[1, m]$. Now consider an index-set $K \subset[1, m]$. As before, $K$ is said to be a straddler with respect to a block $I_{\ell}$ of $(I$.$) , and I_{\ell}$ is a straddler block for $K$, if $I_{\ell}$ meets both $K$ and $K^{c}$. The straddler number $\operatorname{strad}_{(I .)}(K)$ of $K$ w.r.t. $(I$.$) is the number of straddler blocks I_{\ell}$. The straddler portion of $(I$.) relative to $K$ is by definition the union of all straddler blocks, i.e.

$$
s_{K}(I .)=\bigcup_{I_{\ell} \cap K \neq \emptyset \neq I_{\ell} \cap K^{c}} I_{\ell} .
$$

The $x$ - (resp. $y$-)-portion of (I.) (relative to $K$, of course) are by definition the partitions

$$
x_{K}(I .)=\left\{I_{\ell}: I_{\ell} \subset K\right\}, y_{K}(I .)=\left\{I_{\ell}: I_{\ell} \subset K^{c}\right\} .
$$

Finally the multipartition data associated to $(I$.) w.r.t. $K$ are

$$
\Phi_{K}(I .)=\left(s_{K}(I .): x_{K}(I .) \mid y_{K}(I .)\right) .
$$

In reality, this is a partition broken up into 3 parts: the nodebound part $s_{K}(I$.$) , a$ single block, plus 2 at large parts, an $x$ part and a $y$ part. As before, we set

$$
X^{\Phi_{K}(I .)}=\left(X^{\prime}\right)^{x_{K}(I .)} \times\left(X^{\prime \prime}\right)^{y_{K}(I .)}
$$

and equip it as before with the map to $X_{s}^{m}$ obtained by inserting the node $\theta$ at the $s_{K}(I$.$) positions. Now the analogue of Lemma 2.9$ is the following

Lemma 2.12. For any partition (I.) and boundary datum $(T, \delta, \theta)$, the corresponding boundary portion of $\Gamma_{(I .)}$ is

$$
\bigcup_{\operatorname{strad}_{(I .)}(K)=0} \tilde{\Theta}_{K,(I .)} \cup \bigcup_{\ell} \bigcup_{I^{\prime} \cdot \amalg} \bigcup_{I^{\prime \prime} .=I . \backslash I_{\ell}} \bigcup_{j=1}^{\left|I_{\ell}\right|-1} O F_{j}^{\left(I_{\ell}: I^{\prime} \cdot \mid I^{\prime \prime} \cdot\right)}(\theta) .
$$

Proof. First, one easily verifies:

$$
O D_{(I .)} \cap \Phi_{K}=X^{\Phi_{K}(I .)}=: \Theta_{K,(I .)}
$$

so that

$$
O D_{(I .)} \cap\left(X^{\theta}\right)_{T}^{m}=\bigcup_{K \subset[1, m]} \Theta_{K,(I .)} .
$$


Now, an elementary observation is in order. Clearly, the codimension of $O D_{(I .)}$ in $X_{B}^{m}$ is $\sum_{\ell}\left(\left|I_{\ell}\right|-1\right)$, and this also equals the codimension of $O D_{(I .)} \cap\left(X^{\theta}\right)_{T}^{m}$ in $\left(X^{\theta}\right)_{T}^{m}$.

On the other hand, we have

$$
\begin{aligned}
\operatorname{dim}\left(\Theta_{K,(I .)}\right) & =m-\left(\sum_{I_{\ell} \text { nonstraddler rel } K}\left(\left|I_{\ell}\right|-1\right)+\sum_{I_{\ell} \text { straddler rel } K}\left|I_{\ell}\right|\right) \\
& =m-\sum_{\ell}\left(\left|I_{\ell}\right|-1\right)-\operatorname{strad}_{(I .)}(K) .
\end{aligned}
$$

It follows that

- the index-sets $K$ such that $\Theta_{K,(I .)}$ is a component of the boundary $O D_{(I .)} \cap$ $\left(X_{T}^{\theta}\right)^{m}$ are precisely the nonstraddlers;

- those $K$ such that $\Theta_{K,(I .)}$ is of codimension 1 in the special fibre are precisely those of straddle number 1 (unistraddlers).

Next, what are the preimages of these loci upstairs in the ordered Hilbert scheme $X_{B}^{\lceil m\rceil}$ ? They can be analyzed as in the monoblock case:

- if $K$ is a nonstraddler, a general cycle parametrized by $\Theta_{K,(I .)}$ is disjoint from the node, so there will be a unique component $\tilde{\Theta}_{K,(I .)} \subset o c_{m}^{-1}\left(\Theta_{K,(I .)}\right.$ dominating $\Theta_{K,(I .)}$;

- if $K$ is a unistraddler (straddle number $=1$ ), the dominant components of $o c_{m}^{-1}\left(\Theta_{K,(I .)}\right)$ will be the $\mathbb{P}^{1}$-bundles $F_{j}^{\Phi_{K}(I .)}, j=1,, s_{K}(I)-$.1 ; note that if $I_{\ell}$ the unique block making $K$ a straddler, then $\Phi_{K}(I)=.\left(I_{\ell}: x_{K}(I) \mid. y_{K}(I).\right)$; moreover as $K$ runs through all unistraddlers, $\Phi_{K}(I$.) runs through the date consisting of a choice of block $I_{\ell}$ plus a partition of the set of remaining blocks in two (' $x$ - and $y$-blocks');

- because all fibres of $o c_{m}$ are at most 1-dimensional, while every component of the boundary is of codimension 1 in $\Gamma_{(I .)}$, no index-set $K$ with straddle $\operatorname{number}_{\operatorname{strad}}^{(I .)}(K)>1$ (i.e. multistraddler) can contribute a component to that special fibre.

This completes the proof.

The import of the Lemma is that the analysis leading to Proposition 2.10 extends with no essential changes to the polyblock case, and therefore the natural analogue of that Proposition holds. This is the subject of the next Corollary which for convenience will be stated in operator form. The statement is nearly identical to the monoblock case, except that the node scrolls appearing will themselves contain a polydiagonal conditions on the variable points on $X^{\theta}$. We will write $\Gamma_{(I .), Y}$ to indicate the appropriate polydiagonal locus associated to a given family $Y$ (e.g. $Y=X / B, X_{T}^{\theta} / T$ etc.) then define

$$
O F_{j}^{I_{\ell} / I \cdot}(\theta)=O F_{j}^{I_{\ell}} \cdot \Gamma_{(I .) \backslash I_{\ell}, X_{T}^{\theta} / T} \subset\left(X_{T}^{\theta}\right)^{\left\lceil[1, m] \backslash I_{\ell}\right\rceil} .
$$

In words, this is the pullback of the appropriate polyblock diagonal from the base of the (ordered) node scroll. As above, the pullback via $X_{T}^{\theta} \rightarrow X$ gives an inclusion $H \cdot(X) \rightarrow H \cdot\left(X_{T}^{\theta}\right)$ so for any subring $R \subset H \cdot(X)$ containing $\omega$ the operator

$$
O F_{j}^{I_{\ell} / I \cdot}(\theta)[]: \bigotimes^{m-\left|I_{\ell}\right|+1} R \rightarrow H \cdot\left(X_{B}^{\lceil m\rceil}\right)
$$

is defined. 
CoRollary 2.13. For any block partition $I .=I_{1}|\ldots| I_{r}$ on $[1, m]$, we have an equality of operators

$$
\begin{aligned}
\Gamma^{[m]} \circ \Gamma_{(I .)}[]= & \Gamma_{(I .)}[] \circ\left(\mathrm{ODsc}-O U_{\omega}\right) \\
& +\sum_{s} \frac{1}{\operatorname{deg}\left(\delta_{s}\right)} \sum_{\ell=1}^{r} \sum_{j=1}^{\left|I_{\ell}\right|-1} \nu_{\left|I_{\ell}\right|, j} \delta_{s, j *}^{I_{\ell}} O F_{j}^{I_{\ell} / I .}\left(\theta_{s}\right)[] \circ o u_{\ell, \theta_{s}^{*}}
\end{aligned}
$$

where $u_{\ell, \theta_{s}^{*}}$ is interior multiplication by $\theta_{s}^{*}: R \rightarrow H_{\bullet}(B)$ as in (1.4.10).

What the Corollary means is that the discriminant action on the ordered Hilbert scheme is the 'classical' action on the Cartesian product (cf. Lemma 1.2), plus a boundary term as above. In the next section we will derive (easily) and explore the corresponding result for the (unordered) Hilbert scheme.

2.3. Monoblock and polyblock diagonals: Unordered case. Here we will transport the formulae of the last section to the (unordered) Hilbert scheme. This is essentially straightforward, and is generally accomplished by applying to the appropriate ordered formulae push-forward by the symmetrization map

$$
\varpi_{m}: X_{B}^{\lceil m\rceil} \rightarrow X_{B}^{[m]} .
$$

We begin with the monoblock case. Recall first the the monoblock (unordered) diagonal operator $\Gamma_{(n)}[]$ which may be defined for $n>1$ as

$$
\Gamma_{(n)}[\alpha .]=\frac{1}{(m-n) !} \varpi_{m *}\left(\Gamma_{(I)}\right)[\alpha .], \alpha .=\alpha_{1} \otimes\left(\alpha_{2} \ldots \alpha_{m-n}\right) \in R \otimes \operatorname{Sym}_{\mathbb{Q}}^{m-n}(R) .
$$

Thus $\alpha_{1}$ is associated to a block of size $n$ while each of $\alpha_{2}, \ldots, \alpha_{m-n}$ is associated to a singleton block. Generally for a distribution $\mu$ the polyblock diagonal operators $\Gamma_{\mu}$ [] can be defined similarly by

$$
\Gamma_{\mu}[]=\frac{1}{a(\mu)} \varpi_{*} \Gamma_{(I .)}[]: \bigotimes_{n} \operatorname{Sym}^{\mu(n)}(R)=\operatorname{TS}_{\mu}(R) \rightarrow A_{\bullet}\left(X_{B}^{[m]}\right)
$$

where (I.) is any b-partition with distribution $\mu$ and $a(\mu)=\prod_{n} \mu(n)$ ! is the degree of the restricted symmetrization map $\Gamma_{(I .)} \rightarrow \Gamma_{\mu}$ and $\operatorname{Sym}^{\mu(n)}(R)$ is viewed as subring

of the tensor product $\stackrel{\mu(n)}{\bigotimes} R$. We will often specify a distribution by specifying only its non-singleton blocks. Thus $\Gamma_{(n), m}$ or $\Gamma(n)$ for $n>1$ is short for $\Gamma_{\mu}$ with $\mu$ of weight $m$ with $\mu(n)=1, \mu(1)=m-n$; similarly for $\Gamma_{\left(n \mid n^{\prime} \ldots\right)}$. Note that in the case of the trivial partition $\left(1^{m}\right)$, the corresponding operator

$$
\Gamma_{\left(1^{m}\right)}[]: \operatorname{Sym}^{m}(R) \rightarrow A_{\bullet}\left(X^{[m]}\right)
$$

is just pullback by the cycle map $c_{m}$. This map admits 'transpose' (trace map)

$$
c_{m *}: A_{\bullet}\left(X^{[m]}\right) \rightarrow \operatorname{Sym}^{m}(R) .
$$

Also, corresponding to the Cartier divisor $\Gamma^{(m)}$, we have the endomorphism

$$
\Gamma^{(m)}=. \cup\left[\Gamma^{(m)}\right]: A_{\bullet}\left(X_{B}^{[m]}\right) \circlearrowleft .
$$


Note that

$$
\Gamma^{(m)} \Gamma_{\left(1^{m}\right)}[]=\frac{1}{2} \Gamma_{\left(21^{m-2}\right)}=\frac{1}{2} \Gamma_{(2)}[]
$$

We will use $\Gamma_{\mu} \in A_{\bullet}\left(X_{B}^{[m]}\right)$ to denote $\Gamma_{\mu}\left[1_{\mu}\right]$ where $1_{\mu} \in \operatorname{TS}_{\mu}(R)$ is the uniquely determined product of $1_{R}$ factors. We will also use $\Gamma_{\mu}[(\alpha)$.$] to denote the operator$

$$
\begin{gathered}
\operatorname{TS}_{\mu}(R) \rightarrow A_{\bullet}\left(X_{B}^{[m]}\right), \\
(\beta .) \mapsto \Gamma_{\mu}\left[\left(\alpha \cdot \cdot_{R} \beta .\right)\right]
\end{gathered}
$$

where the product refers to the product in $\mathrm{TS}_{\mu}(R)$ induced by the product in $R$. Taken together, the various $\Gamma_{\mu}[]$ operators can be assembled to a single operator

$$
\Gamma \bullet m: \operatorname{TS}(R) \rightarrow A_{\bullet}\left(X_{B}^{[m]}\right) .
$$

The main result of this section, Theorem 2.23 expresses the action of the discriminant on $\Gamma \bullet m$.

REMARK 2.14. Using the Nakajima-inspired notation introduced in [21], we can write

$$
\begin{aligned}
\Gamma_{(n)}[\alpha] & =q_{n}[\alpha], \\
\Gamma_{\mu}[\alpha .] & =\prod_{n} \star\left(q_{n}\left[\alpha_{n, 1}\right] \star \ldots \star q_{n}\left[\alpha_{n, \mu(n)}\right]\right) .
\end{aligned}
$$

Here all products are the 'external' or star products (see 1.3) and $q_{n}[\alpha]=\Gamma_{(n)}[\alpha]$ is the Nakajima-like 'creation' operator (evaluated on 1).

Note the following elementary facts:

i.

$$
\varpi_{m *}\left(\Gamma^{\lceil m\rceil} \cdot \Gamma_{I}\right)=\Gamma^{(m)} \cdot \varpi_{m *} \Gamma_{I}
$$

(projection formula, because $\varpi_{m}^{*}\left(\Gamma^{(m)}\right)=\Gamma^{\lceil m\rceil}$; NB $\varpi$ is ramified over the support of $\Gamma^{(m)}$, still no factor of 2 in $\varpi_{m}^{*}\left(\Gamma^{(m)}\right)$, by our definition of $\Gamma^{(m)}$ as ii. $1 / 2$ times its support);

$$
\varpi_{m *}\left(\Gamma_{I}[\alpha]\right)=(m-n) ! \Gamma_{(n)}[\alpha], n=|I|>1
$$

iii.

$$
\varpi_{m *}\left(\Gamma_{I \mid\{i, j\}}\right)=\left\{\begin{array}{l}
(m-n-2) ! \Gamma_{(n \mid 2)}, \quad n \neq 2 ; \\
2(m-n-2) ! \Gamma_{(2 \mid 2)}, \quad n=2, \\
\left(1+\delta_{2, n}\right)(m-n-2) ! \Gamma_{(n \mid 2)}, \quad \forall n
\end{array}\right.
$$

( $\delta=$ Kronecker delta) here $\Gamma_{(n \mid 2)}$ is the diagonal locus corresponding to the distribution (of weight $m$ ) with blocks of sizes $n, 2$ plus singletons; iv.

$$
\varpi_{m *}\left(\Gamma_{I \amalg\{i\}}\right)=(m-n-1) ! \Gamma_{(n+1)} ;
$$


v.

$$
\begin{aligned}
& \varpi_{m *}\left(O F_{j}^{I: K-I \mid K^{c}-I}(\theta)\right)=a ! b ! F_{j}^{(n: a \mid b)}(\theta), \\
& a=|K-I|, b=\left|K^{c}-I\right|=m-n-a
\end{aligned}
$$

where we recall that $F_{j}^{(n: a \mid b)}(\theta)$ is the unordered analogue of the node scroll $F_{j}^{\left(I: K^{\prime} \mid K^{\prime \prime}\right)}$; moreover the number of distinct subsets $K-I$ with $a=|K-I|$, for fixed $I$ and $a$, is $\left(\begin{array}{c}m-n \\ a\end{array}\right)$ which easily implies that the push-forward, properly weighted, of the total of the ordered node scrolls by symmetrization equals the total of the unordered node scrolls.

Putting these together with Proposition 2.10, we conclude

$$
\begin{aligned}
\Gamma^{(m)} . \Gamma_{(n)} \sim & \frac{1+\delta_{2, n}}{2} \Gamma_{(n \mid 2)}+n \Gamma_{(n+1)}-\left(\begin{array}{c}
n \\
2
\end{array}\right) \Gamma_{(n)}[\omega] \\
& +\sum_{s} \frac{1}{\operatorname{deg}\left(\delta_{s}\right)} \sum_{a=0}^{m-n} \sum_{j=1}^{n-1} \nu_{n, j} \delta_{s, j *}^{n} F_{j}^{(n: a \mid m-n-a)}\left(\theta_{s}\right)
\end{aligned}
$$

Here and elsewhere, $\Gamma_{(n)}[\omega]$ is short for $\Gamma_{(n)}\left[\omega \otimes 1^{m-n}\right], \omega \otimes 1^{m-n} \in R \otimes \operatorname{Sym}_{\mathbb{Q}}^{m-n}(R)$, either as cycle or operator.

Set

$$
\sum_{a=0}^{m-n} F_{j}^{(n: a \mid m-n-a)}=F_{j}^{n, m}
$$

(when $m$ is understood, we will denote this by $F_{j}^{n}$ ). We note that in this sum, the first 3 terms in (2.3.8) match up exactly with (1.4.11), where the first term corresponds to uniting two singleton blocks and the second to uniting a singleton block with the $n$-block. Therefore the formula may be extended to the twisted case and written more compactly as follows

Proposition 2.15. For any monoblock diagonal $\Gamma_{(n)}, n>1$, we have

$$
\Gamma^{(m)} . \Gamma_{(n)}[] \sim \Gamma_{(n)} \circ\left(\operatorname{Dsc}^{(m)}-U_{\omega}\right)+\sum_{s} \frac{1}{\operatorname{deg}\left(\delta_{s}\right)} \sum_{j=1}^{n-1} \nu_{n, j} \delta_{s, j *}^{n} F_{j}^{n, m}\left(\theta_{s}\right)[] \circ u_{n, \theta_{s}^{*},(n)} .
$$

where $u_{n, \theta_{s}^{*},(n)}$ is as in (1.4.9) and $\nu_{n, j}=\frac{1}{2} j(n-j) n$.

For simplicity of notation, we will denote $u_{n, g,(n)}$ by $u_{n, g}$ (e.g. $u_{n, \theta^{*}}$ ).

When $n=2, \Gamma_{(n)}$ is just $2 \Gamma^{(m)} \Gamma_{\left(1^{m}\right)}$, hence (cf. (1.4.7))

Corollary 2.16.

$$
\begin{aligned}
\left(\Gamma^{(m)}\right)^{2} \Gamma_{\left(1^{m}\right)[]}= & \frac{1}{2} \Gamma_{(2 \rightarrow 2)}[] \circ u_{\left(\left(1^{2}\right) ;\left(1^{2}\right):\left(1^{m}\right)\right)}+\Gamma_{(3)}[] \circ u_{\left(\left(1^{3}\right):\left(1^{m}\right)\right)}-\Gamma^{(m)}[\omega] \\
& +\sum_{s} \frac{1}{\operatorname{deg}\left(\delta_{s}\right)} \frac{1}{2} \delta_{s, j *}^{2} F_{1}^{2, m}\left(\theta_{s}\right) \cdot[] \circ u_{2, \theta_{s}^{*}} \circ u_{\left(\left(1^{2}: 1^{m}\right)\right)} .
\end{aligned}
$$


Here and elsewhere, we denote by $(n \rightarrow k)$ or, more traditionally $\left(n^{k}\right)$, the distribution $\mu$ with $\mu(n)=k$ and zeros elsewhere; when $n=1$ it will be omitted. Also $\left(\mu_{1}\left|\mu_{2}\right| \ldots \mid \mu_{r}\right)$ denotes the sum as functions $\mu_{1}+\mu_{2}+\ldots+\mu_{r}$; the corresponding diagonal locus is the external or star product $\Gamma_{\mu_{1}} \star \ldots \star \Gamma_{\mu_{r}}$.

Corollary 2.17. We have

$$
\begin{aligned}
\Gamma^{(m)} \cdot \Gamma_{(2)}[] \circ & u_{2, \omega}=\Gamma_{(2 \rightarrow 2)}[] \circ u_{2, \omega}+2 \Gamma_{(3)}[] \circ u_{3, \omega}-\Gamma_{(2)}[] \circ u_{2, \omega^{2}} \\
\Gamma^{(m)} \cdot \Gamma_{(3)}= & \frac{1}{2} \Gamma_{(3 \mid 2)}+3 \Gamma_{(4)}-3 \Gamma_{(3)}[\omega] \\
& +\sum_{s} \frac{3}{\operatorname{deg}\left(\delta_{s}\right)} \sum_{a=0}^{m-3} \delta_{s, 1 *}^{3}\left(F_{1}^{3, m}\left(\theta_{s}\right)+\delta_{s, 2 *}^{3} F_{2}^{3, m}\left(\theta_{s}\right)\right) .
\end{aligned}
$$

Proof. The first formula follows from the fact that the boundary term involves $\theta^{*}$, so drops out after multiplying by $\omega$ because $\theta^{*}(\omega)$ is trivial. The second formula is straight substitution. NB this formula, and similar ones below in cycle form, imply analogous ones in operator from that we will leave to the reader to explicate.

COROllary 2.18. We have for $m=2$ :

$$
\left.\left(\Gamma^{(2)}\right)^{k} \Gamma_{\left(1^{2}\right)}=\frac{1}{2} \Gamma_{(2)}\left[(-\omega)^{k-1}\right]+\sum_{s} \frac{1}{\operatorname{deg}\left(\delta_{s}\right)} \frac{1}{2} \delta_{s, 1 *}^{2}\left(\Gamma^{(2)}\right)^{k-2} \cdot F_{1}^{2,2}\left(\theta_{s}\right)\right), k \geq 3
$$

if $m=2, \operatorname{dim}(B)=1$,

$$
\int_{X_{B}^{[2]}}\left[\Gamma^{(2)}\right]^{3}=\frac{1}{2} \omega^{2}-\frac{1}{2} \sigma, \quad \sigma=\mid\{\text { singular fibres }\} \mid ;
$$

for $m=3$

$$
\begin{gathered}
\left(\Gamma^{(3)}\right)^{3} \Gamma_{\left(1^{3}\right)}=-4 \Gamma_{(3)}[\omega]+\Gamma^{(3)}\left[\omega^{2}\right] \\
+\sum_{s} \frac{1}{\operatorname{deg}\left(\delta_{s}\right)}\left(3\left(\delta_{s, 1 *}^{3} F_{1}^{3,3}\left(\theta_{s}\right)+\delta_{s, 2 *}^{3} F_{2}^{3: 3}\left(\theta_{s}\right)\right)+\frac{1}{2} \delta_{s, 1 *}^{2} \Gamma^{(3)}\left(F_{1}^{2,3}\left(\theta_{s}\right)\right)\right)
\end{gathered}
$$

[We have used the elementary fact that $\omega \cdot \theta_{s}=0$, hence $\omega^{i} \cdot F_{1}^{2: *}\left(\theta_{s}\right)=0, \forall i>0$, because this node scroll maps to $\theta_{s}$, more precisely to $2\left[\theta_{s}\right] \subset X_{B}^{(2)}$.] Note that the last term in the last equation is minus half a node section over $X^{\theta_{s}}$, therefore its support maps birationally to $X_{s}^{\theta}$. Despite the $1 / 2$ factor, the cycles in question are all integral because $\Gamma^{(m)}$ is integral and Cartier (albeit non-effective). In particular, (2.3.14) implies that $\omega^{2}-\sigma$ is even.

To simplify notation we shall henceforth denote $\frac{1}{\operatorname{deg}\left(\delta_{s}\right)} \sum_{s} F_{\bullet}^{\bullet}\left(\theta_{s}\right)$ simply as $F_{\bullet}^{\bullet}$.

EXAMPLE 2.19. This is presented here mainly as a check on some of the coefficients in the formulas above. For $X=\mathbb{P}^{1}, \quad X^{(m)}=\mathbb{P}\left(H^{0}\left(\mathcal{O}_{X}(m)\right)\right)=\mathbb{P}^{m}$, and the degree of $\Gamma_{(n)}^{(m)}$ is $n(m-n+1)$. Indeed this degree may be computed as the degree of the degeneracy locus of a generic map $n \mathcal{O}_{X} \rightarrow P_{X}^{n-1}\left(\mathcal{O}_{X}(m)\right)$ 
where $P_{X}^{k}$ denotes the $k$-th principal parts or jet sheaf. It is not hard to show that $P_{X}^{n-1}\left(\mathcal{O}_{X}(m)\right) \simeq n \mathcal{O}_{X}(m-n+1)$.

For example, $\Gamma_{(2)}^{(3)}$ is a quartic scroll equal to the tangent developable of its cuspidal edge, i.e. the twisted cubic $\Gamma_{(3)}^{(3)}$. The rulings are the lines $L_{p}=\{2 p+q: q \in X\}$, tangent to the $\Gamma_{(3)}^{(3)}$, each of which has class $-\frac{1}{2} \Gamma^{(3)}[\omega]$. Therefore by Corollary 2.16, the self-intersection of $\Gamma^{(3)}$ in $\mathbb{P}^{3}$ (or half the intersection of $\Gamma^{(3)}$ with $\Gamma_{2}^{(3)}$, as a class on $\left.\Gamma_{2}^{(3)}\right)$ is represented by $\Gamma_{(3)}^{(3)}$ plus one ruling $L_{p}$.

If $m=4$ then $\Gamma^{(4)}$ is formally a cubic (half a sextic hypersurface) in $\mathbb{P}^{4}$, whose self-intersection, as given by Corollary 2.16 , is half the Veronese $\Gamma_{(2 \mid 2)}$ plus the (sextic) tangent developable $\Gamma_{(3)}$, plus one osculating plane to the twisted quartic $\Gamma_{(4)}$, representing $-\Gamma^{(4)}[\omega]$.

Next we extend Proposition 2.15 to the polyblock case, in other words work out the unordered analogue of Corollary 2.13. Consider a distribution $\mu$ of weight $m$ and associated polyblock diagonal loci and operators $D_{\mu}, \Gamma_{\mu}, \Gamma_{\mu}[]$ where, e.g.

$$
\Gamma_{\mu}[]: \operatorname{TS}_{\mu}(R) \rightarrow A_{\bullet}\left(X_{B}^{[m]}\right)
$$

$A_{\bullet}$ could be replaced by for any suitable homology theory $H_{\bullet}$ such as singular (over $\mathbb{Q})$. The group becomes a ring whenever $X$ is smooth, hence so is $X_{B}^{[m]}$. Now the node scroll $F_{j}^{n}(\theta)$ (see the next section for more detail) is a $\mathbb{P}^{1}$-bundle over $\left(X_{T}^{\theta}\right)^{[m-n]}$, whence operators, for any distribution $\nu$ of weight $m-n$ :

$$
\begin{array}{r}
F_{j, \nu}^{n}(\theta)[]: \operatorname{TS}_{\nu}(R) \rightarrow A_{\bullet}\left(X_{B}^{[m]}\right) \\
\alpha . \mapsto\left[F_{j}^{n}(\theta)\right] \cap p_{[m-n]}^{*} \circ \Gamma_{\nu, X_{T}^{\theta}} \circ \phi^{*}(\alpha .)
\end{array}
$$

where $\phi: X_{T}^{\theta} \rightarrow X$ is the natural map. Clearly, given a distribution $\mu$ of weight $m$, the $\nu$-s corresponding to it via the unordered analogue of Corollary 2.13 will have the form $\nu=\mu-1_{n}$ with $\mu(n) \geq 1$.

A convenient way to represent the classes $\Gamma_{\mu}[\alpha]$ and $F_{j, \nu}^{n}(\theta)[\alpha]$, adopted in the macnodal program (see §4.3) is as matrices where the first row represent the partition $\mu$ and each column has header $n$ and beneath it a vector representation of the corresponding class.

Now the following result, which is the proper Hilbert scheme analogue of Lemma 1.2 , follows directly from Corollary 2.13 by adjusting for the degrees of the various symmetrization maps. [NB The factor of $\frac{1}{\mu(n)}$ in the boundary term on the RHS is due to the fact that In the boundary term, the relevant ordered node scrolls map to their unordered versions with degree $\prod_{p \neq n} \mu(p) !(\mu(n)-1)$ !, whereas $\Gamma_{(I .)}$ maps to its unordered version $\Gamma_{\mu}$ with degree $\prod_{p \neq n} \mu(p) !(\mu(n))$ !. This introduces a factor of $1 / \mu(n)$, which gets canceled as there are $\mu(n)$ terms of this type.]

Proposition 2.20. For a distribution $\mu$ of weight $m$, we have an equality of operators $\operatorname{TS}_{\mu}(R) \rightarrow A_{\bullet}\left(X_{B}^{[m]}\right)$ :

$$
\Gamma^{(m)} . \Gamma_{\mu}[]=\Gamma_{\mu} \circ\left(\operatorname{Dsc}^{(m)}-U_{\omega}\right)+\sum_{s} \sum_{\mu(n)>0} \sum_{j=1}^{n-1} \frac{1}{2} j(n-j) n F_{j, \mu-1_{n}}^{n, m}\left(\theta_{s}\right)[] \circ u_{n, \theta_{s}^{*}, \mu}
$$


EXAMPLE 2.21

$$
\begin{aligned}
\Gamma^{(m)} . \Gamma_{(2 \rightarrow 2)}[]= & \frac{3}{2} \Gamma_{(2 \rightarrow 3)}[] \circ u_{1,1}+2 \Gamma_{(4)}[] \circ u_{2,2}+2 \Gamma_{(3 \mid 2)}[] \circ u_{2,1} \\
& -\Gamma_{(2 \rightarrow 2)}[] \circ u_{2, \omega}+\sum_{s} F_{1}^{2, m}[] \circ u_{2, \theta_{s}^{*} .} \square
\end{aligned}
$$

EXAMPLE 2.22

$$
\begin{aligned}
\left(\Gamma^{(m)}\right)^{3} \Gamma_{\left(1^{m}\right)}[] & =\frac{3}{4} \Gamma_{(2 \rightarrow 3)}+4 \Gamma_{(4)}+\frac{3}{2} \Gamma_{(3 \mid 2)}-\Gamma_{(2 \rightarrow 2)} \circ u_{2, \omega}-4 \Gamma_{(3)} \circ u_{3, \omega} \\
+ & \frac{1}{2} \Gamma_{(2)} \circ u_{2, \omega^{2}}+\frac{1}{4} F_{1,(2)}^{2, m}+3\left(F_{1}^{3, m}+F_{2}^{3, m}\right)+\frac{1}{2} \Gamma^{(m)} \cdot F_{1}^{2, m} . \square
\end{aligned}
$$

Now assembling the various $\Gamma_{\mu}$ together, we obtain our definitive result on multiplying generalized (twisted) diagonal cycles by the discriminant polarization:

THEOREM 2.23. We have an equality of operators $\operatorname{TS}(R) \rightarrow A_{\bullet}\left(X_{B}^{[m]}\right)$ :

$$
\begin{aligned}
\Gamma^{(m)} \cdot \Gamma \bullet m[]= & \Gamma \bullet m \circ\left(\operatorname{Dsc}^{(m)}-U_{\omega}\right) \\
& +\sum_{\mu} \sum_{s} \sum_{\mu(n)>0} \sum_{j=1}^{n-1} \frac{1}{2} j(n-j) n F_{j, \mu-1_{n}}^{n, m}\left(\theta_{s}\right)[] \circ u_{n, \theta_{s}^{*}, \mu}
\end{aligned}
$$

Recall from Lemma 1.2 that $\operatorname{Dsc}^{(m)}-U_{\omega}$ represents the action of the discriminant on the the various diagonals put together. Therefore the Theorem can be viewed as a 'commutation relation' for this action: the failure of commutativity is measured by the node scrolls. The nontrivial part is determining the multiplicities with which they occur.

Because we want the Tautological Module $T^{m}$ (yet to be defined) to include the $\Gamma_{\mu}[]$, it must also include multiples of these by powers of the polarization $\Gamma^{(m)}$. Therefore by the above, $T^{m}$ must also include the (twisted) node scrolls $F=F_{j, \nu}^{n}(\theta)[]$ and their multiples by powers of $\Gamma^{(m)}$. Fortunately, it turns out that including the twisted scrolls $F$ and their first-degree multiples $\Gamma^{(m)} F$ already leads to closure; moreover, products of all these by arbitrary powers of $\Gamma^{(m)}$ can be computed. In essence, this is accomplished by the Node Scroll Theorem of [19]. The details are taken up in the next section.

2.4. Polarized node scrolls. Before taking up the node scrolls, we mention an elementary analogue. Suppose the family $X / B$ admits a relative Carter divisor $\rho$, which is flat over $B$ of degree $k$. Then there is an induced 'incrementation' map

$$
\rho_{+}: X_{B}^{[m]} \rightarrow X_{B}^{[m+k]}
$$

which send an ideal $z$ to $z \cdot \mathcal{O}(-\rho)$. In particular, if $X / B$ admits a section $\theta$ - necessarily supported in smooth points- we get maps $(k \theta)_{+}$. The node scrolls are analogues of this construction where $k \theta$ is replaced by a subscheme supported on a relative node of $X / B$.

We recall from [19] that the node scroll $F_{j}^{n, m}(\theta)$ (fixing $m$ ) are correspondences

$$
p_{[m-n]} \stackrel{F_{j}^{n, m}(\theta) \stackrel{p_{[m]}}{\rightarrow} X_{B}^{[m]}}{\stackrel{\downarrow}{\downarrow}} \underset{ }{\left(X_{T}^{\theta}\right)^{[m-n]}}
$$


where $p_{[m]}$ is generically finite onto a component the locus of schemes having length at least $n$ at $\theta$, while $p_{[m-n]}$ is a $\mathbb{P}^{1}$-bundle projection. Note that $F_{j}^{n, m}(\theta)$ defines an operator

$$
\begin{array}{r}
A_{\bullet}\left(\left(X^{\theta}\right)_{T}^{[m-n]}\right) \rightarrow A_{\bullet}\left(X_{B}^{[m]}\right), \\
\beta \mapsto p_{[m] *} p_{[m-n]}^{*}(\beta) .
\end{array}
$$

We will however view $F_{j}^{n, m}(\theta)$ as acting just on the tautological module $T^{m-n}\left(X_{T}^{\theta}\right)$, which we may assume defined by induction on Hilb degree (more on this shortly), and as such its image will be in $T^{m}(X / B)$. We will call $F_{j}^{n, m}(\theta)[\beta]$ for $\beta \in T^{m-n}\left(X_{T}^{\theta}\right)$ a twisted node scroll class (of Hilb degree $m$ on $X / B$ ). The polarized structure of the node scroll $F_{j}^{n, m}(\theta)$, refers to its description as projectivization of a particular rank-2 vector bundle (in fact, a direct sum of two explicit line bundles) on the degree$(m-n)$ Hilbert scheme $\left(X_{T}^{\theta}\right)^{[m-n]}$, with the property that the associated $\mathcal{O}(1)$ relative polarization coincides with $-p_{[m]}^{*}\left(\Gamma^{(m)}\right)$. This was worked out in [19] and can be described as follows.

Fix a boundary family $X_{T}^{\theta}$ and let $\theta_{x}, \theta_{y}$ be the sections of $X_{T}^{\theta}$ mapping to the node $\theta$, and let

$$
\psi_{x}=\theta_{x}^{*}\left(\omega_{X^{\theta} / T}\right),
$$

considered as a line bundle on $T$ (and by pullback, on any space mapping to $T$ ). As in $\S 1.6$, let $[k]_{*} L$ be the $k$-th norm associated to a line bundle $L$ on $X$ (which is a divisor class on $X_{B}^{[k]}$ ). Then set

$$
D_{j}^{n, m}(\theta)=\left(\begin{array}{c}
n-j+1 \\
2
\end{array}\right) \psi_{x}+\left(\begin{array}{l}
j \\
2
\end{array}\right) \psi_{y}-(n-j+1)[m-n]_{*} \theta_{x}-j[m-n]_{*} \theta_{y}
$$

(confusing divisors and line bundles on $\left(X^{\theta}\right)_{T}^{[m-n]}$ ). The Node Scroll theorem of [19] yields an isomorphism

$$
F_{j}^{n, m}(\theta) \simeq \mathbb{P}\left(\mathcal{O}\left(D_{j}^{n, m}(\theta)\right) \oplus \mathcal{O}\left(D_{j+1}^{n, m}(\theta)\right)\right)
$$

under which

$$
-p_{[m]}^{*}\left(\Gamma^{(m)}\right)+p_{[m-n]}^{*}\left(\Gamma^{(m-n)}\right) \leftrightarrow \mathcal{O}(1) .
$$

To make use of this, set

$$
e_{j}^{n, m}(\theta)=\left[D_{j}^{n, m}(\theta)\right]-\Gamma^{(m-n)} \in A^{1}\left(\left(X^{\theta}\right)_{T}^{[m-n]}\right) .
$$

Of course, $\Gamma^{(m-n)}=0$ if $m-n \leq 1$. Thus, the $\Gamma^{(m-n)}$ term begins to appear only for $m \geq 4$. We will identify this class with its pullback on $F_{j}^{n, m}(\theta)$. Then the $e_{j}^{n, m}(\theta)$, and polynomials in them, also define operators on classes on $X_{T}^{\theta}$. Also, set formally

$$
s_{k}(a, b)=a^{k}+a^{k-1} b+\ldots+b^{k} \quad\left({ }^{\prime}=\frac{a^{k+1}-b^{k+1}}{a-b}\right) .
$$

Thus,

$$
s_{k}\left(e_{j}^{n, m}, e_{j+1}^{n, m}\right)=\frac{\left(e_{j+1}^{n, m}\right)^{k+1}-\left(e_{j}^{n, m}\right)^{k+1}}{-(n-j) \psi_{x}+j \psi_{y}+\theta_{x}-\theta_{y}} .
$$


Then the Node Scroll Theorem plus the usual relation of Chern and Segre classes yield immediately

TheOREM 2.24. For any twisted node scroll class $F_{j}^{n, m}(\theta)[\beta]$, we have

$$
\begin{aligned}
\left(-\Gamma^{(m)} \cdot\right)^{\ell} \cdot F_{j}^{n, m}(\theta)[\beta]= & \left(-\Gamma^{(m)}\right) F_{j}^{n, m}(\theta)\left[s_{\ell-1}\left(e_{j}^{n, m}, e_{j+1}^{n, m}\right) \beta\right] \\
& -F_{j}^{n, m}(\theta)\left[e_{j}^{n, m} e_{j+1}^{n, m} s_{\ell-2}\left(e_{j}^{n, m}, e_{j+1}^{n, m}\right) \beta\right]
\end{aligned}
$$

[The first term is just the definition of Segre class; to get the second term, work inductively and use the $(\ell-1)$ case and the Grotendieck formula (i.e. the $\ell=2$ case).]

The class $-\Gamma^{(m)} \cdot F_{j}^{n}(\theta)$, called a node section, projects with degree 1 to $\left(X^{\theta}\right)_{T}^{[m-n]}$. Evaluating the rest of the RHS of 2.4.8 involves, essentially, the tautological module in lower degree and, in case $X / B$ is a family of stable curves, lower genus as well, albeit for a family of pointed curves $X_{T}^{\theta}$, with distinguished sections $\theta_{x}, \theta_{y}$. To evaluate the terms involving these, we may note the following elementary formulas, in which $\theta$ denotes any section and $\psi=\pi_{*}\left(\left.\omega\right|_{\theta}\right)$ :

$$
\begin{gathered}
(\theta)^{r}=(-\psi)^{r-1} \theta, r \geq 1 ; \theta_{x} \theta_{y}=0 ; \\
\left([k]_{*} \theta\right)^{t}=\sum_{s=1}^{\min (k, t)}\left(s^{t}-(s-1)^{t}\right)(-\psi)^{t-s}[k]_{*}^{s}(\theta)
\end{gathered}
$$

where we recall, cf. (1.6.20), that $[k]_{*}^{s}(\theta)$ denotes the symmetrization of $\theta^{\times s}$ and its pullback on the Hilbert scheme.

Proof of (2.4.10). Clearly,

$$
\left([k]_{*} \theta\right)^{t}=\sum_{s} \sum_{\substack{r_{1}+\ldots+r_{s}=t \\
r_{i} \geq 1 \forall i}}\left(\begin{array}{c}
t \\
r_{1}, \ldots, r_{s}
\end{array}\right)(-\psi)^{t-s}[k]_{*}^{s}(\theta) .
$$

To evaluate the numerical coefficient, say $a_{s}$, note that

$$
a_{1}+\ldots+a_{s}=\sum\left(\begin{array}{c}
t \\
r_{1}, \ldots, r_{s}
\end{array}\right)=s^{t}
$$

hence $a_{s}=s^{t}-(s-1)^{t}$.

The pullback of (2.4.10) on a polyblock diagonal $\Gamma_{\nu}$ is given by the $D_{\nu}^{\dagger}$ operator defined in $\S 1.3$, viz.

$$
\Gamma_{\nu} \cdot\left([k]_{*} \theta\right)^{t}=\sum_{s=1}^{\min (k, t)}\left(s^{t}-(s-1)^{t}\right)(-\psi)^{t-s} \Gamma_{\nu}\left[D_{\nu}^{\dagger}\left([k]_{*}^{s}(\theta)\right)\right]
$$

Similarly, on the operator level,

$$
\Gamma_{\nu} \cdot\left([k]_{*} \theta\right)^{t}[\beta]=\sum_{s=1}^{\min (k, t)}\left(s^{t}-(s-1)^{t}\right)(-\psi)^{t-s} \Gamma_{\nu}\left[D_{\nu}^{\dagger}\left([k]_{*}^{s}(\theta) \cdot \beta\right)\right]
$$


for $\beta \in \operatorname{TS}_{\nu}\left(H \cdot\left(X_{T}^{\theta}\right)\right.$ ) (where the.$\beta$ means formal symmetric multiplication). In particular, using the inductive case of Theorem 2.1, it follows that the bracketed expressions appearing in Theorem 2.24 are all tautological classes, therefore

Corollary 2.25. Notations as above, $\left(-\Gamma^{(m)} .\right)^{\ell} \cdot F_{j}^{n}(\theta)[\beta]$ is a twisted node scroll class.

REMARK 2.26. Given the canonical, mutually disjoint sections

$$
Q_{i}^{n, m}=\mathbb{P}\left(\mathcal{O}\left(e_{i}^{n, m}\right)\right), Q_{i+1}^{n, m} \subset F_{i}^{n, m}
$$

we can write the node section in the form

$$
-\Gamma^{(m)} F_{i}^{n, m}=Q_{i}^{n, m}+\pi^{*}\left(e_{i+1}^{n, m}\right) .
$$

Consequently,

$$
\begin{aligned}
-\Gamma^{(m)} F_{i}^{n, m}= & Q_{1}^{n, m}+\sum_{1 \leq j<i} F_{j}^{n, m}\left[[m-n]_{*}\left(\theta_{x}-\theta_{y}\right)-(n-j) \psi_{x}+j \psi_{y}\right] \\
& +F_{i}^{n, m}\left[e_{i}^{n, m}\right] .
\end{aligned}
$$

Consequently, rather than work with the $n-1$ node sections $-\Gamma^{(m)} F_{i}^{n, m}, i=1, \ldots, n-$ 1 , one could instead work with a single canonical section like $Q_{1}^{n, m}$, together with various twisted node scrolls.

EXAMPLE 2.27. Note that when $\operatorname{dim}(B)=1$, so $T$ is a point, we have $\theta_{x} \sim$ $\theta_{y} \sim \theta_{0}$, a point on $X^{\theta}$. Therefore $D_{j}^{n, m}(\theta)$ is independent of $j$ up to numerical equivalence, hence $F_{j}^{n, m}(\theta)$ is also, for all $j$, deformation-equivalent, hence has the same intersection theory, as the trivial $\mathbb{P}^{1}$-bundle $\left(X^{\theta}\right)^{(m-n)} \times \mathbb{P}^{1}$, so that $\Gamma^{(m)} \sim$ $\Gamma_{\left(X^{\theta}\right)^{(m-n)}}+(n+1)[m-n]_{*}\left(\theta_{0}\right)-h_{\mathbb{P}^{1}}$. Therefore

$$
\begin{aligned}
\left(\Gamma^{(m)}\right)^{k} . F_{j}^{n, m} \sim & \left(\Gamma_{\left(X^{\theta}\right)^{(m-n)}}+(n+1)[m-n]_{*}\left(\theta_{0}\right)\right)^{k} \\
& -k\left(\Gamma_{\left(X^{\theta}\right)^{(m-n)}}+(n+1)[m-n]_{*}\left(\theta_{0}\right)\right)^{k-1} \cdot h_{\mathbb{P}^{1}} .
\end{aligned}
$$

See Example 2.34 for an evaluation of these cycles.

EXAMPLE 2.28. We have

$$
F_{1}^{(2,3)}(\theta)=\mathbb{P}_{X^{\theta}}\left(\mathcal{O}\left(-2 \theta_{x}-\theta_{y}\right) \oplus \mathcal{O}\left(-\theta_{x}-2 \theta_{y}\right)\right)
$$

Consequently, if the boundary is finite,

$$
\left(-\Gamma^{(3)}\right)^{2} \cdot F_{1}^{(2,3)}=-6 .
$$

Note that in the 'extreme' case $m=n$, the $e_{j}^{n}(\theta)$ and the node scroll $F_{j}^{n}(\theta)$ live on the base itself $T$ of the boundary datum and we have

$$
e_{j}^{n}(\theta)=\left(\begin{array}{c}
m-j+1 \\
2
\end{array}\right) \psi_{x}+\left(\begin{array}{l}
j \\
2
\end{array}\right) \psi_{y}:=\psi_{j}^{m} .
$$


EXAMPLE 2.29. For $m=n=2, F=F_{1}^{2}(\theta)=\mathbb{P}\left(\psi_{x} \oplus \psi_{y}\right)$, we have

$$
\begin{aligned}
\left.\left(-\Gamma^{(2)}\right)^{k}\right|_{F}= & \left(\psi_{x}^{k-1}+\psi_{x}^{k-2} \psi_{y}+\ldots+\psi_{y}^{k-1}\right)\left(-\Gamma^{(2)}\right) \\
& -\psi_{x} \psi_{y}\left(\psi_{x}^{k-2}+\psi_{x}^{k-3} \psi_{y}+\ldots+\psi_{y}^{k-2}\right) .
\end{aligned}
$$

In particular, for $k=\operatorname{dim}(B)=\operatorname{dim}(F)=1+\operatorname{dim}(T)$, which is when the class becomes 0-dimensional, we have for its degree

$$
\left(-\Gamma^{(2)}\right)^{k} \cdot F=\int_{T}\left(\psi_{x}^{k-1}+\psi_{x}^{k-2} \psi_{y}+\ldots+\psi_{y}^{k-1}\right) .
$$

Note that if $B=\overline{\mathcal{M}}_{g}$ and $T=\overline{\mathcal{M}}_{i, 1} \times \overline{\mathcal{M}}_{g-i, 1}, 1 \leq i \leq g / 2$ (the usual $i$-th boundary component), only one summand contributes to the latter integral, which reduces to

$$
\int_{\mathcal{M}_{i}} \psi_{x}^{3 i-2} \int_{\overline{\mathcal{M}}_{g-i}} \psi_{y}^{3(g-i)-2} .
$$

Note that (2.4.17) and (2.3.13) together imply

Corollary 2.30. (i) The powers of the polarization on $X_{B}^{[2]}$ are

$$
\begin{aligned}
\left(-\Gamma^{(2)}\right)^{k}= & -\Gamma\left[\omega^{k-1}\right]+\frac{1}{2} \sum_{s} \delta_{s *}\left(\left(\psi_{x}^{k-3}+\psi_{x}^{k-4} \psi_{y}+\ldots+\psi_{y}^{k-3}\right)\left(-\Gamma^{(2)}\right)\right. \\
& \left.-\left.\psi_{x} \psi_{y}\left(\psi_{x}^{k-4}+\psi_{x}^{k-5} \psi_{y}+\ldots+\psi_{y}^{k-4}\right)\right|_{F_{1}^{2,2}\left(\theta_{s}\right)}\right)
\end{aligned}
$$

(ii) The image of the latter class on the symmetric product $X_{B}^{(2)}$ equals

$$
-\Gamma\left[\omega^{k-1}\right]+\frac{1}{2} \sum_{s} \delta_{s *}\left(\left(\psi_{x}^{k-3}+\psi_{x}^{k-4} \psi_{y}+\ldots+\psi_{y}^{k-3}\right)\right.
$$

(iii) The image of the latter class on $B$ equals $-\kappa_{k-2}+\frac{1}{2} \sum_{s} \delta_{s *}\left(\left(\psi_{x}^{k-3}+\psi_{x}^{k-4} \psi_{y}+\right.\right.$ $\left.\ldots+\psi_{y}^{k-3}\right)$.

Proof. (i) has been proved above; (ii) follows because in the last summation in (2.4.19), the terms without $\Gamma^{(2)}$, i.e. the twisted node scroll, collapses under the cycle map to $X_{B}^{(2)}$; (iii) follows similarly.

REMARK 2.31. It is interesting to compare the above boundary term with the boundary term in Mumford's formula [14] for the Chern character of the Hodge bundle; our $\psi_{x}, \psi_{y}$ are his $K_{1}, K_{2}$, and $\psi_{x} \oplus \psi_{y}$ is the conormal bundle to $\theta$ in $X$; so our term is essentially the Segre class of $\theta$ in $X$, while Mumford's term is a Todd class of the same.

EXAMPLE 2.32. $m=3, n=2, \operatorname{dim}(B)=1$ :

$$
\left(-\Gamma^{(3)}\right)^{2} \cdot F_{1}^{(2: 3)}(\theta)=-6
$$

(see Example 2.28). Consequently, in view of Corollary 2.18, we conclude that if $X / B$ is a 'good pencil': i.e. smooth total space and base, all singular fibres 1-nodal, then, where $\sigma$ denotes the number of singular fibres, we have

$$
\int_{X_{B}^{[3]}}\left(\Gamma^{(3)}\right)^{4}=13 \omega^{2}-9 \sigma
$$


(recall that each $F_{i}^{(3,3)}, i=1,2$ is a line with respect to the discriminant polarization $\left.-\Gamma^{(3)}\right)$.

EXAMPLE 2.33. In general, each $F^{m, m}(\theta)$ is a cycle of $\mathbb{P}^{1}$-bundles over the appropriate boundary component, whose total degree (with respect to $-\Gamma^{(m)}$ ) is given by $\nu_{m}=\frac{m^{2}\left(m^{2}-1\right)}{12}$ (cf. Corollary 2.7$)$. In particular, for a good pencil, we get

$$
\left(-\Gamma^{(m)}\right) F^{m, m}=\sigma \frac{m^{2}\left(m^{2}-1\right)}{12} .
$$

In the ensuing examples, we will explore a non-recursive approach to some questions in tautological enumerative geometry based on graph enumeration. Some closely related arguments were discovered independently and earlier by Cotteril [3].

EXAMPLE 2.34. Let $X / B$ be a single smooth curve of genus $g$ over a point, and set

$$
f_{m, g}=\int_{X_{B}^{(m)}}\left(\Gamma^{(m)}\right)^{m}=\frac{1}{m !} \int_{X_{B}^{m}}\left(\Gamma^{(m)}\right)^{m} .
$$

Because for genus 0 the discriminant is a hypersurface of degree $m-1$ in $\left(\mathbb{P}^{1}\right)^{(m)}=\mathbb{P}^{m}$, we have

$$
f_{m, 0}=(m-1)^{m}
$$

Consider the generating function

$$
f_{g}(z)=1+\sum_{m=2}^{\infty} f_{m, g} z^{m}
$$

In particular,

$$
f_{0}(z)=1+\sum_{m=2}^{\infty} \frac{(m-1)^{m}}{m !} z^{m}
$$

Let $W_{b}(z)$ be as in $\S 1.1$. Then by viewing $f_{m, g}$ as $\frac{1}{m !} \int_{X_{B}^{m}}\left(\sum_{i<j} D_{i, j}\right)^{m}$, we will show that

$$
f_{g}(z)=\exp \left(2(1-g) W_{1}(z)\right)
$$

In particular,

$$
f_{g}(z)=\left(f_{0}(z)\right)^{1-g}=\left(1+\sum_{m=2}^{\infty} \frac{(m-1)^{m}}{m !} z^{m}\right)^{1-g}
$$

Also,

$$
W_{1}(z)=\frac{1}{2} \log \left(f_{0}(z)\right)
$$


To prove (2.4.24), expand $\left(\sum D_{i, j}\right)^{m}$ multinomially, and attach to each monomial $M$ an edge-weighted graph with vertex-set the set of indices occurring in $M$ and with an edge for each $D_{i, j}$ occurring in $M$ (i.e. the multiplicity $m_{i, j}$ of the edge $(i, j)$ equals the exponent of $D_{i, j}$ in $\left.M\right)$. This graph is assigned a weight of $\frac{1}{\prod m_{i, j} !}$ due to the multinomial coefficient $\frac{m !}{\prod m_{i, j} !}$. A 'connected' monomial, i.e. one with connected graph, with $n$ vertices and $n$ edges, will contribute $2(1-g) w_{n, n}$ to the degree, and a general monomial will contribute the product of the contributions of its connected components. Then standard generating function techniques yield the above formula.

More generally, consider the divisor class $\theta=\theta_{m}=\left[p+X^{(m-1)}\right]=[m]_{*}(p t) \subset$ $X^{(m)}$ and let

$$
h_{m}(u, g)=\int_{X^{(m)}}\left(\Gamma^{(m)}+u \theta\right)^{m} .
$$

Now because $\Gamma^{(m)} \cdot X^{(m-1)}=\Gamma^{(m-1)}+\theta_{m-1}$, we have

$$
h_{m}(u, g)=f_{m, g}+u f_{m-1, g}(u+1) .
$$

Therefore

$$
h_{m}(u, g)=\sum_{i=0}^{m-1} f_{m-i, g} u \ldots(u+i-1) .
$$

In particular, when $u$ is an integer we get

$$
h_{m}(u, g)=\int_{X^{(m)}}\left(\Gamma^{(m)}+u \theta_{m}\right)^{m}=\sum_{i=0}^{\infty} i !\left(\begin{array}{c}
u+i-1 \\
i
\end{array}\right) f_{m-i, g} .
$$

Such formulas could persumably also be obtained by Macdonald's intersection theory (see Remark 1.4); however his methods don't seem adaptable to the singular case .

Referring back to Example 2.27, it follows that in the good pencil case, the degree of the node scroll $F_{j}^{n, m}(\theta)$ is computed by

$$
\int_{F_{j}^{n, m}(\theta)}\left(-\Gamma^{(m)}\right)^{m-n+1}=(-1)^{m-n}(m-n+1) h_{m-n}(n+1, g-1) .
$$

See Example 2.39 for a generalization.

EXAMPLE 2.35. In the good pencil case, Theorem 2.23 shows that the degree of the discriminant polarization has the form

$$
\int_{X_{B}^{[m]}}\left(\Gamma^{(m)}\right)^{m+1}=f_{m, g}^{1} \omega^{2}+b_{m, g} \sigma
$$

with coefficients universal rational numbers. The coefficient $f_{m, g}^{1}$, which comes from the first, 'classical' summand in (2.3.20), can be determined via the expression (1.4.13) (or by working with any particular smooth pencil with $\omega^{2} \neq 0$ ). Writing formally

$$
f_{m, g}^{1}=\frac{1}{m !} \int_{X_{B}^{m}}\left(\sum_{i<j} D_{i, j}\right)^{m+1}
$$


with the $D_{i, j}$ as in (1.4.13) (where in operator terms the integral signifies 'apply on $\left.1^{\otimes m}\right)$, we can again relate these to graph numbers. Consider the generating function

$$
f_{g}^{1}(z)=1+\sum_{m=2}^{\infty} f_{m, g}^{1} z^{m} .
$$

Then as in Example 2.34, we have

$$
W_{2}(z) \exp \left(2(1-g) W_{1}(z)\right)=f_{g}^{1}(z)
$$

i.e.

$$
f_{g}^{1}(z)=W_{2}(z)\left(f_{0}(z)\right)^{1-g}
$$

(again $W_{2}(z)$ is as in $\left.\S 1.1\right)$. This is because the monomials computing $f_{m, g}^{1}$ correspond to graphs with one connected component of Betti number 2 and all others of Betti number 1.

EXAMPLE 2.36. More generally we can compute, for arbitrary base dimension, the polyblock diagonal $\Lambda_{m, k}$ (modulo node classes ) portion of $\frac{1}{k !}\left(\Gamma^{(m)}\right)^{k}$. To state the result, we need the notion of pair-partition or ppartition. By definition, a ppartition $\rho$ is an unordered collection of ordered pairs $(n, b), n>0, b \geq 0$. Formally, a ppartition is a function $\rho: \mathbb{N} \times \mathbb{Z}_{\geq 0} \rightarrow Z_{\geq 0}$ where $\rho(n, b)$ counts the frequency of a block pair of sizes $n, n+b$. The underlying partition of $\rho$ is by definition the partitiion $\mu=\rho_{\dagger}$ defined by

$$
\mu(n)=\sum_{b} \rho(n, b)
$$

Heuristically, we think of a ppartition $\rho$ as consisting of a partition $\rho_{\dagger}$, together with a choice of nonnegative 'exponent' $b$ for each block, so that $\rho(n, b)$ counts the size- $n$ block with exponent $b$. The 'upper partition' $\mu^{\prime}=\rho^{\dagger}$ of $\rho$ is defined as

$$
\mu^{\prime}(b)=\sum_{n} \rho(n, b) .
$$

we define the degree of $\rho$ as

$$
\operatorname{deg}(\rho)=\sum(n+b-1) \rho(n, b)
$$

An ordinary partition is naturally viewed as a pppartition with all exponents $b=0$. Our motivation for this definition is as follows. Given a family $X / B$, we can associate to $\rho$ a polyblock diagonal class on $X_{B}^{[m]}, m=\left|\rho_{\dagger}\right|$ :

$$
\Gamma_{\rho}[(-\omega)]=\prod_{n, b} \Gamma_{(n)}\left[(-\omega)^{b}\right]^{\star \rho(n, b)}
$$

where $\star$ is star multiplication, where $\Gamma_{\mu}[\alpha] \star \Gamma_{\nu}[\beta]=\Gamma_{\mu+\nu}[\alpha \beta]$ (see $\S 1.3$ ). Note that a connected monomial in the $D_{i, j}$ yields $\Gamma_{(n)}\left[(-\omega)^{b}\right]$ where $n+b$ is the degree of the monomial (aka number of edges) and $n$ is the number of distinct indices (aka number of vertices). Thus a possibly disconnected monomial yields $\Gamma_{\rho}[(-\omega)]$ for some ppartition $\rho$. 
Let $s=\operatorname{dim}(B)$. Set formally, and analogously as in $\S 1.1$,

$$
\begin{gathered}
\tilde{W}_{b}=\sum_{n} \frac{w_{n, n-1+b}}{n !} \Gamma_{(n)}\left[(-\omega)^{b}\right] \\
\Lambda=\sum_{m, k} \Lambda_{m, k}=\sum_{\rho} L(\rho) \Gamma_{\rho}[(-\omega)]:=\sum_{m, k} \frac{1}{k !}\left(\Gamma^{(m)}\right)^{k} \Gamma_{\left(1^{m}\right)}=\sum_{m, k} \frac{1}{m ! k !}\left(\sum_{1 \leq i<j \leq m} D_{i, j}\right)^{k}
\end{gathered}
$$

(equality modulo node classes). We will also use the notation $\Lambda(X / B)$ etc. when the family needs specification. Note that $(-\omega)^{b}=\tilde{W}_{b}=0$ for $b>s+1$.Then

$$
\Lambda=\exp _{\star}\left(\sum_{b=0}^{s+1} \tilde{W}_{b}\right)
$$

This formula results from the fact that a connected monomial of $n$ vertices and $n-1+b$ edges yields $\Gamma_{(n)}\left[(-\omega)^{b}\right]$.

This approach to deriving explicit formulas for powers of $\Gamma$, and hence also for Chern polynomials in tautological bundles, can be carried substantially further. We will return to this elsewhere (see [17]).

EXAMPLE 2.37. We extend the above example to compute the action of $\left(\Gamma^{(m)}\right)^{k}$ on a twisted polyblock diagonal $\Gamma_{(n .)}[a$.$] , for any partition (n)=.\left(n_{1} \geq \ldots \geq n_{r}\right)$ of weight $m$ and corresponding collection of classes $a_{i} \in R \subset H \cdot(X)$ (the $a_{i}$ can also be taken to be indeterminates). Thus we will compute

$$
\left(\Gamma^{(m)}\right)^{k} . \Gamma_{(n .)}[a .]=: \Lambda_{m, k,(n ., a .)}+\sum F_{j}^{n, m}(\theta)\left[B_{m, k,(n ., a .), n, j}^{\theta}\right]+\sum Q_{j}^{n, m}(\theta)\left[C_{m, k,(n ., a .), n, j}^{\theta}\right] .
$$

Set $p_{i}=n_{i} a_{i}$. The computation is based on the weighted counts $w_{n, p ., m}$ as in (1.1.4). For an index-set $S \subset[r]$, set

$$
n_{S}=\sum_{i \in S} n_{i}, z^{S}=\prod_{i \in S} z_{i}
$$

where $z_{i}$ are indeterminates with $z_{i}^{2}=0$.

$$
\tilde{W}_{(n ., a .), k}=\sum_{S} \frac{w_{|S|,(p .), k}}{|S| !} \Gamma_{\left(n_{S}\right)}\left[(-\omega)^{k+1-|S|}\right] z^{S}
$$

Then we have, with a similar proof as above,

$$
\exp \left(\Gamma^{(m)}\right) \Gamma_{(n .)}[a .]=\sum_{k} \frac{1}{k !} \Lambda_{m, k,(n . c .)} z_{1} \ldots z_{r}=\exp _{\star}\left(\sum_{k)} \tilde{W}_{(n ., a .), k}\right)
$$

ExAmple 2.38. Consider again a single smooth curve $X / B$ of genus $g$. Extending Example 2.34, we now determine the degree

$$
f_{m, \mu, g}=\int_{\Gamma_{\mu}}\left(\Gamma^{(m)}\right)^{m-\ell}
$$


Here $\mu$ is a partition of weight $m$ and length $\ell=\sum \mu(n)$. Indeed in the case $g=0$ it is easy to see that $\Gamma_{\mu}$, which is a subset of $\left(\mathbb{P}^{1}\right)^{(m)}=\mathbb{P}^{m}$, has normalization $\prod_{n} \mathbb{P}^{\mu(n)}$ and the pullback of $\mathcal{O}_{\mathbb{P}^{m}}(1)$ is $\otimes \mathcal{O}_{\mathbb{P}^{\mu(n)}}(n)$. It follows that

$$
f_{m, \mu, 0}=(m-1)^{\ell} \ell ! \prod \frac{n^{\mu(n)}}{\mu(n) !}
$$

Then again if we form the generating function

$$
f_{\mu, g}(z)=1+\sum_{m=2}^{\infty} \frac{f_{m, \mu, g}}{m !} z^{m}
$$

then we have

$$
f_{\mu, g}(z)=\left(f_{\mu, 0}(z)\right)^{1-g}=\left(1+\sum_{m=2}^{\infty} \frac{(m-1)^{\ell} \ell !}{m !} \prod_{n} \frac{n^{\mu(n)}}{\mu(n) !}\right)^{1-g}
$$

As in Example 2.34, we now extend this to compute

$$
h_{\mu, g}(u):=\int_{\Gamma_{\mu}}\left(\Gamma^{(m)}+u \theta\right)^{m-\ell}, m:=|\mu| .
$$

Indeed note that

$$
\theta \cdot \Gamma_{\mu}=\sum_{n} n \Gamma_{\mu-1_{n}}, \Gamma_{\mu-1_{n}} \subset X^{(m-n)}
$$

where $\mu-1_{n}$ is the partition obtained by eliminating a block of size $n$ from $\mu$ if it has one, and otherwise $\Gamma_{\mu-1_{n}}=0$; equivalently, $\theta$ acts as a derivation for $\star$ product and equals $n$ on $\Gamma_{(n)}$. This formula is easy to verify, e.g. by working on the cartesian product and using the projection formula. Then using that $f_{\mu, g}=h_{\mu, g}(0)$, it follows that

$$
h_{\mu, g}(u)=f_{\mu, g}+u \sum_{n} n h_{\mu-1_{n}}(u+n, g)
$$

Because $f_{\mu, g}$ has been computed above and $h_{\emptyset}=1$, this recursion computes all the $h_{\mu, g}(u)$. Explicitly,

$$
h_{\mu, g}(u)=\sum_{\nu \leq \mu}\left(\prod_{\{n \mid \nu(n)>0\}} n^{\nu(n)}(u+(\nu(n)-1) n)\right) f_{\mu-\nu, g}
$$

(sum over all partitions $\nu$ dominated by $\mu$, product over all block sizes $n$ occurring in $\nu)$.

Example 2.39. Assume $B$ is 1-dimensional and the boundary locus corresponding to $\theta$ is a point, so the associated boundary family is a single smooth curve $X^{\theta}$ of 
genus $g-1$. Then using (2.4.8), we get

$$
\begin{aligned}
& \int_{X_{B}^{[m]}}\left(-\Gamma^{(m)}\right)^{m-n+1} F_{j}^{n, m}(\theta) \\
= & \int_{F_{j}^{n, m}(\theta)}\left(-\Gamma^{(m)}\right)^{m-n+1} \\
= & (-1)^{m-n} \sum_{a=0}^{m-n} \int_{\left(X^{\theta}\right)^{m-n}}\left(\Gamma^{(m-n)}+(n-j+1) \theta_{x}+j \theta_{y}\right)^{a} \\
= & (-1)^{m-n}(m-n+1) \int_{\left(X^{\theta}\right)^{m-n}}\left(\Gamma^{(m-n)}+(n+1) \theta_{m-n}\right)^{m-n} \\
= & (-1)^{m-n}(m-n+1) h_{m-n}(n+1, g-1)
\end{aligned}
$$

(see Example 2.34). Note that this number is independent of $j$ for $1 \leq j \leq n-1$. This formula extends easily to the case of a node scroll $F_{j, \mu}^{n, m}$ constrained by a partition $\mu$ of weight $m-n$ and degree $d=d(\mu)=\sum(n-1) \mu(n)$, i.e. $F_{j}^{n, m}(\theta)$ restricted over $\Gamma_{\mu}\left(X^{\theta}\right)$ : we have

$$
\begin{aligned}
& \int_{X_{B}^{[m]}}\left(-\Gamma^{(m)}\right)^{m-n+1-d} F_{j, \mu}^{n, m}(\theta) \\
& =(-1)^{m-d-n}(m-d-n+1) \int_{\Gamma_{\mu}\left(X^{\theta}\right)}\left(\Gamma^{(m-n)}+(n+1) \theta\right)^{m-n-d} \\
& =(-1)^{m-d-n}(m-d-n+1) h_{\mu}(n+1, g-1) .
\end{aligned}
$$

These results are readily combined with Proposition 2.20. To state the result, define, for a partion $\mu$,

$$
N(\mu)=(-1)^{m-d(\mu)+1}(m-d(\mu)) \sum_{\mu(n)>0} \frac{\nu_{n}}{\mu(n)} h_{\mu-1_{n}}(n+1, g-1)
$$

where

$$
\nu_{n}=\frac{n^{2}(n-1)(n+1)}{12}=\sum \frac{j(n-j) n}{2}
$$

and $h$ is as above. Then we obtain, for a good pencil with $\sigma$ singular fibres:

$$
\int_{\Gamma_{\mu}}\left(-\Gamma^{(m)}\right)^{m-d(\mu)}=\int_{\Gamma_{\mu}\left[\operatorname{Dsc}^{(m)}-U_{\omega}\right]}\left(-\Gamma^{(m)}\right)^{m-d(\mu)-1}+\sigma N(\mu) .
$$

Thus, the degree of a diagonal locus decomposes as a sum of a singular contribution plus the degree of a smaller-dimensional (twisted) diagonal locus; of course, the latter terms itself decomposes in a like manner, etc. 
Combining the above examples, we obtain a closed-form formula for the degree of the discriminant polarization in the pencil case:

Proposition 2.40. For a pencil $X / B$ with $\sigma$ singular fibres and canonical degree $\omega^{2}$, the degree of the $m$-th discriminant polarization is

$$
\int_{X_{B}^{[m]}}\left(-\Gamma^{(m)}\right)^{m+1}=(-1)^{m+1} f_{g, m}^{1} \omega^{2}+\sigma \sum_{|\mu|=m}(-1)^{d(\mu)} L(\mu) N(\mu)
$$

where $f_{g, m}^{1}$ is given by (2.4.28), $L(\mu)$ is given by (2.4.29) with $\mu$ viewed as a ppartition with zero exponents, and $N(\mu)$ is given by (2.4.36).

REMARK 2.41. In general, for a pencil $X / B$ (respectively, a single smooth curve over a point) and line bundle $L$ on $X$, the value of

$$
\int_{X_{B}^{[m]}}\left(-\Gamma^{(m)}\right)^{a}[m]_{*}(L)^{b}, \forall a, b, a+b=m+1, b \geq 0,
$$

for fixed $\mathrm{m}$, is a polynomial in $\omega^{2}, L . \omega, L^{2}$, fibre degree $d$, fibre genus $g$, and the number $\sigma$ of singular points, linear in $\sigma$. For a single smooth curve, the analogous number is a polynomial in $d, g$ only. Indeed the latter assertion is clear. For the former, the case $b=0$ has been discussed above. For $b>0$ we use induction on $m$ and the flaglet Hilbert scheme discussed in §3.1. We can write

$$
\begin{aligned}
\int_{X_{B}^{[m]}}\left(-\Gamma^{(m)}\right)^{a}[m]_{*}(L)^{b}= & \frac{1}{m} \int_{X_{B}^{[m, m-1]}} p_{m-1}^{*}\left(\left(-\Gamma^{(m)}\right)^{a}[m]_{*}(L)^{b-1}\right) a^{*}(L) \\
& +p_{m-1}^{*}\left(\left(-\Gamma^{(m)}\right)^{a}[m]_{*}(L)^{b-2}\right) a^{*}\left(L^{2}\right) .
\end{aligned}
$$

The first summand is just $d$ (=fibre degree) times an analogous number for $m-1$, while the second summand, which does not occur for $b=1$, is just $L^{2}$ times an analogous summand for $m-1$ on a general fiber.

Note that the remark implies that the values in question are independent of the distribution of genus or $L$-degrees in reducible fibres. This had been pointed out by Gwoho Liu.

2.5. Tautological module. We are now in position to give the formal (recursive) definition of the tautological module $T^{m}(X / B)$ and the proof of Theorem 2.1.

Definition 2.42. Let $X / B$ be a family of (possibly pointed) nodal curves. Given a (co)homology theory $H_{\bullet}, H^{\bullet}$ admitting a natural map from $A_{\bullet}, A^{\bullet}$ and a $\mathbb{Q}$-subalgebra $R \subset H^{\bullet}(X)_{\mathbb{Q}}$ containing the canonical class $\omega$ and the classes of all marked points, the tautological module $T_{R}^{m}(X / B)$ is the $R$-submodule of $\operatorname{Hom}\left(\operatorname{TS}(R), H_{\bullet}\left(X_{B}^{[m]}\right)\right)$ generated by

$i$. the twisted polyblock diagonal classes $\Gamma_{\mu}[], w(\mu)=m$;

ii. the direct images on $X_{B}^{[m]}$ the twisted node scroll classes $F_{j}^{n}(\theta)[\beta]$ and the twisted node scroll sections $-\Gamma^{(m)} \cdot F_{j}^{n}(\theta)[\beta]$ as $(T, \delta, \theta)$ ranges over a fixed covering system of boundary data for the family $X / B, \beta \in T_{R}^{m-n}\left(X_{T}^{\theta}\right)$ and $2 \leq n \leq m$. 
For the default choice $R=\mathbb{Q}\left[\omega, p_{1}, \ldots, p_{k}\right]$, where $p_{1}, \ldots, p_{k}$ are the markings, $H^{\bullet}=$ $A^{\bullet}, H_{\bullet}=A_{\bullet}$, we denote $T_{R}^{m}$ by $T^{m}$.

Proof of Theorem 2.1. We wish to compute the product of a tautological class $c$ by $\Gamma^{(m)}$. If $c$ is a (twisted) diagonal class $\Gamma_{\mu}[\alpha$.], this is clear from Proposition 2.20. If $c$ is a twisted node scroll class $F_{j}^{n}[\alpha$.$] , it is obvious. Finally if c$ is a node scroll section $-\Gamma^{(m)} \cdot F_{j}^{n}(\theta)[\alpha$.$] , it is clear from the case \ell=2$ of Theorem 2.24 .

REMARK 2.43. It is perhaps advantageous to view $T_{R}^{m}(X / B)$ as a functor on the category of $B$-schemes, associating to a map $T \rightarrow B$ the module $T_{R}^{m}\left(\tilde{X}_{T} / T\right)$, where $\tilde{X}_{T}$ is a desingularization of $X \times_{B} T$. We will not pursue this formally though.

REMARK 2.44. In the important special case of computing a power $\left(\Gamma^{(m)}\right)^{k}$ it is probably more efficient not to proceed by simple recursion, but rather to apply just Proposition 2.20 repeatedly to express $\left(\Gamma^{(m)}\right)^{k}$ in terms of twisted diagonals plus classes $\left(\Gamma^{(m)}\right)^{t} . F$ for various $t$ 's and various $F$ 's; then each of the latter classes can be computed at once using Theorem 2.24.

3. Tautological transfer and Chern numbers. In this chapter we will complete the development of our intersection calculus. First we study the transfer operation $\tau_{m}$, taking cycles on $X_{B}^{[m-1]}$ to cycles (of dimension larger by 1 ) on $X_{B}^{[m]}$, via the flag Hilbert scheme $X_{B}^{[m, m-1]}$. Our main aim is to show that transfer can be effected within the Tautological modules. In the Transfer Theorem 3.4 we will show in fact that for any basic tautological class $u$ on $X_{B}^{[m-1]}$, the image $\tau_{m}(u)$ is a simple linear combination of basic tautological classes on $X_{B}^{[m]}$. We then review a splitting principle established in [22], which expresses the Chern classes of the tautological bundle $\Lambda_{m}(E)$, pulled back on $X_{B}^{[m, m-1]}$, in terms of those of $\Lambda_{m-1}(E)$, the discriminant polarization $\Gamma^{(m)}$, and base classes. Putting this result together with the Module Theorem and the Transfer Theorem yields the calculus for arbitrary polynomials in the Chern classes of $\Lambda_{m}(E)$.

3.1. Flaglet geometry and the transfer theorem. In this section we study the $(m, m-1)$ flag (or 'flaglet') Hilbert scheme, which we view as a correspondence between the Hilbert schemes for lengths $m$ and $m-1$ providing a way of transporting cycles, especially tautological ones, between these Hilbert schemes. We will make strong, chapter-verse use of the results of [23].

Thus let

$$
X_{B}^{[m, m-1]} \subset X^{[m]} \times_{B} X^{[m-1]}
$$

denote the flag Hilbert scheme, parametrizing pairs of schemes $\left(z_{1}, z_{2}\right)$ satisfying $z_{1} \supset$ $z_{2}$. This comes equipped with a (flag) cycle map

$$
c_{m, m-1}: X_{B}^{[m, m-1]} \rightarrow X_{B}^{(m, m-1)},
$$

where $X_{B}^{(m, m-1)} \subset X_{B}^{(m)} \times_{B} X_{B}^{(m-1)}$ is the subvariety parametrizing cycle pairs $\left(c_{m} \geq\right.$ $\left.c_{m-1}\right)$. Note that the normalization of $X_{B}^{(m, m-1)}$ may be identified with $X_{B}^{(m-1)} \times_{B} X$; however the normalization map, though bijective, is not an isomorphism. Note also that we also have an ordered version $X_{B}^{\lceil m, m-1\rceil}$, with its own cycle map

$$
o c_{m, m-1}: X_{B}^{\lceil m, m-1\rceil} \rightarrow X_{B}^{m} .
$$


In addition to the obvious projections

$$
X_{B}^{[m]} p_{m} \swarrow X_{B}^{[m, m-1]} \searrow p_{m-1} X_{B}^{[m-1]}
$$

with respective generic fibres $m$ distinct points (corresponding to removing a point from a given $m$-tuple) and a generic fibre of $X / B$ (corresponding to adding a point to a given $m-1$-tuple), $X_{B}^{[m, m-1]}$ admits a natural map

$$
\begin{gathered}
a: X_{B}^{[m, m-1]} \rightarrow X, \\
\left(z_{1} \supset z_{2}\right) \mapsto \operatorname{ann}\left(z_{1} / z_{2}\right)
\end{gathered}
$$

(identifying $X$ with the Hilbert scheme of colength-1 ideals). Therefore $X_{B}^{[m, m-1]}$ admits a 'refined cycle map' (factoring the flag cycle map)

$$
\begin{gathered}
c: X_{B}^{[m, m-1]} \rightarrow X \times_{B} X_{B}^{(m-1)} \\
c=a \times\left(c_{m-1} \circ p_{m-1}\right) .
\end{gathered}
$$

Now in [23] (Theorem 5 et seq., especially Construction 5.4 p.442) we worked out a complete model for $X_{B}^{[m, m-1]}$, locally over $X_{B}^{(m, m-1)}$. Let

$$
\begin{gathered}
H_{m} \subset X_{B}^{(m)} \times \tilde{C}_{[u, v .]}^{m} \subset X_{B}^{(m)} \times \mathbb{P}_{Z .}^{m-1}, \\
H_{m-1} \subset X_{B}^{(m-1)} \times \tilde{C}_{\left[u^{\prime}, v^{\prime} \cdot\right]}^{m-1} \subset X_{B}^{(m-1)} \times \mathbb{P}_{Z^{\prime}}^{m-2}
\end{gathered}
$$

be respective local models for $X_{B}^{[m]}, X_{B}^{[m-1]}$ as constructed in $\S 1$ above, with coordinates as indicated. Consider the subscheme

$$
H_{m, m-1} \subset H_{m} \times_{B} H_{m-1} \times_{X_{B}^{(m)} \times X_{B}^{(m-1)}} X_{B}^{(m, m-1)}
$$

defined by the equations

$$
u_{i}^{\prime} v_{i}=\left(\sigma_{1}^{x}-\sigma_{1}^{\prime x}\right) u_{i} v_{i}^{\prime}, \quad v_{i}^{\prime} u_{i+1}=\left(\sigma_{1}^{y}-\sigma_{1}^{\prime y}\right) v_{i+1} u_{i}^{\prime}, \quad 1 \leq i \leq m-2
$$

or alternatively, in terms of the $Z$ coordinates,

$$
\begin{aligned}
Z_{i} Z_{j}^{\prime} & =\left(\sigma_{1}^{x}-\sigma_{1}^{\prime}\right) Z_{i+1} Z_{j-1}^{\prime}, \quad i+1 \leq j \leq m-1 \\
& =\left(\sigma_{1}^{y}-\sigma_{1}^{\prime}\right) Z_{i-1} Z_{j+1}^{\prime}, l \quad 1 \leq j \leq i-2 .
\end{aligned}
$$

To 'explain' these relations in part, note that in the ordered model over $X_{B}^{m}$, we have

$$
\sigma_{1}^{x}-\sigma_{1}^{\prime x}=x_{m}, \sigma_{1}^{y}-\sigma_{1}^{\prime y}=y_{m}
$$

and then the analogue of (3.1.8) for the $G$ functions is immediate from the definition of these in [19], §4. Then the result of [23], Thm. 5, is that $H_{m, m-1}$, with its map to $X_{B}^{(m, m-1)}$ is isomorphic to a neighborhood of the special fibre over $(m p,(m-1) p)$ of 
the flag Hilbert scheme $X_{B}^{[m, m-1]}$. In fact the result of [23] is even more precise and identifies $H_{m, m-1}$ with a subscheme of $H_{m} \times{ }_{B} H_{m-1}$ and even of $H_{m-1} \times{ }_{B} \tilde{C}^{m} \times_{B} X$, where the map to $X$ is the annihilator map $a$ above.

As noted in [23], Thm 5, the special fibre of the flag cycle map on $H_{m, m-1}$, aka the punctual flag Hilbert scheme, is a normal-crossing chain of $\mathbb{P}^{1}$ 's:

$$
C^{m, m-1}=\tilde{C}_{1}^{m} \cup \tilde{C}_{1}^{m-1} \cup \tilde{C}_{2}^{m} \cup \ldots \cup \tilde{C}_{m-2}^{m-1} \cup \tilde{C}_{m-1}^{m} \subset C^{m} \times C^{m-1},
$$

where the embedding is via

$$
\tilde{C}_{i}^{m} \rightarrow C_{i}^{m} \times\left\{Q_{i}^{m-1}\right\}, \quad \tilde{C}_{i}^{m-1} \rightarrow\left\{Q_{i+1}^{m}\right\} \times C_{i}^{m-1}
$$

and in particular,

$$
\tilde{C}_{i}^{m} \cap \tilde{C}_{i}^{m-1}=\left\{\left(Q_{i+1}^{m}, Q_{i}^{m-1}\right)\right\}, \tilde{C}_{i}^{m-1} \cap \tilde{C}_{i+1}^{m}=\left\{\left(Q_{i+1}^{m}, Q_{i+1}^{m-1}\right)\right\}
$$

where $Q_{i}^{m}=\left(x^{m-i+1}, y^{i}\right)$ as usual.

TheOREM 3.1. The cycle map $c_{m, m-1}$ exhibits the flag Hilbert scheme $X_{B}^{[m, m-1]}$ as the blow-up of the sheaf of ideals $\mathcal{I}_{D^{m, m-1}}:=\mathcal{I}_{D^{m-1}} \cdot \mathcal{I}_{D^{m}}$ on $X_{B}^{(m, m-1)}$.

We shall not really need this result, just the explicit constructions above, so we just sketch the proof, which is analogous to that of the Blowup Theorem of [19]. To begin with, it is again sufficient to prove the ordered analogue of this result, for the 'ordered flag cycle map'

$$
X_{B}^{\lceil m, m-1\rceil} \rightarrow X_{B}^{m}
$$

Here $X_{B}^{\lceil m, m-1\rceil}$ is embedded as a subscheme of $X_{B}^{\lceil m\rceil} \times_{X_{B}^{m}}\left(X_{B}^{\lceil m-1\rceil} \times_{B} X\right)$, and we have already observed that as such, it satisfies the equations (3.1.8).

Now we will use the following construction. Let $\mathcal{I}_{1}, \mathcal{I}_{2}$ be ideals on a scheme $Y$. Then the surjection of graded algebras

$$
\left(\bigoplus_{n} \mathcal{I}_{1}^{n}\right) \otimes\left(\bigoplus_{n} \mathcal{I}_{2}^{n}\right) \rightarrow \bigoplus_{n}\left(\mathcal{I}_{1} \mathcal{I}_{2}\right)^{n}
$$

yields a closed immersion

$$
\mathcal{B} \ell_{\mathcal{I}_{1} \mathcal{I}_{2}} Y \hookrightarrow \mathcal{B} \ell_{\mathcal{I}_{1}} Y \times_{Y} \mathcal{B} \ell_{\mathcal{I}_{2}} Y
$$

the latter is in turn a subscheme of the Segre subscheme

$$
\mathbb{P}\left(\mathcal{I}_{1}\right) \times_{Y} \mathbb{P}\left(\mathcal{I}_{2}\right) \subset \mathbb{P}\left(\mathcal{I}_{1} \otimes \mathcal{I}_{2}\right)
$$

In our case, the Blowup Theorem of [19] allows us to identify

$$
O H_{m} \simeq \mathcal{B} \ell_{\mathcal{I}_{O D^{m}}} X_{B}^{m}, O H_{m-1} \times_{B} X \simeq \mathcal{B} \ell_{\mathcal{I}_{O D^{m-1} . X_{B}^{m}}} X_{B}^{m}
$$

(where $O H_{m}=H_{m} \times_{X_{B}^{(m)}} X_{B}^{m}$ etc.), whence an embedding

$$
\mathcal{B} \ell_{\mathcal{I}_{O D^{m, m-1}}} X_{B}^{m} \rightarrow O H_{m} \times_{X_{B}^{m}}\left(O H_{m-1} \times_{B} X\right) .
$$

As observed above, the generators $G_{i} \cdot G_{j}^{\prime}$ satisfy the analogues of the relations (3.1.8), so the image is actually contained in $O H_{m, m-1}$, so we have an embedding

$$
\mathcal{B} \ell_{\mathcal{I}_{O D^{m}, m-1}} X_{B}^{m} \rightarrow O H_{m, m-1} \text {. }
$$


We are claiming that this is an isomorphism. This can be verified locally, as in the proof of the Blowup Theorem in [19].

One consequence of the explicit local model for $X_{B}^{[m, m-1]}$ is the following

COROllary 3.2 .

i. The projection $q_{m-1}$ is flat, with 1-dimensional fibres;

ii. Let $z \in X_{B}^{[m-1]}$ be a subscheme of a fibre $X_{s}$, and let $z_{0}$ be the part of $z$ supported on nodes of $X_{s}$, if any. Then if $z_{0}$ is principal (i.e. Cartier) on $X_{s}$, the fibre $q_{m-1}^{-1}(z)$ is birational to $X_{s}$ and its general members are equal to $z_{0}$ locally at the nodes.

Proof. (i) is proven in [22], and also follows easily from our explicit model $H_{m, m-1}$. As for (ii), we may suppose, in the notation of [23], that $z_{0}$ is of type $I_{i}^{n}(a)$. Now if $z^{\prime} \in q_{m-1}^{-1}(z)$, then the part $z_{0}^{\prime}$ of $z^{\prime}$ supported on nodes must have length $n$ or $n+1$. In the former case $z_{0}^{\prime}=z_{0}$, while in the latter case $z_{0}^{\prime}$ must equal $Q_{i+1}^{n+1}$ by [23], Thm. 5 p. 438 , in which case $z^{\prime}$ is unique, hence not general.

Another consequence of the Theorem is a description of the 'flaglet' small diagonal. Denote by

$$
\tilde{\Gamma}_{(m)} \subset X^{[m, m-1]}
$$

the inverse image of the small diagonal $\Gamma_{(m)} \subset X_{B}^{[m]}$. In Proposition 2.2 we described the usual small diagonal as an explicit blowup. Thanks to the Theorem, we obtain an analogous description for the flaglet small diagonal:

Corollary 3.3. The cycle map $\tilde{\Gamma}_{(m)} \rightarrow X$, is the blowing up of the ideal $J_{m}^{\theta \cdot} J_{m-1}^{\theta \cdot}(c f .(2.1 .2))$.

See $\S 3.3$ for further discussion of the flaglet small diagonal and the associated 'punctual' transfer operation.

Next we define the fundamental transfer operation. Essentially, this takes cycles from $X_{B}^{[m-1]}$ to $X_{B}^{[m]}$, but we also allow the additional flexibility of twisting by base classes via the $m$-th factor. Thus the twisted transfer map $\tau_{m}$ is defined by

$$
\begin{gathered}
\tau_{m}=\tau_{m, X / B}: A .\left(X_{B}^{[m-1]}\right) \otimes A .(X) \rightarrow A .\left(X_{B}^{[m]}\right)_{\mathbb{Q}}, \\
\tau_{m}=q_{m *}\left(q_{m-1}^{*} \otimes a^{*}\right) .
\end{gathered}
$$

Note that this operation raises dimension by 1 and preserves codimension. Suggestively, and a little abusively, we will write a typical decomposable element of the source of $\tau_{m}$ as $\gamma \beta_{(m)}$ where $\gamma \in A .\left(X_{B}^{[m-1]}\right), \beta \in A$. $(X)$. Note also that when $B$ is a point (and $X$ is smooth), $\tau_{m}$ is just the 'identity' in the above notation.

The following Transfer Theorem, which computes the transfer $\tau_{m}$ on the Tautological Module, is a key to our inductive computation of Chern numbers. The formula involves, in the case of node classes, the analogous transfer operation in lower degree on boundary families $X_{T}^{\theta}$, and may be viewed as a commutation relation between the transfer operator and the node scroll and node section operators. The relation involves the operators $e_{j}^{n, m}$ of (2.4.5).

Theorem 3.4. (Tautological transfer) $\tau_{m}$ takes tautological classes on $X_{B}^{[m-1]}$ to tautological classes on $X_{B}^{[m]}$. More specifically we have, for any class $\beta \in A .(X)$ : 
i. for any twisted polyblock diagonal class $\Gamma_{\mu}[\alpha],. \alpha \in \operatorname{TS}_{\mu}(H \cdot(X)), w(\mu)=m-$ 1 ,

$$
\tau_{m}\left(\Gamma_{\mu}[\alpha .] \beta_{(m)}\right)=\Gamma_{\mu+1_{1}}[\alpha . \beta]
$$

where $1_{1}$ is the distribution of weight 1 and support $\{1\}$ and $\alpha . \beta$ is the formal symmetric multiplication;

ii. for any twisted node scroll class $F_{j}^{n, m-1}(\theta)[\alpha], \alpha \in T^{m-n-1}\left(X_{T}^{\theta}\right)$,

$$
\tau_{m}\left(F_{j}^{n, m-1}(\theta)[\alpha] \beta_{(m)}\right)=F_{j}^{n, m}(\theta)\left[\tau_{m-n, X_{T}^{\theta} / T}\left(\alpha \otimes\left(\left.\beta\right|_{X_{T}^{\theta}}\right)\right]\right.
$$

iii. for any twisted node section $-\Gamma^{(m-1)} \cdot F_{j}^{n, m-1}(\theta)[\alpha]$,

$$
\begin{aligned}
& \tau_{m}\left(-\Gamma^{(m-1)} F_{j}^{n, m-1}(\theta)[\alpha] \beta_{(m)}\right) \\
= & \theta^{*}(\beta) F_{j}^{n+1, m}(\theta)[\alpha]+\left(-\Gamma^{(m)}\right) F_{j}^{n, m}(\theta)\left[\tau_{m-n, X_{T}^{\theta} / T}\left(\left.\alpha \cdot \beta\right|_{X_{T}^{\theta}}\right)\right. \\
& -F_{j}^{n, m}(\theta)\left[e_{j+1}^{n, m}(\theta)\left(\tau_{m-n, X_{T}^{\theta} / T}\left(\left.\alpha \cdot \beta\right|_{X_{T}^{\theta}}\right)\right)\right] \\
& +F_{j}^{n, m}(\theta)\left[\tau_{m-n, X_{T}^{\theta} / T}\left(\left.e_{j+1}^{n, m-1}(\theta)(\alpha) \cdot \beta\right|_{X_{T}^{\theta}}\right)\right] .
\end{aligned}
$$

Proof. Part (i) is obvious. As for Part (ii), the flatness of $q_{m-1}$ allows us to work over a general $z \in F$ and then Corollary 3.2, (ii) allows us to assume that the added point is a general point on the fibre $X_{s}$, which leads to (3.1.17).

As for (iii), we recall Corollary 8.4 of [19], which states (using our current notation, not consistent with notation there) that on $F_{j}^{n, m-1}(\theta)$, if we denote by $Q_{j}^{n, m-1}$ the canonical cross-section $\mathbb{P}\left(D_{j}^{n, m-1}\right)$, and by $\Gamma^{(m-n-1)}$ the pullback of the discriminant from $\left(X^{\theta}\right)_{T}^{[m-n-1]}$, then we have

$-\Gamma^{(m-1)}+\Gamma^{(m-n-1)} \sim Q_{j}^{n, m-1}+p_{[m-n-1]}^{*}\left(D_{j+1}^{n, m-1}\right) \sim Q_{j+1}^{n, m-1}+p_{[m-n-1]}^{*}\left(D_{j}^{n, m-1}\right)$.

Hence,

$$
-\Gamma^{(m-1)} \sim Q_{j}^{n, m-1}+e_{j+1}^{n, m-1} .
$$

Similarly, on $F_{j}^{n, m}(\theta)$, we have

$$
-\Gamma^{(m)}+\Gamma^{(m-n)} \sim Q_{j}^{n, m}+p_{[m-n]}^{*}\left(D_{j+1}^{n, m}\right) \sim Q_{j+1}^{n, m}+p_{[m-n]}^{*}\left(D_{j}^{n, m}\right),
$$

hence

$$
-\Gamma^{(m)} \sim Q_{j}^{n, m}+e_{j+1}^{n, m} \sim Q_{j+1}^{n, m}+e_{j}^{n, m} .
$$

Therefore, it will suffice to prove that

$$
\tau_{m}\left(Q_{j+1}^{n, m-1}[\alpha] \beta_{(m)}\right)=\theta^{*}(\beta) F_{j+1}^{n+1, m}[\alpha]+Q_{j+1}^{n, m}[\alpha \beta]
$$

and similarly for $j$, which case is similar (see below). It will suffice to prove this without the $\alpha, \beta$ twisting.

To this end, note that, with $Q=Q_{j+1}^{n, m-1}, q_{m-1}^{*} Q$ splits in two parts, depending on whether the point $w$ added to a scheme $z \in Q$ is in the off-node or nodebound 
portion of $z$. It is easy to see that the first part gives rise to the 2 nd term in the RHS of (3.1.19).

The analysis of the other part, which leads to the first summand in the RHS of (3.1.19) is a bit more involved. In substance, what has to be proved in the case at hand is that $F_{j+1}^{n+1, m}$ appears with coefficient equal to 1 . To begin with, it is easy to see that we may assume $m=n+1$, in which case $F$ is just a $\mathbb{P}^{1}$, namely $C_{j}^{m-1}$. Now referring to (3.1.9), the nodebound portion of $q_{m-1}^{-1}\left(C_{j}^{m-1}\right)$, as a set, is given by $\tilde{C}_{j}^{m} \cup \tilde{C}_{j}^{m-1} \cup \tilde{C}_{j+1}^{m}$ and that of $q_{m-1}^{-1}(Q)$ is $\tilde{C}_{j+1}^{m}$. It will now suffice to show that the 1-dimensional cycle $q_{m-1}^{-1}(Q)$ contains $\tilde{C}_{j+1}^{m}$ with multiplicity 1 .

The latter assertion will be an elementary consequence of the equations on $\mathrm{p}$. 440, l. 9-14 of [23], describing the local model $H_{m, m-1}$, as well as those on p. 433, describing the analogous local model $H_{m}$, to which equations we will be referring constantly in the remainder of the present proof. Note that $c_{m-i}$ (resp. $b_{i-1}^{\prime}$ ) plays the role of the affine coordinate $u_{i} / v_{i}$ (resp. $v_{i-1}^{\prime} / u_{i-1}^{\prime}$ ). Also our $j+1$ is the $i$ there. We work on $q_{m-1}^{-1}\left(C_{j}^{m-1}\right)$. Now to complete the proof, it will suffice to prove

Claim : In a neighborhood of the point

$$
\left(Q, Q_{j+1}^{m}\right)=\left(Q_{j+1}^{m-1}, Q_{j+1}^{m}\right) \in \tilde{C}_{j}^{m-1} \cap \tilde{C}_{j+1}^{m} \subset X_{B}^{[m, m-1]},
$$

$q_{m-1}^{-1}(Q)$ contains $\tilde{C}_{j+1}^{m}$ with multiplicity 1 .

To see this note that the defining equations of $C_{j}^{m-1}$ on $X_{B}^{[m-1]}$ are given by setting all $a_{k}^{\prime}$ and $d_{k}^{\prime}$, as well as $c_{m-i-1}^{\prime}$ to zero . By loc. cit. p.433 1.9, this implies that we have $b_{1}^{\prime}=\ldots=b_{i-2}^{\prime}=0$ on $q_{m-1}^{-1}\left(C_{j}^{m-1}\right)$ as well. At a general point of $C_{j+1}^{m}$, $c_{m-i}$ is nonzero. Therefore we may consider $c_{m-i}$ as a unit. By loc. cit. p.440, eq. (15), we conclude $a_{m-i}=0$. From this we see easily that all $a_{k}=d_{k}=0$ except $d_{i-1}$, which is a local equation for $\tilde{C}_{j+1}^{m}$, while $b_{i-1}^{\prime}$ is a coordinate along $C_{j}^{m-1}$ having $Q_{j+1}^{m-1}$ as its unique zero. Now by p.440 l. $14, b_{i-1}^{\prime}$ and $d_{i-1}$ differ by the multiplicative unit $-c_{m-i}$, therefore $b_{i-1}^{\prime}$ cuts out $\tilde{C}_{j+1}^{m}$ with multiplicity 1 , which proves our Claim. $\square$

For many purposes, it is possible and even more convenient to use (3.1.19) directly, rather than through Theorem3.4, to compute the transfer on node sections. In particular, this form works better with iterated node scroll $/ Q$-sections. To state the result, it is convenient to introduce the following notation for the iterated scroll $/ Q$-sections:

$$
\begin{gathered}
(F Q)_{j_{1}, \ldots, j_{r} ; j_{1}^{\prime}, \ldots, j_{s}^{\prime}}^{n_{1}, \ldots, n_{r} ; n_{1}^{\prime}, \ldots, n_{s}^{\prime} ; m}\left(\theta_{1}, \ldots, \theta_{r} ; \theta_{1}^{\prime}, \ldots, \theta_{s}^{\prime}\right)[\alpha] \\
:=F_{j_{1}}^{n_{1}, m}\left(\theta_{1}\right)\left[\ldots\left[Q_{j_{1}^{\prime}}^{n_{1}^{\prime}, m-n_{1}-\ldots-n_{r}}\left(\theta_{1}^{\prime}\right) \ldots[\alpha] \ldots\right]\right.
\end{gathered}
$$

where $\theta_{1}, \ldots, \theta_{s}^{\prime}$ is a collection of distinct nodes. Then we have

\section{Corollary 3.5 .}

$$
\begin{aligned}
& \tau_{m}\left((F Q)_{j_{1}, \ldots, j_{r} ; j_{1}^{\prime}, \ldots, j_{s}^{\prime}}^{n_{1}, \ldots, n_{s} ; n_{1}^{\prime}, \ldots, n_{s}^{\prime} ; m-1}\left(\theta_{1}, \ldots, \theta_{r} ; \theta_{1}^{\prime}, \ldots, \theta_{s}^{\prime}\right)[\alpha] \beta_{m}\right) \\
& =(F Q)_{j_{1}, \ldots, j_{r} ; j_{1}^{\prime}, \ldots, j_{s}^{\prime}}^{n_{1}, \ldots, n_{r} ; n^{\prime}, \ldots, n_{s}^{\prime} ; m}\left(\theta_{1}, \ldots, \theta_{r} ; \theta_{1}^{\prime}, \ldots, \theta_{s}^{\prime}\right)\left[\tau_{m-|n .|-\left|n^{\prime} .\right|}(\alpha) \beta_{m}\right] \\
& +\sum_{i=1}^{s}\left(\theta_{i}^{\prime}\right)^{*}(\beta)(F Q)^{n_{1}, \ldots, n_{r}, n_{i}^{\prime}+1 ; n_{1}^{\prime}, \ldots, \hat{n}_{i}^{\prime}, \ldots, n_{s}^{\prime} ; m} \\
& \times\left(\theta_{1}, \ldots, \theta_{r}, \theta_{i}^{\prime} ; \theta_{1}^{\prime}, \ldots, \hat{\theta}_{i}^{\prime}, \ldots, \theta_{s}^{\prime}\right)\left[\tau_{m-|n .|-\left|n^{\prime} .\right|}(\alpha) \beta_{m}\right] .
\end{aligned}
$$


3.2. Transfer and Chern numbers. We are now ready to tackle the computation of Chern numbers, and in fact all polynomials in the Chern classes of the tautological bundle on the relative Hilbert scheme $X_{B}^{[m]}$. The computation is based on passing from $X_{B}^{[m]}$ to the corresponding full-flag Hilbert scheme $W=W^{m}(X / B)$ studied in [22] and a diagonalization theorem for the total Chern class of (the pullback of) a tautological bundle on $W$, expressing it either as a simple (factorable) polynomial in diagonal classes induced from the various $X_{B}^{[n]}, n \leq m$, plus base classes, or, more conveniently, as the product of the Chern class of a smaller tautological bundle and a diagonal class. Given this, we can compute Chern numbers essentially by repeatedly applying the transfer calculus of the last section.

We start by reviewing some results from [22]. Let

$$
W^{m}=W^{m}(X / B) \stackrel{\pi^{(m)}}{\longrightarrow} B
$$

denote the relative flag-Hilbert scheme of $X / B$, parametrizing flags of subschemes

$$
z .=\left(z_{1}<\ldots<z_{m}\right)
$$

where $z_{i}$ has length $i$ and $z_{m}$ is contained in some fibre of $X / B$. Let

$$
w^{m}: W^{m} \rightarrow X_{B}^{[m]}, w^{m, i}: W^{m} \rightarrow X_{B}^{[i]}
$$

be the canonical (forgetful) maps. Let

$$
a_{i}: W^{m} \rightarrow X
$$

be the canonical map sending a flag $z$. to the 1-point support of $z_{i} / z_{i-1}$ and

$$
a^{m}=\prod a_{i}: W^{m} \rightarrow X_{B}^{m}
$$

their (fibred) product, which might be called the 'ordered cycle map'. Let

$$
\mathcal{I}_{m}<\mathcal{O}_{X_{B}^{[m]} \times_{B} X}
$$

be the universal ideal of colength $m$. For any coherent sheaf on $X$, set

$$
\Lambda_{m}(E)=p_{X_{B}^{[m]} *}\left(p_{X}^{*}(E) \otimes\left(\mathcal{O}_{X_{B}^{[m]} \times_{B} X} / \mathcal{I}_{m}\right)\right)
$$

These are called the tautological sheaves associated to $E$; they are locally free if $E$ is. Abusing notation, we will also denote by $\Lambda_{m}(E)$ the pullback of the tautological sheaf to appropriate flag Hilbert schemes mapping naturally to $X_{B}^{[m]}$, such as $W^{m}$ or $X_{B}^{[m, m-1]}$. With a similar convention, set

$$
\Delta^{(m)}=\Gamma^{(m)}-\Gamma^{(m-1)}
$$

(recall that $\Gamma^{(m)}$ is half the 'physical' discriminant and becomes effective and reduced on $W^{m}$; thus $\Delta^{(m)}$ is an effective (integral) divisor). The various tautological sheaves form a flag of quotients on $W^{m}$ :

$$
\ldots \Lambda_{m . i}(E) \rightarrow \Lambda_{m, i-1}(E) \rightarrow \ldots
$$


This flag makes possible a simple formula for the total Chern class of the tautological bundles, namely the following diagonalization theorem ( [22], Cor. 3.2):

TheOREM 3.6. The total Chern class of the tautological bundle $\Lambda_{m}(E)$ pulled back to $W^{3}(X / B)$ is given by

$$
c\left(\Lambda_{m}(E)\right)=\prod_{i=1}^{m} c\left(a_{i}^{*}(E)\left(-\Delta^{(i)}\right)\right) .
$$

An analogue of this, more useful for our purposed, holds already on the flaglet Hilbert scheme. It can be proved in the same way, or as an easy consequence of Thm 3.6

Corollary 3.7. We have an identity in $A \cdot\left(X_{B}^{[m, m-1]}\right)_{\mathbb{Q}}$ :

$$
c\left(\Lambda_{m}(E)\right)=c\left(\Lambda_{m-1}(E)\right) c\left(a_{m}^{*}(E)\left(-\Delta^{(m)}\right)\right) .
$$

Proof. By Theorem 3.6, the RHS and LHS pull back to the same class in $W^{m}$. As the projection $W^{m} \rightarrow X_{B}^{[m, m-1]}$ is generically finite, they agree mod torsion.

REMARK 3.8. If $E$ is a line bundle, then it is easy to see from Theorem 3.6 that

$$
c_{1}\left(\Lambda_{m}(E)\right)=[m]_{*} c_{1}(E)-\Gamma^{(m)}=\Gamma_{1}\left[c_{1}(E)\right]-\Gamma^{(m)} .
$$

EXAMPLE 3.9. On $W^{3}$ :

$$
\begin{aligned}
& c\left(\Lambda_{3}(L)\right)=1+L_{1}+L_{2}+L_{3}-\Gamma^{(3)} \\
& +L_{1} L_{2}+L_{1} L_{3}+L_{2} L_{3}-\left(\Gamma^{(2)}\right)^{2}+\Gamma^{(2)} \Gamma^{(3)}+\Gamma^{(2)} L_{2}-\Gamma^{(2)} L_{3}-\Gamma^{(3)} L_{1}-\Gamma^{(3)} L_{2} \\
& +L_{1} L_{2} L_{3}-\Gamma^{(2)} L_{1} L_{3}-\Gamma^{(3)} L_{1} L_{2}+\Gamma^{(2)} L_{1} L_{2}+\Gamma^{(2)} \Gamma^{(3)} L_{1}-\left(\Gamma^{(2)}\right)^{2} L_{1} .
\end{aligned}
$$

More generally, again as a consequence of Theorem 3.6, we obtain an explicit formula for the Chern classes of $\Lambda_{m}(L)$ on $X_{B}^{[m]}$, for a line bundle $L$. First some notation. For a distribution $\mu$, set

$$
\chi(\mu)=\prod_{n}((n-1) !)^{\mu(n)} .
$$

Corollary 3.10. For a line bundle L, we have

$$
c\left(\Lambda_{m}(L)\right)=\sum_{|\mu| \leq m}(-1)^{|\mu|-\ell(\mu) \mid} \frac{a(\mu) \chi(\mu)}{|\mu| !(m-|\mu|) !} \Gamma_{\mu}\left[(1+[L])^{(\mu)}\right] .
$$

Proof. Straightforward from Theorem 3.6, see [21], Theorem 4.2 (though the formula there is slightly misstated).

REMARK 3.11. In the classical situation of a single smooth curve over a point, multiplying diagonal classes is elementary. For example, in the case of single-block classes, we have

$$
\Gamma_{(n)} \cdot \Gamma_{(\ell)}=2\left(\begin{array}{l}
n \\
2
\end{array}\right)\left(\begin{array}{l}
\ell \\
2
\end{array}\right) \Gamma_{(n+l-2)}[-\omega]+n \ell \Gamma_{(n+\ell-1)}+\left(1+\delta_{n, \ell}\right) \Gamma_{(n \mid \ell)} .
$$


Moreover in the notation of Remark 1.4, we have

$$
\Gamma_{(n+l-2)}[-\omega]=(2-2 g) \theta^{n+\ell-2} .
$$

This allows computation, modulo the combinatorics, of all Chern polynomials of $\Lambda_{m}(L)$. However, Macdonald's formulation, which expresses everything as polynomials of $\Gamma$ and $\theta$, is probably more efficient in this case (no singularities).

EXAMPLE 3.12. In the case of a good pencil, we can give an explicit formula for the polyblock (singularity-free) portion of $\left(\Delta^{(m)}\right)^{k} \Gamma_{\mu}$ in $X_{B}^{[m, m-1]}$, as follows. Define for a partition $\mu=\left(n_{1}, n_{2}, \ldots\right)$,

$$
f_{k}(\mu)=\left(\sum n_{i}\right)^{k}-\sum_{j}\left(\sum_{i \neq j} n_{i}\right)^{k}+\sum_{j<j^{\prime}}\left(\sum_{i \neq j, j^{\prime}} n_{i}\right)^{k} \pm \ldots
$$

and note that this can be identified with the sum of all terms $\frac{k !}{\alpha_{1} ! \ldots \alpha_{\ell} !} n_{1}^{\alpha_{1}} \ldots n_{\ell}^{\alpha_{\ell}}$ where each $\alpha_{i}$ is $>0$. Then

$$
\left(\Delta^{(m)}\right)^{k} \cdot \Gamma_{\mu} \equiv \sum_{\mu=\mu_{1}+\mu_{2}} f_{k}\left(\mu_{2}\right) \Gamma_{\mu_{1}} \star \Gamma_{\left|\mu_{2}\right|+1}\left[(-\omega)^{m-\ell\left(\mu_{2}\right)+1}\right] \quad \text { mod node classes }
$$

To see this expand $\left(\Delta^{(m)}\right)^{k}$ as a multinomial in the $D_{i, m}$ and for each monomial $M$ break up $\mu$ as $\mu_{1}+\mu_{2}$ where no block (resp. every block) of $\mu_{1}$ (resp. $\mu_{2}$ ) has an element occurring in $M$. The product of $\Gamma_{\mu_{2}}$ with $M$ yields $\Gamma_{\left|\mu_{2}\right|+1}\left[(-\omega)^{m-\ell\left(\mu_{2}\right)+1}\right]$, whence the result.

Motivated by the Corollary we make the following definition.

Definition 3.13. Let $R$ be a $\mathbb{Q}$-subalgebra of $A(X)$ containing 1 , the canonical class $\omega$ and the classes of all the marked points. The Chern tautological ring on $X_{B}^{[m]}$, denoted

$$
T C_{R}^{m}=T C_{R}^{m}(X / B),
$$

is the R-subalgebra of $A\left(X_{B}^{[m]}\right)_{\mathbb{Q}}$ generated by the Chern classes of $\Lambda_{m}(E)$ and the discriminant class $\Gamma^{(m)}$

The following is the main result of this paper.

THEOREM 3.14. There is a computable inclusion

$$
T C_{R}^{m} \rightarrow T_{R}^{m} .
$$

More explicitly, any polynomial in the Chern classes of $\Lambda_{m}(E)$, in particular the Chern numbers, can be computably expressed as a linear combination of standard tautological classes: twisted diagonal classes, twisted node scrolls, and twisted node sections.

Proof. For $m=1$ the statement is essentially vacuous. For $m=2$ it is a consequence of the Module Theorem 2.1. For general $m$, we assume inductively the result is true for $m-1$. Given any polynomial $P$ in the Chern classes of $\Lambda_{m}(E)$, Corollary 3.7 implies that we can write its pullback on $X_{B}^{[m, m-1]}$ as a sum of terms of the form $p_{X_{B}^{[m-1]}}^{*}(Q) .\left(\Gamma^{(m)}\right)^{k} . S$ where $Q \in T C_{R}^{m-1}$. By induction, $Q \in T_{R}^{m-1}$, so by the Transfer Theorem 3.4, $\tau_{m}(Q) \in T_{R}^{m}$. By the projection formula and the Module Theorem 2.1, it follows that $P \in T_{R}^{m}$.

REMARK 3.15. This result suggests the natural question: is $T_{R}^{m}$ a ring? more ambitiously, is the inclusion $T C_{R}^{m} \rightarrow T_{R}^{m}$ an equality? 
3.3. Punctual transfer and Plücker formulas. There is a useful variant of the transfer operation for punctual schemes, i.e. those supported at a single point, which are parametrized by the small diagonal $\Gamma_{(m)} \subset X_{B}^{[m]}$. This yields a quicker way to compute Chern classes and Chern numbers of tautological bundles over the small diagonal (compared with computing the analogous objects over the full Hilbert scheme and restricting). Working with the smaller-dimensional $\Gamma_{(m)}$ allows us to obtain geometrically meaningful numbers from the Chern and Segre classes themselves without considering higher-degree polynomials, resulting in some Pluc̈ker-type formulas which, unlike in the case of the full Hilbert scheme, we are able to give in closed form. Note that unlike the Hilbert scheme $X_{B}^{[m]}$, the small diagonal $\Gamma_{(m)}$ is generally singular.

This transfer is based on the correspondence

$$
\Gamma_{(m)} \swarrow^{\tilde{\Gamma}_{(m)}} \searrow{ }_{(m-1)}
$$

where the punctual flaglet Hilbert scheme $\tilde{\Gamma}_{(m)}$ is defined by the Cartesian diagram

$$
\begin{array}{ccc}
\tilde{\Gamma}_{(m)} & \rightarrow & X_{B}^{[m, m-1]} \\
\downarrow & & \downarrow \\
\Gamma_{(m)} & \rightarrow & X_{B}^{[m]}
\end{array} .
$$

As in $\S 2.1$ and Corollary $3.3, \tilde{\Gamma}_{(m)}$ can be identified locally over each node with the unique dominant component of the fibre product over $X$

$$
\prod_{i=1}^{m-1}\left(X_{m, i} / X\right) \times_{X} \prod_{i=1}^{m-2}\left(X_{m-1, i} / X\right)
$$

where each $X_{n, i}$ is the blowup of the ideal $\left(x^{n-i}, y^{i}\right)$. We denote by

$$
\tau_{m}^{0}: A \cdot\left(\Gamma_{(m-1)}\right) \rightarrow A \cdot\left(\Gamma_{(m)}\right)
$$

the induced map, i.e. $p_{[m] *} p_{[m-1]}^{*}$ from Chow cohomology. Also, let $C_{i}^{m}(\theta), C_{i}^{m-1}(\theta)$ denote the proper transforms of the appropriate node scrolls $\left(\mathbb{P}^{1}\right.$-bundles over $\theta$ ) from $\Gamma_{(m)}, \Gamma_{(m-1)}$, respectively (for simplicity of notation, we will drop the tilde over the $C_{\bullet}^{\bullet}$ used in earlier sections), and $C_{i}^{m}=\sum_{\theta} C_{i}^{m}(\theta), C_{i}^{m-1}=\sum_{\theta} C_{i}^{m-1}(\theta) . \quad C_{i}^{m}$ contains the distinguished sections $Q_{i}^{m}, Q_{i+1}^{m}$. The following result is proved similarly to Proposition 2.5:

LEMMA 3.16. We have

$$
\begin{gathered}
\Gamma^{(m)} \cdot \tilde{\Gamma}_{(m)}=-\left(\begin{array}{c}
m \\
2
\end{array}\right) \omega+\sum_{i=1}^{m-1} \nu_{m, i} C_{i}^{m}+\sum_{i=1}^{m-2} \nu_{m, i}^{-} C_{i}^{m-1} \\
\Gamma^{(m-1)} \cdot \tilde{\Gamma}_{(m)}=-\left(\begin{array}{c}
m-1 \\
2
\end{array}\right) \omega+\sum_{i=1}^{m-2} \nu_{m-1, i} C_{i}^{m-1}+\sum_{i=1}^{m-1} \nu_{m-1, i}^{+} C_{i}^{m}
\end{gathered}
$$


where

$$
\begin{array}{r}
\nu_{m, i}=\frac{i(m-i) m}{2}, 1 \leq i \leq m-1, \\
\nu_{m, i}^{-}=\frac{i(m-i-1)(m+1)}{2}, 1 \leq i \leq m-2 \\
\nu_{m-1, i}^{+}=\frac{i(m-i)(m-2)}{2}, 1 \leq i \leq m-1 .
\end{array}
$$

Proof. The coefficient of $C_{i}^{m}$ in $\Gamma^{(m)} \cdot \tilde{\Gamma}_{(m)}$ is already computed in Propoosition 2.5 . As for the coefficient of $C_{i}^{m-1}$ in $\Gamma^{(m)} \cdot \tilde{\Gamma}_{(m)}$, it suffices to note that $C_{i}^{m-1}$ contracts to $Q_{i+1}^{m}$ in $\Gamma_{(m)}$, where $\Gamma^{(m)}$ is defined by $x^{\left(\begin{array}{c}m-i \\ 2\end{array}\right)} y^{\left(\begin{array}{c}i+1 \\ 2\end{array}\right)}$, and $x, y$ have respective multiplicities equal to $i,(m-1-i)$ on $C_{i}^{m-1}$, so the coefficient of $C_{i}^{m-1}$ equals

$$
\left(\begin{array}{c}
m-i \\
2
\end{array}\right) i+\left(\begin{array}{c}
i+1 \\
2
\end{array}\right)(m-i-1)=\frac{i(m-i-1)(m+1)}{2} .
$$

The other formula is proved similarly.

Corollary 3.17 .

$$
\left(\Gamma^{(m)}-\Gamma^{(m-1)}\right) \cdot \Gamma_{(m)}=-(m-1) \omega+\sum_{i=1}^{m-1} i(m-1) C_{i}^{m}+\sum_{i=1}^{m-2} i(m-1-i) C_{i}^{m-1} .
$$

Now set

$$
\begin{array}{r}
\nu_{m}=\sum_{i=1}^{m-1} \nu_{m, i}=\frac{m^{2}\left(m^{2}-1\right)}{12} \\
\nu_{m}^{-}:=\sum_{i=1}^{m-2} \nu_{m, i}^{-}=\sum_{i=1}^{m-1} \nu_{m-1, i}^{+}:=\nu_{m-1}^{+} \\
=\frac{m(m-2)\left(m^{2}-1\right)}{12} .
\end{array}
$$

We begin with a couple of simple examples that don't require the full force of the transfer process.

Example 3.18. Consider a family $X / B$ of arbitrary base dimension, with a map $f: X \rightarrow \mathbb{P}^{m}$. We wish to enumerate the locus of $m$-contact $\mathbb{P}^{m-2}$ 's in the family (e.g. cusps $(m=2)$, inflexional tangent lines $(m=3)$ etc.). More precisely, this is the locus of punctual length- $m$ subschemes of fibres whose image under $f$ is contained in some $\mathbb{P}^{m-2}$; when (and only when) the subscheme is supported at a point $x$ smooth on its fibre, the subscheme is determined by $x$ and denoted $m x$. This locus, of expected codimension 2 , is given by a degeneracy locus of a map over $\Gamma_{(m)}$ :

$$
(m+1) \mathcal{O} \rightarrow \Lambda_{m}(L) .
$$

By Porteous, the locus of these is given by

$$
s_{2, m}=\left(c_{1}^{2}-c_{2}\right)\left(\Lambda_{m}(L)\right) \cap\left[\Gamma_{(m)}\right] .
$$


On $\tilde{\Gamma}_{(m)}$, we can write

$$
c\left(\Lambda_{m}(L)\right)=c\left(\Lambda_{m-1}(L)\right)\left(1+[L]+\Gamma^{(m-1)}-\Gamma^{(m)}\right) .
$$

Therefore,

$$
\begin{aligned}
& s_{2, m}-\tau_{m}^{0}\left(s_{2, m-1}\right) \\
= & c_{1}\left(\Lambda_{m-1}(L)\right)\left(L+\Gamma^{(m-1)}-\Gamma^{(m)}\right)+\left(L+\Gamma^{(m-1)}-\Gamma^{(m)}\right)^{2} \\
= & \left(L+\Gamma^{(m-1)}-\Gamma^{(m)}\right)\left(m L-\Gamma^{(m)}\right) \\
= & m L^{2}-(m+1) L \Gamma^{(m)}+m L \tau_{m}\left(\Gamma^{(m-1)}\right)-\Gamma^{(m)} \tau_{m}\left(\Gamma^{(m-1)}\right)+\left(\Gamma^{(m)}\right)^{2} \\
= & m L^{2}+(m+1)\left(\begin{array}{c}
m \\
2
\end{array}\right) L \omega-(m+1) \sum_{i, \theta} \nu_{m, i} C_{i}^{m}(\theta)\left[\theta^{*} L\right]-m\left(\begin{array}{c}
m-1 \\
2
\end{array}\right) L \omega \\
& +m \sum_{i, \theta} \nu_{m-1, i}^{+} C_{i}^{m}(\theta)\left[\theta^{*} L\right]-\left(\begin{array}{c}
m \\
2
\end{array}\right)\left(\begin{array}{c}
m-1 \\
2
\end{array}\right) \omega^{2}-\Gamma^{(m)} \sum_{i, \theta} \nu_{m-1, i}^{+} C_{i}^{m}(\theta) \\
& +\left(\begin{array}{c}
m \\
2
\end{array}\right)^{2} \omega^{2}+\Gamma^{(m)} \sum_{i, \theta} \nu_{m, i} C_{i}^{m}(\theta) .
\end{aligned}
$$

Therefore

$$
\begin{aligned}
& s_{2, m}-\tau_{m}^{0}\left(s_{2, m-1}\right) \\
= & m L^{2}+m(m-1) L \omega+(m-1)\left(\begin{array}{c}
m \\
2
\end{array}\right) \omega^{2} \\
& -\sum_{i, \theta} \frac{3 i(m-i) m}{2} C_{i}^{m}(\theta)\left[\theta^{*} L\right]+\Gamma^{(m)} \sum_{i, \theta} i(m-i) C_{i}^{m}(\theta) .
\end{aligned}
$$

If $\operatorname{dim}(B)=1$, then $s_{2, m}$ can be viewed as a number, and (3.3.8) gives a recursion for it, one which can be easily solved explicitly, as follows. Note that in this case we have $\theta^{*} L=0$ and $\Gamma^{(m)} C_{i}^{m}(\theta)=-1$, so (3.3.8) simplifies to

$$
\begin{aligned}
s_{2, m}-s_{2, m-1} & =m L^{2}+m(m-1) L \omega+(m-1)\left(\begin{array}{c}
m \\
2
\end{array}\right) \omega^{2}-\sigma \sum_{i, \theta} i(m-i) \\
& =m L^{2}+m(m-1) L \omega+(m-1)\left(\begin{array}{c}
m \\
2
\end{array}\right) \omega^{2}-\sigma \frac{m\left(m^{2}-1\right)}{6} .
\end{aligned}
$$

This recursion is easily integrated, yielding the following Plücker-type formula in closed form:

$$
s_{2, m}=\left(\begin{array}{c}
m+1 \\
2
\end{array}\right) L^{2}+\frac{m\left(m^{2}-1\right)}{2} L \omega+\frac{m\left(m^{2}-1\right)(3 m-2)}{24} \omega^{2}-\frac{m\left(m^{2}-1\right)(m+2)}{24} \sigma
$$

e.g.

$$
s_{2,1}=L^{2}, s_{2,2}=2 L^{2}+3 L \omega+\omega^{2}-\sigma, s_{2,3}=6 L^{2}+12 L \omega+7 \omega^{2}-5 \sigma, \ldots
$$

In case $\operatorname{dim}(B)>1,(3.3 .8)$ must be combined with the punctual transfer calculus (Proposition 3.19 below) to yield the recursion. 
Now we take up the punctual transfer proper. To be precise, let $T_{R}^{0, m}(X / B)$ denote the group generated by $R \subset A \cdot(X)$, the twisted node scrolls $C_{i}^{m}(\theta)[\beta], \beta \in R$ on $\Gamma_{(m)}$, and their sections $\left(-\Gamma^{(m)}\right) C_{i}^{m}(\theta)[\beta]$. Then we will define a 'pointwise transfer' map

$$
\tau_{m}^{0}: T_{R}^{m-1,0}(X / B) \rightarrow T_{R}^{m, 0}(X / B)
$$

that fits in the diagram

$$
\begin{array}{ccc}
T_{R}^{m-1,0}(X / B) & \rightarrow & T_{R}^{m, 0}(X / B) \\
\downarrow & & \downarrow \\
A^{\bullet}\left(\Gamma_{(m-1)}\right) & \rightarrow & A_{\bullet}\left(\Gamma_{(m)}\right) .
\end{array}
$$

Recall that we are assuming $R$ contains $\mathbb{Q}$; this is essential here as $\tau_{m}^{0}$ is only defined over $\mathbb{Q}$. Set

$$
\psi_{i}^{m}:=\psi_{x}^{\otimes\left(\begin{array}{c}
m-i+1 \\
2
\end{array}\right)} \otimes \psi_{y}^{\otimes\left(\begin{array}{l}
i \\
2
\end{array}\right)}
$$

These obviously depend on $\theta$ and will be denoted $\psi_{i}^{m}(\theta)$ when necessary.

The following result gives the main rules of the punctual transfer calculus.

Proposition 3.19. Notations as above, we have, for each node $\theta$,

$$
\tau_{m}^{0}\left(C_{i}^{m-1}(\theta)\right)=\frac{m-i}{m-1} C_{i}^{m}(\theta)+\frac{i+1}{m-1} C_{i+1}^{m}(\theta)
$$

$$
\begin{aligned}
\tau_{m}^{0}\left(-\Gamma^{(m-1)} \cdot C_{i}^{m-1}(\theta)\right)= & \frac{m-i-1}{m} Q_{i+1}^{m}(\theta)+\frac{i+1}{m} Q_{i+2}^{m}(\theta) \\
& +\frac{m-i}{m-1} C_{i}^{m}(\theta)\left[\psi_{i}^{m-1}\right]+\frac{i+1}{m-1} C_{i+1}^{m}(\theta)\left[\psi_{i}^{m-1}\right] \\
= & -\Gamma^{(m)} \cdot C_{i+1}^{m}(\theta)-C_{i+1}^{m}(\theta)\left[\frac{m-i-1}{m} \psi_{i+2}^{m}+\frac{i+1}{m} \psi_{i+1}^{m}\right] \\
& +\frac{m-i}{m-1} C_{i}^{m}(\theta)\left[\psi_{i}^{m-1}\right]+\frac{i+1}{m-1} C_{i+1}^{m}(\theta)\left[\psi_{i}^{m-1}\right] .
\end{aligned}
$$

REMARK 3.20. Because $Q_{i}^{m}=\mathbb{P}\left(\psi_{i}^{m}\right)$ and $Q_{i+1}^{m}$ are disjoint sections on the $\mathbb{P}^{1}$-bundle $C_{i}^{m}=\mathbb{P}\left(\psi_{i}^{m} \oplus \psi_{i+1}^{m}\right)$, we have

$$
\begin{aligned}
Q_{i+1}^{m} \sim Q_{i}^{m}+C_{i}^{m}\left[\psi_{i+1}^{m}\right. & \left.-\psi_{i}^{m}\right]=Q_{i}^{m}+C_{i}^{m}\left[-(m-i) \psi_{x}+i \psi_{y}\right], \\
-\Gamma^{(m)} . C_{i}^{m} & \sim Q_{i}^{m}+C_{i}^{m}\left[\psi_{i+1}^{m}\right] \\
& \sim Q_{i+1}^{m}+C_{i}^{m}\left[\psi_{i}^{m}\right] .
\end{aligned}
$$

REMARK 3.21. If the base $B$ is 1 -dimensional, both $-\Gamma^{(m-1)} C_{i}^{m-1}(\theta)$ and $-\Gamma^{(m)} C_{i}^{m}(\theta)$ are points over the finite set $B(\theta)$ and in particular

$$
\tau_{m}^{0}\left(-\Gamma^{(m-1)} C_{i}^{m-1}(\theta)\right)=-\Gamma^{(m)} C_{i}^{m}(\theta) .
$$


Proof. Fixing (and suppressing) $\theta$, we analyze $\Gamma_{(m)}$ locally over $B$ and near the point $m \theta$ in the small diagonal $X \subset X_{B}^{(m)}$, as in $\S 2.1$. As we have seen, $\Gamma_{(m)}$ is given by the blowup of

$$
J_{m}=\left(g_{i}:=x^{\left(\begin{array}{c}
m-i+1 \\
2
\end{array}\right)} y^{\left(\begin{array}{c}
i \\
2
\end{array}\right)}: i=1, \ldots, m\right)=\prod_{i=1}^{m-1}\left(x^{m-i}, y^{i}\right)
$$

and embeds in

$$
X \times \prod_{i=1}^{m-1} \mathbb{P}_{\left(u_{i}, v_{i}\right)}^{1}
$$

where the $i$ th factor can be identified with $C_{i}^{m}$. The image map $\Gamma_{(m)} \rightarrow X \times C_{i}^{m}$ can be identified with the blowup $X_{i}$ of $X$ in the ideal

$$
\left(g_{i}, g_{i+1}\right)=x^{\left(\begin{array}{c}
m-i \\
2
\end{array}\right)} y^{\left(\begin{array}{c}
i \\
2
\end{array}\right)}\left(x^{m-i}, y^{i}\right)
$$

or what is the same, the blowup of $\left(x^{m-i}, y^{i}\right)$. The pullback of the exceptional divisor in the blowup of $\left(x^{m-i}, y^{i}\right)$, locally defined by $x^{m-i}$ or $y^{i}$, yields a structure of Cartier divisor of multiplicity (=generic length) $i(m-i)$ on $C_{i}^{m}(\theta) \subset \Gamma_{(m)}$. Along $C_{i}^{m}, \Gamma_{(m)}$ is defined by the equation

$$
x^{m-i} u_{i}=y^{i} v_{i}
$$

Also, $C_{i}^{m}$ is endowed with the special sections $Q_{i}^{m}=\left(x^{m-i+1}, y_{i}\right)$, corresponding to $u_{i}=0$, and $Q_{i+1}^{m}=\left(x^{m-i}, y^{i+1}\right)$, corresponding to $v_{i}=0$.

Globally over $B, C_{i}^{m}$ is a $\mathbb{P}^{1}$ bundle of the form

$$
C_{i}^{m}=\mathbb{P}\left(\psi_{i}^{m} \oplus \psi_{i+1}^{m}\right)
$$

(using multiplicative notation for line bundles) with $Q_{i}^{m}=\mathbb{P}\left(\psi_{i}^{m}\right), Q_{i+1}^{m}=\mathbb{P}\left(\psi_{i+1}^{m}\right)$ and $-\Gamma^{(m)}$ corresponding to $\mathcal{O}(1)$. This implies

$$
\mathcal{O}(1) \cdot Q_{j}^{m}=-\Gamma^{(m)} \cdot Q_{j}^{m}=\psi_{j}^{m}, j=i, i+1
$$

Now on $\tilde{\Gamma}_{(m)} \subset X \times \prod_{i=1}^{m-1} C_{i}^{m} \times \prod_{i=1}^{m-2} C_{i}^{m-1}$, the exceptional locus is a connected chain of the form

$$
\tilde{C}_{1}^{m} \cup \tilde{C}_{1}^{m-1} \cup \ldots \cup \tilde{C}_{i}^{m} \cup \tilde{C}_{i}^{m-1} \cup \tilde{C}_{i+1}^{m} \cup \ldots \cup \tilde{C}_{m-1}^{m},
$$

with each $\tilde{C}_{i}^{n}$ projecting isomorphically to $C_{i}^{n}$, and where the intersection $\tilde{C}_{i}^{m} \cap$ $\tilde{C}_{i}^{m-1}$ (resp. $\left.\quad \tilde{C}_{i}^{m-1} \cap \tilde{C}_{i+1}^{m}\right)$ is set-theoretically the section $\left(Q_{i}^{m-1}, Q_{i+1}^{m}\right)$ (resp. $\left(Q_{i+1}^{m-1}, Q_{i+1}^{m}\right)$ ) (the multiplicities will be determined below). From this it follows already that $\tau_{m}^{0}\left(C_{i-1}^{m-1}\right)=a(i-1) C_{i-1}^{m}+b(i-1) C_{i}^{m}$, and it remains to identify $a$ and $b$. This is a consequence of the following Lemma, which completes the proof of $(3.3 .12)$.

LEMMA 3.22. We have:

$$
p_{[m-1]}^{*}\left((m-1) C_{i}^{m-1}\right)=(m-i) \tilde{C}_{i}^{m}+(m-1) \tilde{C}_{i}^{m-1}+(i+1) \tilde{C}_{i+1}^{m},
$$




$$
p_{[m]}^{*}\left(m C_{i}^{m}\right)=(i-1) \tilde{C}_{i-1}^{m-1}+m \tilde{C}_{i}^{m}+(m-1-i) \tilde{C}_{i}^{m-1} .
$$

Proof of Lemma. We will prove (3.3.17) as the case of (3.3.18) is similar. We will analyze this near the flag $\left(Q_{i}^{m-1} \subset Q_{i+1}^{m}\right)$, which is the intersection $\tilde{C}_{i}^{m} \cap \tilde{C}_{i}^{m-1}, i \leq$ $m-2$. There, using homogeneous coordinates $\left[u_{i}^{\prime}, v_{i}^{\prime}\right]$ on $C_{i}^{m-1}=\mathbb{P}^{1}, \tilde{\Gamma}_{(m)}$ has the local equations

$$
\begin{array}{r}
x^{m-i} u_{i}=y^{i} v_{i}, \\
x^{m-1-i} u_{i}^{\prime}=y^{i} v_{i}^{\prime}
\end{array}
$$

which imply

$$
\left(v_{i} / u_{i}\right)\left(u_{i}^{\prime} / v_{i}^{\prime}\right)=x
$$

This means that $x$ cuts out a divisor locally equal to the union of the zero sets of $v_{i}$ ( i.e. $C_{i}^{m-1}$ ), and of $u_{i}^{\prime}$ (i.e. $C_{i}^{m}$ ). Moreover the multiplicity, i.e. local length, of $v_{i}$, along $C_{i}^{m-1}$ is equal to that of $x$, which is the length of $\mathbb{C}[x, y] /\left(x^{m-i}-y^{i}, x\right)$, i.e. $i$. Likewise, the multiplicity of $u_{i}^{\prime}$ along $C_{i}^{m}$ is also equal to $i$. As we have seen in the proof of Proposition 2.5, $u_{i}^{\prime}$, i.e. $u_{i}^{\prime} / v_{i}^{\prime}$, is a local defining equation on $\Gamma_{(m-1)}$ for the Cartier divisor $(m-1) C_{i-1}^{m-1}$. It follows, under the assumption $i \leq m-2$, that in the pullback of $(m-1) C_{i-1}^{m-1}$ to $\tilde{\Gamma}_{(m)}, C_{i}^{m}$ appears with multiplicity $i$. In the extreme case of $C_{m-2}^{m-1}$, both it and $C_{m-1}^{m}$ clearly have multiplicity 1 on $\tilde{\Gamma}_{(m)}$, so $C_{m-1}^{m}$ appears with multiplicity $m-1$ in the pullback of $(m-1) C_{m-2}^{m-1}$.

Similarly using the relation

$$
\left(u_{i+1} / v_{i+1}\right)\left(v_{i}^{\prime} / u_{i}^{\prime}\right)=y
$$

the pullback of $(m-1) C_{i+1}^{m-1}$, contains $C_{i+1}^{m}$ with multiplicity $m-i-1$ for all $i<m-2$.

Thus proves (3.3.17) by shifting the index $i$.

For (3.3.18) briefly, the same relations (3.3.20) and (3.3.21) show that the respective multiplicities of the pullback of $m C_{i+1}^{m}$ (resp. $m C_{i}^{m}$ ) along $C_{i}^{m-1}$ are equal to $i$ (resp. $m-1-i$ ).

To obtain (3.3.13), we continue to develop intersection theory on the punctual flaglet Hilbert scheme, using the same notations.

LEMMA 3.23. We have

$$
\begin{gathered}
\tilde{C}_{i}^{m-1} \tilde{C}_{i}^{m}=\frac{1}{i}\left(Q_{i}^{m-1}, Q_{i+1}^{m}\right), \\
\tilde{C}_{i}^{m-1} \tilde{C}_{i+1}^{m}=\frac{1}{m-i-1}\left(Q_{i+1}^{m-1}, Q_{i+1}^{m}\right), i<m-1 .
\end{gathered}
$$

Proof of Lemma. For the first relation, the identity (3.3.20) shows that the Cartier divisors $i \tilde{C}_{i}^{m-1}, i \tilde{C}_{i}^{m}$ meet with multiplicity $i$ along $\left(Q_{i}^{m-1}, Q_{i}^{m}\right)$. For the second relation, use similarly the identity (3.3.21).

LEMMA 3.24. We have:

$$
\left(\tilde{C}_{i}^{m}\right)^{2}=-\frac{m-1}{m}\left(\frac{1}{m-i}\left(Q_{i}^{m-1}, Q_{i}^{m}\right)+\frac{1}{i}\left(Q_{i}^{m-1}, Q_{i+1}^{m}\right)\right) .
$$


Proof of Lemma. The projection formula, together with Lemma 3.22 shows that

$$
p_{[m] *}\left(\left(\tilde{C}_{i}^{m}\right)^{2}\right)=\left(C_{i}^{m}\right)^{2}-p_{[m] *}\left(\tilde{C}_{i}^{m} \cdot\left((i-1) \tilde{C}_{i-1}^{m-1}+(m-1-i) \tilde{C}_{i}^{m-1}\right)\right) .
$$

Combining this with Corollary 2.6, (ii) and Lemma 3.23, we get the result by simple arithmetic.

Similarly, we can show:

LEMMA 3.25.

$$
\left(\tilde{C}_{i}^{m-1}\right)^{2}=-\frac{m}{m-1}\left(\frac{1}{i}\left(Q_{i}^{m-1}, Q_{i+1}^{m}\right)+\frac{1}{m-i-1}\left(Q_{i+1}^{m-1}, Q_{i+1}^{m}\right)\right) .
$$

Now we are in position to compute the punctual transfer of a section. We have

$$
-\Gamma^{(m-1)} \cdot C_{i}^{m-1}=Q_{i+1}^{m-1}+C_{i}^{m-1}\left[\psi_{i}^{m-1}\right]=(m-1) C_{i}^{m-1} \cdot C_{i+1}^{m-1}+C_{i}^{m-1}\left[\psi_{i}^{m-1}\right] .
$$

The transfer of the second summand above is computed directly by (3.3.12) and yields the last two terms in the first equality in (3.3.13). As for the first summand above, its pullback on $\tilde{\Gamma}_{(m)}$ is given by

$$
(m-1)\left(\tilde{C}_{i}^{m-1}+\frac{m-i}{m-1} \tilde{C}_{i}^{m}+\frac{i+1}{m-1} \tilde{C}_{i+1}^{m}\right)\left(\tilde{C}_{i+1}^{m-1}+\frac{m-i-1}{m-1} \tilde{C}_{i+1}^{m}+\frac{i+2}{m-1} \tilde{C}_{i+2}^{m}\right) .
$$

The only terms not clearly trivial come from $\tilde{C}_{i}^{m-1} \cdot \tilde{C}_{i+1}^{m}, \tilde{C}_{i+1}^{m-1} \cdot \tilde{C}_{i+1}^{m}$ and $\left(\tilde{C}_{i+1}^{m}\right)^{2}$, and those be computed using the above Lemmas, yielding

$$
\frac{m-i-1}{m}\left(Q_{i+1}^{m-1}, Q_{i+1}^{m}\right)+\frac{i+1}{m}\left(Q_{i+1}^{m-1}, Q_{i+2}^{m}\right) .
$$

By projection, we obtain the first equality in (3.3.13). The second equality is immediate from Remark 3.20.

This completes the proof of Proposition 3.19.

REMARK 3.26. Using (3.3.26) and Remark 3.20 again, one can formulate the punctual transfer entirely in terms of node scrolls $C_{i}^{m}$ and the canonical sections $Q_{i}^{m}$, via

$$
\begin{aligned}
\tau_{m}^{0}\left(Q_{i}^{m-1}\right) & =Q_{i}^{m}+C_{i}^{m}\left[\frac{-i(m-i)}{m} \psi_{x}+\frac{i^{2}}{m} \psi_{y}\right] \\
& =\frac{m-i}{m} Q_{i}^{m}+\frac{i}{m} Q_{i+1}^{m} .
\end{aligned}
$$

Remark 3.27. By simple numerology, Proposition 3.19 can be used to reprove Lemma 3.16. We omit the details.

Proposition 3.19 leads, in a completely straightforward way, to a recursive formula for the total Chern class of the tautological bundles $\left.\Lambda_{m}(L)\right|_{\Gamma_{(m)}}$ for a line bundles $L$. Write recursively

$$
\begin{aligned}
c_{m}:=c\left(\left.\Lambda_{m}(L)\right|_{\Gamma_{(m)}}\right) & =\alpha_{m}+\sum_{\theta}\left(\sum_{i=1}^{m-1} C_{i}^{m}(\theta)\left[\beta_{m}^{i}(\theta)\right]+\sum_{i=1}^{m-1} \gamma_{m}^{i}(\theta) Q_{i}^{m}(\theta)\right), \\
\alpha & \in R, \beta(\theta), \gamma(\theta) \in A \cdot(B(\theta)), \alpha_{1}=1+L, \beta_{i}=\gamma_{i}=0 .
\end{aligned}
$$


where $B(\theta) \subset B$ is the normalization of the boundary divisor corresponding to $\theta$ and a cycle on $B(\theta)$ is viewed as a cycle on $B$ via the Gysin map. In view of Remark 3.20, such expressions are not unique, but this does not matter. In the ensuing computation we will suppress the $\theta$ summation, which will be understood.

We have:

$$
c_{m}=\tau_{m}^{0}\left(c_{m-1}\left(1+L+\Gamma^{(m-1)}\right)\right)+\left(-\Gamma^{(m)}\right) \tau_{m}^{0}\left(c_{m-1}\right) .
$$

The first summand yields

$$
\begin{aligned}
& \left(1+L-\left(\begin{array}{c}
m-1 \\
2
\end{array}\right) \omega+\frac{1}{m-1} \sum_{i=1}^{m-2} \nu_{m-1, i}\left((m-i) C_{i}^{m}+(i+1) C_{i+1}^{m}\right)\right) \alpha_{m-1} \\
& +\frac{1}{m-1} \sum C_{i}^{m}\left[\beta_{m-1}^{i}(m-i)(1+L)\right]+C_{i+1}^{m}\left[\beta_{m-1}^{i}(i+1)(1+L)\right] \\
& -\frac{1}{m-1} \sum \beta_{m-1}^{i} \psi_{i+1}^{m-1}\left((m-i) C_{i}^{m}+(i+1) C_{i+1}^{m}\right. \\
& +\frac{1}{m} \sum_{i=1}^{m-1}\left((1+L) \gamma_{m-1}^{i}-\beta_{m-1}^{i}\right)\left((m-i) Q_{i}^{m}+i Q_{i+1}^{m}\right)
\end{aligned}
$$

(where $\theta^{*}$ means pullback by the node-section $\theta: B(\theta) \rightarrow X$ and products involving classes on $B(\theta)$ are performed on $B(\theta))$.

Thus in all

$$
\begin{aligned}
\alpha_{m}= & (1+L+(m-1) \omega) \alpha_{m-1}=\prod_{i=1}^{m}(1+L+(i-1) \omega) \\
\beta_{m}^{i}= & \frac{1}{m-1}\left(\left((m-i)\left(1+L-\psi_{i+1}^{m-1}\right)+\psi_{i+1}^{m}\right) \beta_{m-1}^{i}\right. \\
& \left.+\left(i\left(1+L-\psi_{i}^{m-1}\right)+\psi_{i+1}^{m}\right) \beta_{m-1}^{i-1}\right)-\nu_{m, i} \alpha_{m-1} ; \\
\gamma_{m}^{i}= & \left.\frac{1}{m}\left((m-i)\left(1+L+\psi_{i}^{m}\right) \gamma_{m-1}^{i}\right)+(i-1)\left(1+L+\psi_{i}^{m}\right) \gamma_{m-1}^{i-1}\right) \\
& +\frac{1}{m(m-1)}\left((m-i) \beta_{m-1}^{i}+(m+i-1) \beta_{m-1}^{i-1}\right) .
\end{aligned}
$$

We have used the fact that $\theta^{*}\left(\alpha_{m-1}\right)=\theta^{*}\left((1+L)^{m-1}\right)$, which follows from the above formula for the $\alpha_{m}$, plus the fact that $\theta^{*}(\omega)=0$. We have proven

COROLlary 3.28. The Chern classes of the tautological bundle on the punctual Hilbert scheme $\Gamma_{(m)}(X / B)$ are given by (3.3.28), where the coefficients satisfy the recursion (3.3.29).

EXAmple 3.29. Given a family $X / B$ (of any base dimension), and a map

$$
f: X \rightarrow \mathbb{P}^{n}, n<m
$$

$\left.c_{m-n}\left(\left.\Lambda_{m}\left(f^{*}(\mathcal{O}(1))\right)\right|_{\Gamma_{(m)}}\right]\right)$ represents the locus, finite if $\operatorname{dim}(B)=m-n-1$, of points in $X$ where the fibre admits an $m$-contact hyperplane. If $n=1$, this is the locus of $(m-1)$ st order ramification points. If $n=2$, it is the locus of $m$-th order hyperflexes, etc. 
The case $n=m-2$ can be worked out more explicitly, as in Example 3.18. Let $\left.L=f^{*}(\mathcal{O}(1))\right), c_{2, m}=c_{2}\left(\left.\Lambda_{m}(L)\right|_{\Gamma_{(m)}}\right)$.

$$
\begin{aligned}
c_{2, m}-\tau_{m}^{0}\left(c_{2, m-1}\right) & =\left((m-1) L-\Gamma^{(m-1)}\right)\left(L-\Gamma^{(m)}+\Gamma^{(m-1)}\right) \\
& =(m-1) L^{2}+(m-1)\left(\begin{array}{c}
m-1 \\
2
\end{array}\right) \omega^{2}+\left(\left(\begin{array}{c}
m-1 \\
2
\end{array}\right)+(m-1)^{2}\right) L \omega \\
& -\sum \nu_{m-1, i}^{+} C_{i}^{m}\left[\theta^{*} L\right]+(m-1) \sum\left(\nu_{m-1, i}^{+}-\nu_{m, i}\right) C_{i}^{m}\left[\theta^{*} L\right] \\
& +\Gamma^{(m)} \sum \nu_{m-1, i}^{+} C_{i}^{m}-\sum \nu_{m-1, i} \tau_{m}\left(\Gamma^{(m-1)} C_{i}^{m-1}\right) .
\end{aligned}
$$

Then simple computations and Remark 3.21 yield in the pencil case

$$
\begin{aligned}
& c_{2, m}-\tau_{m}^{0}\left(c_{2, m-1}\right)= \\
& (m-1) L^{2}+(m-1)\left(\begin{array}{c}
m-1 \\
2
\end{array}\right) \omega^{2}+\frac{(m-1)(3 m-4)}{2} L \omega \\
& -\sum_{\theta} \sum_{i=1}^{m-1} \frac{i(m-i)}{2}\left((3 m-4) C_{i}^{m}(\theta)\left[\theta^{*} L\right]\right)-\left(\begin{array}{c}
m \\
3
\end{array}\right) \sigma .
\end{aligned}
$$

Again in the pencil case $\operatorname{dim}(B)=1$ these classes can be viewed as numbers, $\theta^{*} L$ and $\psi_{i}^{m-1}$ are all zero and the above simplifies to

$$
\begin{aligned}
& c_{2, m}-c_{2, m-1}= \\
& (m-1) L^{2}+(m-1)\left(\begin{array}{c}
m-1 \\
2
\end{array}\right) \omega^{2}+\frac{(m-1)(3 m-4)}{2} L \omega-\left(\begin{array}{c}
m \\
3
\end{array}\right) \sigma .
\end{aligned}
$$

This recursion can be integrated easily using the elementary formula $\sum_{n=k}^{m}\left(\begin{array}{c}n \\ k\end{array}\right)=\left(\begin{array}{c}m+1 \\ k+1\end{array}\right)$, yielding the following closed-form Plücker-type formula:

$$
c_{2, m}=\left(\begin{array}{c}
m \\
2
\end{array}\right) L^{2}+\left(3\left(\begin{array}{c}
m+1 \\
4
\end{array}\right)-\left(\begin{array}{c}
m \\
3
\end{array}\right)\right) \omega^{2}+\left(3\left(\begin{array}{c}
m+1 \\
3
\end{array}\right)-2\left(\begin{array}{c}
m \\
2
\end{array}\right)\right) L \omega-\left(\begin{array}{c}
m+1 \\
4
\end{array}\right) \sigma .
$$

Added in proof: Francesco Cavazzani (student of Joe Harris at Harvard) communicated in Dec 2012 that he had checked (3.3.31) by another method.

\section{Low-degree examples.}

4.1. Trisecants to one space curve curve. If $X$ is a smooth curve of degree $d$ and genus $g$ in $\mathbb{P}^{3}$, the virtual degree of its trisecant scroll, i.e. the virtual number of trisecant lines to $X$ meeting a generic line, is given by $c_{3}\left(\bigwedge^{2}\left(\Lambda_{3}\left(\mathcal{O}_{X}(1)\right)\right)\right.$, which can be easily computed to be

$$
\frac{1}{6}\left(2 d^{3}-12 d^{2}+16 d-3 d(2 g-2)+6(2 g-2)\right) .
$$

4.2. Multisecants in a pencil . Let $X / B$ be a family of nodal curves over a smooth curve, and suppose

$$
f: X \rightarrow \mathbb{P}^{2 m-1}
$$


is a morphism. One, quite special, class of examples of this situation arises as what we call a generic rational pencil; that is, generally, the normalization of the family of rational curves of fixed degree $d$ in $\mathbb{P}^{r}$ (so $r=2 m-1$ here) that are incident to a generic collection $A_{1}, \ldots A_{k}$ of linear spaces, with

$$
(r+1) d+r-4=\sum\left(\operatorname{codim}\left(A_{i}\right)-1\right)
$$

see [20] and references therein, or [18]. Our result applies to curves of arbitrary genus.

Returning to the general situation, one expects a finite number $N_{m}$ of curves $f\left(X_{b}\right)$ to admit an m-secant $(m-2)$-plane. Let $L=f^{*} \mathcal{O}(1), V=$ $H^{0}\left(\mathbb{P}^{2 m-1}\right), c(m, i)=c_{i}\left(\Lambda_{m}(L)\right)$. Then $N_{m}$ is the degree of the locus where the natural map $V \otimes \mathcal{O}_{X_{B}^{[m]}} \rightarrow \Lambda_{m}(L)$ drops rank. By Porteous' formula [6], this number can be evaluated as

$$
\int_{X_{B}^{[m]}} \Delta_{1}^{(m+1)}(c(m, .)) .
$$

For convenience, we will denote $m$ ! times this number simply by $\Delta_{1}^{(m+1)}$. These numbers have been evaluated by completely different means (namely, 'test pencils' as in the Harris-Mumford paper) by Ethan Cotteril [5], [4], [3]. For $m=3$, our intersection theory yields the same number after prolonged manual calculations:

$$
1 / 6\left(\left(3 d^{2}-27 d+60\right) L^{2}+(-12 d+72) L . \omega+(-3 d+28) \omega^{2}-3 b(2 g-2)+(3 d-20) \sigma\right)
$$

where $d$ is the degree of a fibre in $\mathbb{P}^{2 m-1}$ and $\sigma$ is the number of singular fibres.

More generally, for any 1-parameter family as above, natural numbers $r, s$ with $r s=m+1$ and a map

$$
f: X \rightarrow \mathbb{P}^{(r+1)(s-1)-1}
$$

we have a 'Porteous number'

$$
\Delta_{r}^{(s)}=m ! \int_{X_{B}^{[m]}} \Delta_{r}^{(s)}(c(m, .))=\int_{W^{m}(X / B)} \Delta_{r}^{(s)}(c(m, .))
$$

which counts $m$ ! times the virtual number of $m$-secant $\mathbb{P}^{m-r-1}$-planes in the family.

Beyond $m=3$, manual calculation via our intersection calculus seems impractical; fortunately, we have macnodal, discussed next.

4.3. Gwoho Liu's Macnodal program. Gwoho Liu has written a Java program [12] which implements the above intersection theory, both on a fixed Hilbert scheme $X_{B}^{[m]}$ and, together with the transfer calculus of $\S 3$, on a flag scheme $W^{m}(X / B)$. This is sufficient to evaluate all Chern numbers of tautologocal bundles and in particular all the aforementiond multisecant numbers. We proceed with some examples of Macnodal computations. Details about Macnodal, and further examples, will be given elsewhere.

EXAmple 4.1 (pencils). As above, let $c(m, i)=c_{i}\left(\Lambda_{m}(L)\right), L=f^{*}(\mathcal{O}(1), f$ : $X \rightarrow \mathbb{P}^{2 m-1}$, for a good pencil $X / B$. When $m=3, r=1$, Macnodal yields the 
formula above. When $m=4, r=1$, it computes the number of $m$-secant $(m-2)$ planes in the family (times $m$ !) as

$$
\begin{aligned}
\Delta_{1}^{(5)}= & c(4,1)^{5}-4 c(4,2) c(4,1)^{3}+3 c(4,3) c(4,1)^{2}+3 c(4,2)^{2} c(4,1) \\
& -2 c(4,1) c(4,4)-2 c(4,3) c(4,2) \\
= & \left(-1008+452 d-72 d^{2}+4 d^{3}-24 d g+168 g\right) L^{2}+\left(432-98 d+6 d^{2}-12 g\right) \sigma \\
& +\left(-1440+360 d-24 d^{2}+48 g\right) \omega \cdot L+\left(-720+130 d-6 d^{2}+12 g\right) \omega^{2} .
\end{aligned}
$$

Similarly, we have

$$
\begin{aligned}
c(5,1)^{6}= & \left(1483200-1022400 d+280800 d^{2}-10800 d^{2} g-36000 d^{3}\right. \\
& \left.+1800 d^{4}+122400 d g-363600 g+5400 g^{2}\right) L . L \\
& +\left(-400800+162000 d-23400 d^{2}+1200 d^{3}-3600 d g+25800 g\right) \sigma \\
& +\left(1843200-820800 d+129600 d^{2}-7200 d^{3}+21600 d g-144000 g\right) w . L \\
& +\left(775200-262800 d+30600 d^{2}-1200 d^{3}+3600 d g-33000 g\right) w . w .
\end{aligned}
$$

This is one of 10 terms in $\Delta_{1}^{(6)}\left(5\right.$-secant planes of a pencil in $\left.\mathbb{P}^{9}\right)$ :

$$
\begin{aligned}
\Delta_{1}^{(6)}= & \left(19560-9270 d+1735 d^{2}-60 d^{2} g-150 d^{3}+5 d^{4}+1020 d g-4500 g+60 g^{2}\right) L . L \\
& +\left(-10720+2960 d-290 d^{2}+10 d^{3}-60 d g+640 g\right) \sigma \\
& +\left(33600-10160 d+1080 d^{2}-40 d^{3}+240 d g-2400 g\right) w . L \\
& +\left(20000-4640 d+370 d^{2}-10 d^{3}+60 d g-800 g\right) w . w .
\end{aligned}
$$

For 5 -secant planes of a pencil in $\mathbb{P}^{5}$ :

$$
\begin{aligned}
\Delta_{2}^{(3)}= & \left(17400-11070 d+2805 d^{2}-120 d^{2} g-330 d^{3}\right. \\
& \left.+15 d^{4}+1500 d g-4860 g+60 g^{2}\right) L \cdot L \\
& +\left(-4640+1630 d-210 d^{2}+10 d^{3}-60 d g+440 g\right) \sigma \\
& +\left(21600-8520 d+1200 d^{2}-60 d^{3}+300 d g-2160 g\right) w . L \\
& +\left(9280-2670 d+270 d^{2}-10 d^{3}+60 d g-520 g\right) w . w .
\end{aligned}
$$

5-secant lines for a pencil in $\mathbb{P}^{3}$ :

$(4.3 .5)$

$$
\begin{aligned}
\Delta_{3}^{2}= & \left(5160-4020 d+1250 d^{2}-60 d^{2} g-180 d^{3}+10 d^{4}+600 d g-1620 g+60 g^{2}\right) L \cdot L \\
& +\left(-640+210 d-20 d^{2}+40 g\right) \sigma \\
& +\left(4800-2200 d+360 d^{2}-20 d^{3}+60 d g-480 g\right) w \cdot L \\
& +\left(1520-450 d+40 d^{2}-80 g\right) w \cdot w .
\end{aligned}
$$


7-secant $\mathbb{P}^{4}$-s for a pencil in $\mathbb{P}^{9}$ :

(4.3.6)

$$
\begin{aligned}
\Delta_{2}^{(4)}= & \left(23420880-15283296 d+4337172 d^{2}-592200 d^{2} g+5040 d^{2} g^{2}-679140 d^{3}\right. \\
& +44100 d^{3} g+61425 d^{4}-1260 d^{4} g-3024 d^{5}+63 d^{6} \\
& \left.+3633840 d g-95760 d g^{2}-8646120 g+468720 g^{2}-2520 g^{3}\right) L . L \\
& +\left(-10425240+4489296 d-819630 d^{2}+39060 d^{2} g+78960 d^{3}-1260 d^{3} g\right. \\
& \left.-3990 d^{4}+84 d^{5}-418740 d g+2520 d g^{2}+1563240 g-28560 g^{2}\right) \sigma \\
& +\left(40007520-18793404 d+3700620 d^{2}-187740 d^{2} g-379575 d^{3}+6300 d^{3} g\right. \\
& \left.+20160 d^{4}-441 d^{5}+1922760 d g-12600 d g^{2}-6804000 g+136080 g^{2}\right) w \cdot L \\
& +\left(23373000-8857296 d+1396710 d^{2}-46620 d^{2} g-114240 d^{3}+1260 d^{3} g\right. \\
& \left.+4830 d^{4}-84 d^{5}+586740 d g-2520 d g^{2}-2536800 g+34440 g^{2}\right) w \cdot w .
\end{aligned}
$$

7-secant planes for a pencil in $\mathbb{P}^{4}$ :

(4.3.7)

$$
\begin{aligned}
\Delta_{4}^{(2)}= & \left(2242800-1966440 d+750680 d^{2}-109200 d^{2} g+1260 d^{2} g^{2}-157920 d^{3}\right. \\
& +10920 d^{3} g+19145 d^{4}-420 d^{4} g-1260 d^{5}+35 d^{6}+500640 d g-17640 d g^{2} \\
& \left.-896280 g+65520 g^{2}-840 g^{3}\right) L \cdot L \\
& +\left(-400680+200340 d-40180 d^{2}+840 d^{2} g+3780 d^{3}-140 d^{4}\right. \\
& \left.-12180 d g+48720 g-840 g^{2}\right) \sigma \\
& +\left(2646000-1619100 d+415800 d^{2}-20160 d^{2} g-55335 d^{3}+840 d^{3} g+3780 d^{4}\right. \\
& \left.-105 d^{5}+164220 d g-1260 d g^{2}-466200 g+12600 g^{2}\right) \omega \cdot L \\
& +\left(1040760-483420 d+90440 d^{2}-1680 d^{2} g-7980 d^{3}+280 d^{4}\right. \\
& \left.+25620 d g-110040 g+1680 g^{2}\right) \omega^{2} .
\end{aligned}
$$

8-secant $\mathbb{P}^{4}$-s of a pencil in $\mathbb{P}^{7}$ :

(4.3.8)

$$
\begin{aligned}
\Delta_{3}^{(3)}= & -524966400+399120960 d-135158352 d^{2}+25242000 d^{2} g-564480 d^{2} g^{2} \\
& +26227992 d^{3}-2807280 d^{3} g+20160 d^{3} g^{2}-3129840 d^{4}+159600 d^{4} g+228480 d^{5} \\
& -3696 d^{5} g-9408 d^{6}+168 d^{7}-116556384 d g+5382720 d g^{2}-20160 d g^{3}+222243840 g \\
& \left.-17539200 g^{2}+201600 g^{3}\right) L . L \\
& +\left(193334400-98974848 d+22152760 d^{2}-1627080 d^{2} g+10080 d^{2} g^{2}-2761220 d^{3}\right. \\
& +102480 d^{3} g+201180 d^{4}-2520 d^{4} g-8092 d^{5}+140 d^{6}+11973360 d g-215040 d g^{2} \\
& \left.-34487040 g+1186080 g^{2}-3360 g^{3}\right) \sigma \\
& +\left(-853493760+482291712 d-118440672 d^{2}+9760800 d^{2} g-60480 d^{2} g^{2}\right. \\
& +16089360 d^{3}-651840 d^{3} g-1268400 d^{4}+16800 d^{4} g+54768 d^{5}-1008 d^{6} \\
& \left.-67085760 d g+1249920 d g^{2}+179141760 g-6632640 g^{2}+20160 g^{3}\right) \omega \cdot L \\
& +\left(-469929600+216819456 d-43094520 d^{2}+2299080 d^{2} g-10080 d^{2} g^{2}+4692100 d^{3}\right. \\
& -122640 d^{3} g-293020 d^{4}+2520 d^{4} g+9884 d^{5}-140 d^{6}-19634160 d g+248640 d g^{2} \\
& \left.+64431360 g-1575840 g^{2}+3360 g^{3}\right) \omega^{2} .
\end{aligned}
$$


In all the cases where $r=1$ (i.e. computing $\Delta_{r}^{(s)}$ ) or the pencil is in $\mathbb{P}^{r}$, these formulas match those previously obtained by Cotteril [3] by different methods (which don't seem to generalize readily to the case of higher base dimension).

EXAmple 4.2 (beyond pencils). Macnodal is applicable in any base dimension, though in the case $\operatorname{dim}(B)>1$, there are apparently no examples in the literature to compare to. Here is a 2-dimensional example. Let $X / B$ let a 2-parameter family, $E$ a rank- 6 bundle over $B$ with Chern classes $d_{i}$, and $\phi: E \rightarrow \Lambda_{5}(L)$ a map. The locus where $\phi$ has rank at most 3 , is enumerated by

Macnodal> cc(Delta $\left(\right.$ dim_b$\left.\left._{-}=2,2,2,2\right)\right)$

$$
\begin{aligned}
& \left(144-204 d+106 d^{2}-12 d^{2} g-24 d^{3}+2 d^{4}+84 d g-156 g+12 g^{2}\right) \int_{B_{0}} d_{1}^{2} \\
& +\left(1152-708 d+156 d^{2}-12 d^{3}+48 d g-252 g\right) \int_{B_{0}} d_{1} \pi_{*}\left(L^{2}\right) \\
& +\left(1152-384 d+36 d^{2}-60 g\right) \int_{B_{0}} d_{1} \pi_{*}(L \omega)+\left(402-90 d+6 d^{2}-12 g\right) \int_{B_{0}} d_{1} \pi_{*}\left(\omega^{2}\right) \\
& +\left(216-258 d+107 d^{2}-12 d^{2} g-18 d^{3}+d^{4}+108 d g-216 g\right) \int_{B_{0}} d_{2} \\
& +\left(387-132 d+12 d^{2}-12 g\right) \int_{B_{0}} \pi_{*}\left(L^{2}\right)^{2}+(378-60 d) \int_{B_{0}} \pi_{*}\left(L^{2}\right) \pi_{*}(L \omega) \\
& +(92-12 d) \int_{B_{0}} \pi_{*}\left(L^{2}\right) \pi_{*}\left(\omega^{2}\right)+\left(-2778+582 d-30 d^{2}+48 g\right) \int_{B_{0}} \pi_{*}\left(L^{2} \omega\right) \\
& +\left(-1668+624 d-84 d^{2}+4 d^{3}-24 d g+168 g\right) \int_{B_{0}} \pi_{*}\left(L^{3}\right)+51 \int_{B_{0}} \pi_{*}(L \omega)^{2}+24 \int_{B_{0}} \pi_{*}(L \omega) \pi_{*}\left(\omega^{2}\right) \\
& +\left(-1962+266 d-6 d^{2}+12 g\right) \int_{B_{0}} \pi_{*}\left(L \omega^{2}\right)+3 \int_{B_{0}} \pi_{*}\left(\omega^{2}\right)^{2}+(-540+48 d) \int_{B_{0}} \pi_{*}\left(\omega^{3}\right) \\
& +\left(-222+66 d-6 d^{2}+12 g\right) \int_{B_{1}} d_{1}+(-76+12 d) \int_{B_{1}} \pi_{*}\left(L^{2}\right) \quad-24 \int_{B_{1}} \pi_{*}(L \omega)-6 \int_{B_{1}} \pi_{*}\left(\omega^{2}\right) \\
& +(207-24 d) \int_{B_{1}} \psi_{1}^{(1)}+\left(1014-178 d+6 d^{2}-12 g\right) \int_{B_{1}} \theta_{1}^{*}(L)+3 \int_{B_{2}} 1
\end{aligned}
$$

Here $B_{i} \rightarrow B$ is the normalization of the locus of $i$-nodal curves, $\theta_{1} \rightarrow X_{B_{1}}$ is the node of the restricted family, and $\psi_{1}^{(1)}$ is the sum of the cotangent classes at the branches of $X$ at $\theta_{1}$. Also, in integrals over $B_{i}, \omega$ refers to the dualizing sheaf of the normalization of $X_{B_{i}}$.

More examples and details about using Macnodal will be given elsewhere. 
4.4. Double points. Let $X / B$ be an arbitrary nodal family and $f: X \rightarrow \mathbf{P}^{n}$ a morphism. Consider the relative double points of $f$, i.e. double points on fibres. This locus on $X_{B}^{[2]}$ is the degeneracy locus of a bundle map

$$
\phi:(n+1) \mathcal{O} \rightarrow \Lambda_{2}(L), L:=f^{*} \mathcal{O}(1) .
$$

By Porteous, the virtual fundamental class of this locus is given by the Segre class $s_{n}\left(\Lambda_{2}(L)^{*}\right)$, whose pullback on the ordered (= flag) Hilbert scheme $X_{B}^{\lceil 2\rceil}$ equals

$$
\sum_{i=0}^{n}\left(L_{1}\right)^{n-i}\left(L_{2}-\Gamma\right)^{i}, \Gamma=\Gamma^{\lceil 2\rceil} .
$$

The powers of $\Gamma$ can be evaluated using Corollary 2.30. Pushing the result down to $X_{B}^{2}$ for simplicity yields

$$
\sum_{i=0}^{n} L_{1}^{n-i} L_{2}^{i}-\sum_{i=0}^{n} L_{1}^{n-i}\left(\sum_{j=1}^{i}\left(\Gamma\left[\omega^{j-1}\right]+\sum_{s, k} \delta_{s *}\left(\psi_{x}^{j-2-k} \psi_{y}^{k}\right)\right) L^{i-j}\right) .
$$

To describe the direct image of this on $B$, we need some notation. Recall that $\kappa_{j}=$ $\pi_{*}\left(\omega^{j+1}\right)$. Extending this, we may set

$$
\kappa_{j}(L)=\pi_{*}\left(L^{j+1}\right), \kappa_{i, j}(L, M)=\pi_{*}\left(L^{i+1} M^{j+1}\right) .
$$

Note that in our case $\kappa_{j}(L)$ may be interpreted as the class of the locus of curves meeting a generic $\mathbb{P}^{n-j}$. Also, for each boundary datum $\left(T_{s}, \delta_{s}, \theta_{s}\right), T_{s}$ admits a map to $\mathbb{P}^{n}$ via either the $x$ or $y$-section (the two maps are the same), via which we can pull back $L^{j}$, which corresponds to the locus of boundary curves whose node $\theta_{s}$ meets $\mathbb{P}^{n-j}$. Then pushing the above down to $B$ yields the following

Proposition 4.3. Notations as above, the virtual class of the locus of fibres not embedded by $f$ is

$$
m_{2, B}=\frac{1}{2}(-1)^{n}\left(\sum_{i=1}^{n-1} \kappa_{i-1}(L) \kappa_{n-i-1}(L)-\kappa_{n-j-1, j-2}(L, \omega)+\sum_{s, k} \delta_{s *}\left(L^{n-j} \psi_{x}^{j-2-k} \psi_{y}^{k}\right)\right) .
$$

More generally, for any smooth variety $Y$ of dimension $n$ and map $f: X \rightarrow Y$, one can use the double-point formula of [22], Th. 3.3ter, p. 1208, to evaluate the class of the double-point locus in $X_{B}^{2}$ in terms of the diagonal class $\Delta_{Y}$ on $Y \times Y$ as

$$
\begin{aligned}
2 m(f)_{X_{B}^{2}} & =\left(f^{2}\right)^{*}\left(\Delta_{Y}\right)-\sum_{i \geq 1}\left(-\Gamma^{i}\right) c_{n-i}\left(T_{Y}\right) \\
& =\left(f^{2}\right)^{*}\left(\Delta_{Y}\right)-\sum_{i \geq 1}\left(-\Gamma\left[\omega^{i-1}\right]+\frac{1}{2} \sum_{s, j} \delta_{s *}\left(\psi_{x}^{i-j-3} \psi_{y}^{j}\right)\right) c_{n-i}\left(T_{Y}\right)
\end{aligned}
$$

(here each boundary term corresponding to a node $\theta$ is embedded in the diagonal in $X_{B}^{2}$ via $\theta$ ). Applying this set-up to the case $L=\omega, Y=\mathbb{P}(\mathbb{E}), \mathbb{E}$ being the Hodge bundle, and replacing $T_{Y}$ and $\Delta_{Y}$ be their relative analogues over $B$, we note that

$$
\begin{aligned}
& {\left[\Delta_{Y / B}\right]=\left\{\frac{c\left(\mathbb{E}^{*}\right)}{\left(1-\left[L_{1}\right]\right)\left(1-\left[L_{2}\right]\right)}\right\}_{g-1}, L_{i}=p_{i}^{*} \mathcal{O}(1),} \\
& c_{i}\left(T_{Y / B}\right)=\sum(-1)^{k}\left(\begin{array}{c}
g-k \\
i-k
\end{array}\right) \lambda_{k} L^{i-k}, \lambda_{k}=c_{k}(\mathbb{E}) .
\end{aligned}
$$


Applying this to (4.4.3), we note that $L$ pulls back to $\omega$, which meets each $\theta$ trivially, so we obtain

$$
\begin{aligned}
2 m_{X_{B}^{2}}(f)= & \sum(-1)^{k} \lambda_{k}\left[\omega^{i}, \omega^{g-1-i-k}\right]+\sum_{i \geq \max (1, k)}(-1)^{k}\left(\begin{array}{c}
g-k \\
n-i-k
\end{array}\right) \lambda_{k} \Gamma\left[\omega^{g-2-k}\right] \\
& \left.-\frac{1}{2} \sum_{i \geq 1, s, j}(-1)^{g-i-1} \delta_{s *}\left(\psi_{x}^{i-j-3} \psi_{y}^{j}\right)\right) \lambda_{g-i-1} \\
= & \sum(-1)^{k} \lambda_{k}\left[\omega^{i}, \omega^{g-1-i-k}\right]+\left(2^{g-1}-1\right) \Gamma\left[\omega^{g-2}\right]-\sum_{k=1}^{g-1}(-1)^{k} 2^{g-1-k} \lambda_{k} \Gamma\left[\omega^{g-2-k}\right] \\
& \left.-\frac{1}{2} \sum_{i \geq 1, s, j}(-1)^{g-i-1} \delta_{s *}\left(\psi_{x}^{i-j-3} \psi_{y}^{j}\right)\right) \lambda_{g-i-1}
\end{aligned}
$$

Multiplying by $\omega_{1}$ and projecting to $B$ we obtain (compare $[14, \S 7]$ ):

$$
2(2 g-2) m_{2}=\sum(-1)^{k} \lambda_{k} \kappa_{i-1} \kappa_{g-2-i-k}+\left(2^{g-1}-1\right) \kappa_{g-2}+\sum_{k=1}^{g-1}(-1)^{k} 2^{g-1-k} \lambda_{k} \kappa_{g-2-k}
$$

where we set $\kappa_{0}=2 g-2$ for simplicity. This formula is correct over the locus $\mathfrak{M}_{g} \cup \Delta_{0}^{0}$ of curves with at most 1 nonseparating node, but breaks down over the curves with a separating node or a separating pair of nodes, because the naive notion of canonical curve in $\mathbb{P}^{g-1}$ is ill-behaved and requires substantial modification. See [16] for some work in this direction.

4.5. Hyperelliptic locus in genus 3. Our purpose here is to compute the fundamental class of the closure $\mathcal{H E}$ of the locus of smooth hyperelliptic curves of genus 3 in the stable moduli $\overline{\mathcal{M}}_{3}$. Consider a big family $X / B$ parametrized by the locally closed subscheme of a suitable Hilbert scheme of curves in some $\mathbb{P}^{N}$ corresponding to stable curves. $B$ has the two boundary divisors $\delta_{0}, \delta_{1}$, and we have

$$
X_{\delta_{1}}=X_{1} \bigcup_{p_{1} \leftrightarrow p_{2}} X_{2}
$$

with each $X_{i}$ of genus $i$. Let $L=\omega_{X / B}\left(-X_{1}\right)$ where $X_{1} / \delta_{1}$ is the obvious divisor on $X$ (of relative genus 1 ), and let $\mathbb{E}_{1}=\pi_{*}(L)$. The fibre of $\mathbb{E}_{1}$ over a curve in $\delta_{1}$ corresponds to the complete linear system that is the hyperelliptic system on the genus 2 part and the pencil $\left|2 p_{1}\right|$ on the genus- 1 part. Then we have an exact sequence

$$
0 \rightarrow \mathbb{E}_{1} \rightarrow \mathbb{E} \rightarrow \pi_{*}\left(\omega_{X_{1} / \delta_{1}} \otimes \mathcal{O}_{p_{1}}\right) \rightarrow 0
$$

hence $c\left(\mathbb{E}_{1}\right)=c(\mathbb{E})\left(1-\delta_{1}\right)$ and in particular

$$
c_{1}\left(\mathbb{E}_{1}\right)=\lambda_{1}-\delta_{1} .
$$

Also, it is easy to see that

$$
\pi_{*}\left(L^{2}\right)=\pi_{*}\left(\omega^{2}\right)-3 \delta_{1}=\kappa_{1}-3 \delta_{1}, \pi_{*}(L . \omega)=\kappa_{1}-\delta_{1} .
$$

Now consider the rank-1 locus of the natural map over $W^{2}(X / B)$ :

$$
\mathbb{E}_{1} \rightarrow \Lambda_{2}(L)
$$


By Porteous, the fundamental class of this locus in $W^{2}(X / B)$ is computed by

$$
\begin{aligned}
\Delta & =\left\{\frac{c\left(\mathbb{E}_{1}\right)}{c\left(\Lambda_{2}(L)\right)}\right\}_{2} \\
& =-\left(\lambda_{1}-\delta_{1}\right)\left(L_{1}+L_{2}-\Gamma^{(2)}\right)+L_{1}^{2}+\left(L_{2}-\Gamma^{(2)}\right)^{2}+L_{1}\left(L_{2}-\Gamma^{(2)}\right) .
\end{aligned}
$$

Because this locus maps to $B$ with 1-dimensional fibres, we multiply this by $L_{1}$ and then project to $B$. Using Mumford's relation $\kappa=12 \lambda_{1}-\delta$, we compute easily that

$$
\pi_{*}\left(L_{1} \Delta\right)=36 \lambda_{1}-4 \delta_{0}-6 \delta_{1} .
$$

Now the locus $L_{1} \Delta$ clearly covers the hyperelliptic locus 4 times. Additionally, there are three loci over $\delta_{1}$ :

(A) the hyperelliptic pencil on $X_{2}$;

(B) the pencil $\left|2 p_{1}\right|$ on $X_{1}$;

(C) $X_{1} \times p_{2}^{\prime}$ where $p_{2}^{\prime}$ is the image of $p_{2}$ under the hyperelliptic involution.

Each of these, intersected with $L_{1}$, maps to $\delta_{1}$ with degree 2, for a total of 6 . Therefore

$$
[\mathcal{H E}]=\frac{1}{4}\left(36 \lambda_{1}-4 \delta_{0}-12 \delta_{1}\right)=9 \lambda_{1}-\delta_{0}-3 \delta_{1}
$$

a formula first obtained by Harris and Mumford [8]. In higher genus, extra components of excessive dimension appear, requiring substantial modifications (see [16]).

\section{REFERENCES}

[1] M. Aigner, A course in enumeration, GTM, Springer, 2007.

[2] E. Arbarello, M. Cornalba, P. Griffiths, And J. Harris, Geometry of algebraic curves, Springer.

[3] E. CotTerIL, Effective divisors on $\overline{\mathcal{M}}_{\mathrm{g}}$ associated to curves with exceptional secant planes, http://arXiv.org: 1004.0327.

[4] - Geometry of curves with exceptional secant planes, Math. Z. 267 (2011), pp. 549-582.

[5] E. Cotterill, Harvard dissertation (2007) and personal communication.

[6] W. Fulton, Intersection theory, Springer.

[7] I. P. Goulden And D. M. JACKSOn, Combinatorial enumeration, Wiley.

[8] J. Harris And D. Mumford, On the Kodaira dimension of the moduli space of curves, Invent. math. (1982), pp. 23-86.

[9] M. Kontsevich, Intersection theory on the moduli space of curves and the matrix Airy function, Comm. Math. Phys., 147 (1992), pp. 1-23.

[10] M. LeHn, Chern classes of tautological sheaves on Hilbert schemes of points on surfaces, Invent. Math., 136 (1999), pp. 157-207.

[11] Lecture on hilbert schemes, (2004), CRM notes, Montreal.

[12] Gwoнo Liu, The macnodal package for intersection theory on hilbert schemes of nodal curves, web interface (small jobs only) at

http://gwoho.com/macnodal/index.html.a?format=html; source + executable + instructions at http://math.ucr.edu/z̃iv/.

[13] I. G. MacDonald, Symmetric products of an algebraic curve, Topology, 1 (1962), pp. 319-343.

[14] D. Mumford, Towards an enumerative geometry of the moduli space of curves, Arithmetic and Geometry, part II (M. Artin and J. Tate, eds.), Birkhauser, Boston, 1983, pp. 335-368.

[15] H. NAKAJima, Lectures on Hilbert schemes of points on surfaces, University lecture series, Amer. Math. Soc., 1999.

[16] Z. RAN, Boundary modifications of Hodge bundles and enumerative geometry, http://arxiv.org/1011.0406.

[17] _ Intersection theory theory on hilbert schemes of nodal curves: explicit formulas, in preparation.

[18] _ Rational curves in projective spaces, notes available at http://math.ucr.edu/z̃iv/papers/ratcurv.pdf. 
[19] - Structure of the cycle map for Hilbert schemes of families of nodal curves, pp. 1-34, http://arXiv.org/0903.3693.

[20] _ . The degree of the divisor of jumping rational curves, Quart. J. Math., 52 (2001), pp. 367-383.

[21] Cycle map on Hilbert schemes of nodal curve, Projective varieties with unexpected properties (Chiantini et al., ed.), De Gruyter, Berlin, 2005, pp. 363-380.

[22] _ Geometry on nodal curves, Compositio Math., 141 (2005), pp. 1191-1212.

[23] $\longrightarrow$ A note on Hilbert schemes of nodal curves, J. Algebra, 292 (2005), pp. 429-446.

[24] J. G. Semple and L. Roth, Introduction to algebraic geometry, Oxford University Press, 1949.

$[25]$ R. Stanley, Enumerative combinatorics, CUP. 
